\title{
Same Sign W Pair Production in Double Parton Interactions
}




\section{UNIVERSITY OF TWENTE.}

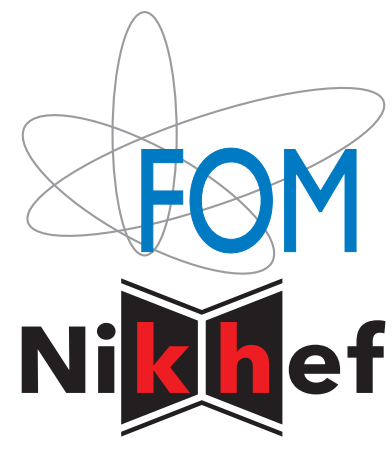

Cover design: Jelena Petrovic ISBN: 978-94-028-0500-0
This work is part of the research program of the "Stichting voor Fundamenteel Onderzoek der Materie (FOM)", which is financially supported by the "Nederlandse organisatie voor Wetenschappelijk Onderzoek (NWO)".

The author was financially supported by the Dutch National Institute for Subatomic Physics (Nikhef). 


\title{
Same Sign W Pair Production in Double Parton Interactions
}

\author{
PROEFSCHRIFT
}

ter verkrijging van

de graad van doctor aan de Universiteit Twente op gezag van de rector magnificus, prof. dr. T.T.M. Palstra, volgens besluit van het College voor Promoties in het openbaar te verdedigen op woensdag 11 januari 2017 om 16.45 uur.

door

Laurentius Jacobus Beemster

Geboren op 11 JULi 1983

TE WAARLAND 


\section{Promotiecommissie:}

Voorzitter: prof. dr. J.W.M. Hilgenkamp Universiteit Twente

Promotor: prof. dr. ing. B. van Eijk Universiteit Twente

Leden: $\quad$ prof. dr. ir. H.H.J. ten Kate prof. dr. P. Mulders

Universiteit Twente prof. dr. M. Merk

VU Amsterdam dr. T. Kasemets

VU Amsterdam prof. dr. A. Pellegrino

Nikhef prof. dr. E. Koffeman

RUG Groningen

UvA Amsterdam 
Dit proefschrift is goedgekeurd door de promotor prof. dr. ing. B. van Eijk. Copyright Lars Beemster 2017. 


\section{CONTEnTS}

Preface

1 Double Parton Interactions $\quad \mathbf{1 0}$

1.1 Double parton scattering cross section . . . . . . . . . . . . 11

1.2 Independent $\mathrm{W}$ pair production hypothesis . . . . . . . . . . . . 13

1.3 Quark interdependence in $\mathrm{W}$ pair production . . . . . . . . . . . 16

2 The LHC accelerator and ATLAS detector 20

2.1 Colliding protons with the LHC . . . . . . . . . . . . . . 20

2.2 Studying collisions with the ATLAS detector . . . . . . . . . . 22

2.2.1 The ATLAS coordinate system . . . . . . . . . . . . 23

2.2.2 Event selection: The ATLAS trigger system . . . . . . . . . 23

2.2.3 Event reconstruction: ATLAS performance . . . . . . . . 29

2.2 .4 Noise reduction in the jet trigger . . . . . . . . . . . 36

3 Data preparation for $\mathrm{W}$ boson pair production in DPI events $\mathbf{5 1}$

3.1 Simulation . . . . . . . . . . . . . . . . 52

3.1.1 DPI simulation using Pythia . . . . . . . . . . . . 52

3.1.2 DPI simulation using Herwig $++\ldots \ldots . \ldots . . . . . .53$

3.1.3 Comparison between Pythia and Herwig ++ . . . . . . . . . 54

3.2 Signal: Event topology . . . . . . . . . . . . . . . . 56

3.3 Noise: Processes with similar topology . . . . . . . . . . . . . . 59

3.3.1 Single muon + fake muon final state: $W+$ jets . . . . . . 59 
3.3.2 Three muon final state: $\mathrm{W}+\gamma^{*} / \mathrm{Z} \ldots \ldots . \ldots . \ldots 61$

3.3.3 Four muon final state: ZZ . . . . . . . . . . . . . . . 62

3.4 Dataset preparation . . . . . . . . . . . . . . 62

3.4.1 Cuts on muon momentum . . . . . . . . . . . . 63

3.4.2 Cuts on missing transverse momentum . . . . . . . . . . 64

3.4.3 Cuts on $\mathrm{W}$ boson reconstruction variables . . . . . . . . . 65

3.4.4 Additional cuts on data quality . . . . . . . . . . . . . 65

3.5 Data analysis method . . . . . . . . . . . . . 66

3.5.1 Signal/background separation with Neural Networks . . . . 67

3.5.2 Data fitting procedures . . . . . . . . . . . . . . . 69

3.5.3 Summary . . . . . . . . . . . . . . . 71

4 Analysis of W boson pair production in DPI events 72

4.1 Contamination of the signal data set . . . . . . . . . . 73

4.1.1 Fake background class validation . . . . . . . . . . . . . 74

4.2 Null hypothesis and signal hypotheses . . . . . . . . . . . . . . 77

4.3 The DPI W pair production cross section . . . . . . . . . . . 80

4.4 Conclusion . . . . . . . . . . . . . . . . 82

5 Conclusion and prospects $\quad \mathbf{8 4}$

5.1 Interpretation of results . . . . . . . . . . . . . . . . 84

5.2 Prospects and recommendations for further research . . . . . . . . 87

$\begin{array}{lr}\text { Bibliography } & \mathbf{9 0}\end{array}$

$\begin{array}{ll}\text { Summary } & 95\end{array}$

$\begin{array}{lr}\text { Samenvatting } & 102\end{array}$

$\begin{array}{ll}\text { Acknowledgements } & 109\end{array}$ 


\section{PREFACE}

The beginning of the 21st century is an extremely exciting time for High Energy Physics. The world's most powerful particle accelerator, the Large Hadron Collider (LHC), is fully operational and colliding protons at unprecedented energies. The associated detector collaborations made a sensational breakthrough discovering the Higgs boson in the data collected during 2012. [1]

In this high energy environment present in the LHC's collisions even more new particles might be waiting to be discovered. Together with new physics, already known objects can be studied in new ways and in greater detail. One such new research opportunity that is available in the LHC is formed by Multiple Parton Interactions (MPI), which will be shown in this document to be a promising new probe of the proton structure.

Together with this physics potential, MPI also represents a major signal contamination for other new physics studies. Due to the high energies at which those previously unseen physics processes might reveal themselves, it is likely they produce multiple bosons in the final state. MPI also has a strong ability to produce two or more heavy bosons in a single event, making it a significant background process to this multiple boson new physics. A prime example is the aforementioned Higgs boson. With the currently available data sample only its basic attributes can be determined: mass, spin, parity and a set of decay modes.

The measured values for these parameters are matching those predicted for the Higgs boson by the Standard Model. This theory assumes more properties, in particular its so called "Mexican Hat" potential given in equation 1 [2] and shown in figure 1.

$$
V(\Phi)=m^{2} \Phi^{\dagger} \Phi+\lambda\left(\Phi^{\dagger} \Phi\right)^{2}
$$

The mass term in the potential $\left(\Phi^{\dagger} \Phi\right)$ can be quantified from processes such as shown in figure 2, and the remaining variable $\lambda$ defines the magnitude of the Higgs self-coupling $\left(\Phi^{\dagger} \Phi\right)^{2}$. An example of this self-coupling process is given in figure 3 . 


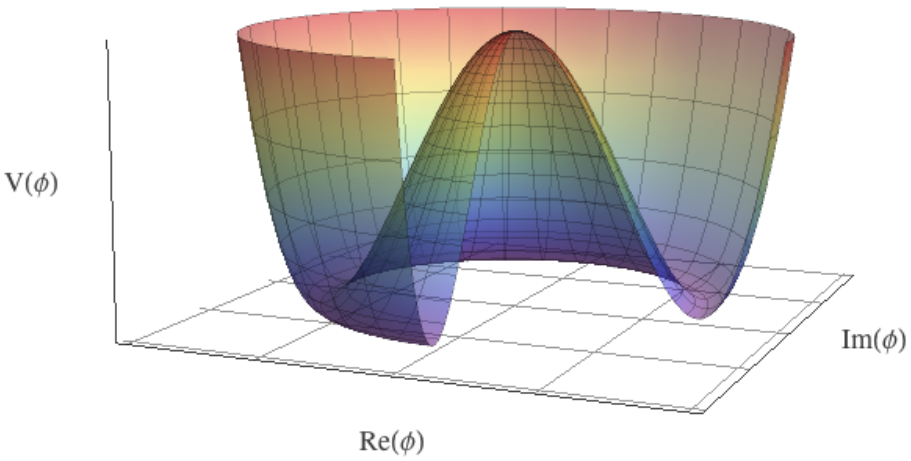

Figure 1: The Higgs potential in the Standard Model. The field has a non-zero amplitude at the ground state due to the "bump" at the origin. This unique property of the Standard Model Higgs boson is yet to be verified by experimental data. The data set necessary to study this structure will be contaminated with MPI events.

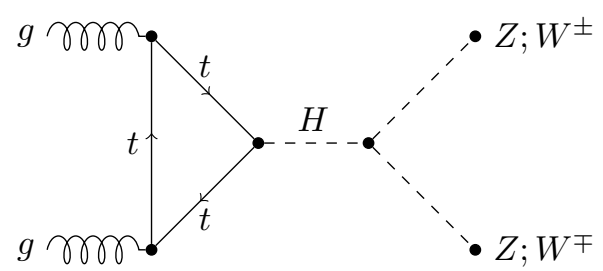

Figure 2: Higgs boson production via top quark loop and two examples from the set of decay modes. Both these decay modes contain two heavy bosons in the final state, which can also be produced in MPI interactions. Those type of events are used to measure the mass of the Higgs boson by studying the energy and momenta of the outgoing particles.

To measure the shape of the potential of the recently discovered particle, the Higgs mass and also the Higgs self-couplings have to be studied. In other words, events with two Higgs bosons in the final state have to be selected from the event sample produced by the collider. This is an example where (multiple boson) MPI events will enter the signal (two Higgs bosons) data set as a contamination. It is therefore paramount to fully understand MPI, they are a prime background for new physics processes. The total production cross sections $\sigma_{t o t}$ for the mentioned processes involving the Higgs particle are given in table 1, as well as an MPI process that will contaminate the data set. 


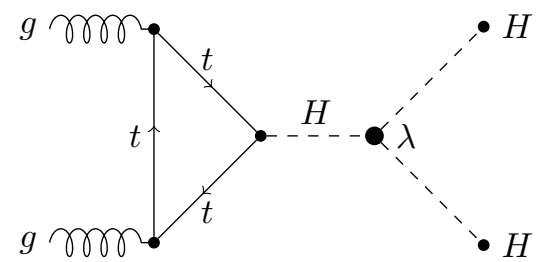

Figure 3: Higgs boson production via top quark loop and decay via the Higgs selfcoupling. This self-coupling gives a handle on the $\lambda$ parameter in the potential given in equation 1, and together with the mass provides a full test of the Standard Model Higgs potential.

\begin{tabular}{|c|cc|}
\hline Process & $\sigma_{\text {tot }}(\mathrm{fb})$ & \\
\hline$p p \rightarrow H \rightarrow W^{+} W^{-}$ & $4.8 \times 10^{3}$ & {$[2]$} \\
$M P I \rightarrow W^{+} W^{-}$ & 150 & {$[3]$} \\
$p p \rightarrow H \rightarrow H H$ & 9 & {$[4]$} \\
\hline
\end{tabular}

Table 1: Production cross sections relevant to the motivation for this analysis. The MPI process is actually two hard scatters, and thus is usually called a Double Parton Interaction (DPI) process. The cross section $\sigma$ is linearly related to the number of events in the data set. The cross section for the first process is about 30 times larger than the MPI process on the second row, so for every $W^{+} W^{-}$ pair from MPI there are 30 such pairs in the data set from Higgs decay. The third row is a new physics process which will have DPI processes as background in future analyses.

The first and second cross sections in table 1 indicate the dominance of single Higgs processes over DPI processes in multiple heavy boson final states. This relation is inverted when considering double Higgs production (the third row of table 1), which is relevant in studying the Higgs potential. This means that in the currently available data set, in which the Higgs boson was discovered, finding DPI processes with multiple heavy boson final states will be a challenge. On the other hand, in future studies of the Higgs potential, those same DPI processes are going to be a prime background. This is due to the importance of the heavy boson decay channels in Higgs boson analyses, see for example the Higgs discovery analysis [1]. 


\section{Proton composition}

The characteristics of MPI events are in case of proton-proton collisions completely determined by the structure of the proton. Since the 1970s the proton has been known to be composed of partons, which are the quarks and gluons proposed by Zweig [5] and Gell-Mann [6] in 1964. The interactions between quarks and gluons are described in the Quantum Chromodynamics (QCD) framework which was founded on the work of Gross, Wilczek and Politzer [7,8]. Quarks have the ability to form composite particles called hadrons. Hadrons formed by a quark-antiquark pair are characterized as mesons, and combinations with three (anti)quarks are labeled baryons. Two prime examples of baryons are protons and neutrons. Gluons mediate the binding force to keep the partons together.

The model of the proton consisting of three quasi free non-interacting quarks close together is too crude. Those three quarks are called the valence quarks, and they determine the quantum numbers of the proton. The valence quarks are immersed in a sea of virtual quark-antiquark pairs created by the gluons keeping the valence quarks together. One key element of QCD are the Parton Distribution Functions (PDFs). They represent the probability densities to find any parton carrying a momentum fraction $x$ of the proton longitudinal momentum, given the momentum transfer $Q^{2}$ in the interaction. Two examples of a PDF at different values of $Q^{2}$ are given in figure 4 . It is important to note that the PDF is a feature of an interaction, and not of a free proton. In this sense it can be considered a snapshot of the parton densities at time of collision, the precise constituents of a free proton are indeterminate due to their quantum nature.
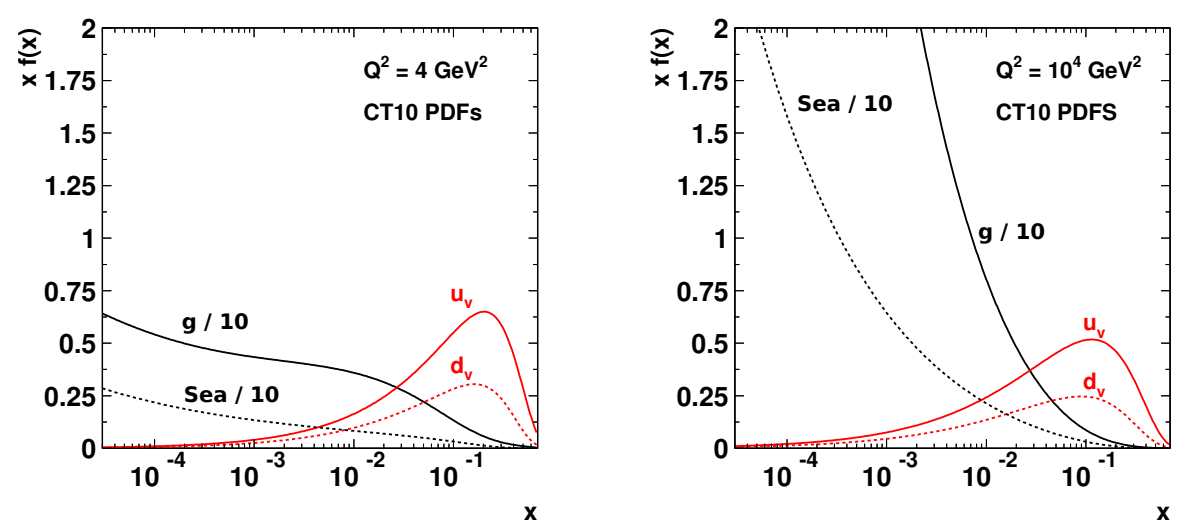

Figure 4: Example PDFs at $Q^{2}=4 \mathrm{GeV}^{2}$ and $Q^{2}=10^{4} \mathrm{GeV}^{2}$ : the scaled gluon density (g), the scaled sea quark densities ('Sea') and the valence up $\left(\mathrm{u}_{v}\right)$ and down $\left(\mathrm{d}_{v}\right)$ quark densities. Taken from $[9]$. 
This current proton model based on the PDFs is very rudimentary. It treats the partons as independent entities inside the proton, and only takes into account their momentum. The proton in reality is highly complex due to the quantum numbers the quarks and gluons carry, and the partons are certainly not independent of each other. They all carry spin that add to the total spin of the proton. They also have a quantum number called color charge. This important property gives the name to the theory of Quantum Chromodynamics. The combined color of all partons has to cancel out, since the proton is color neutral. These two arguments definitively provide a correlation between the partons, as changing the attributes of one parton has to induce a reaction from another parton to keep the proton stable. A second argument of the crudeness of the model is the acquisition of the PDFs itself. These functions can only be obtained from experimental data, and can not be derived from first principles. In experiments the proton structure is probed in scattering processes like Deep Inelastic Scattering (DIS), in which for example electrons are used as a probe to transfer a four momentum to the proton in a collision.

A significant limitation of the current PDFs is that they only give the probabilities to find a single parton of a certain flavor in the proton with a given momentum. It does not give any information about the probability of finding multiple partons at once, or whether or not the partons are independent. This is of particular importance when multiple partons from the same proton simultaneously have a hard interaction with partons from a second proton.

In MPI events multiple partons from the same colliding protons are contributing to the overall interaction. A subset of these MPI events is where two partons from one proton are interacting with two partons from the other in two separable processes. These are called Double Parton Interactions (DPI) and they are the main subject of this thesis.

\section{Current experimental status and opportunities}

A theoretical framework for DPI exists, where the interaction cross section is fully factorized into the product of the constituent cross sections. There is an ongoing effort to model DPI using separate PDFs for individual partons, with a correlation between the different PDFs in the event. These theories are discussed in chapter 1 and require experimental confirmation.

Until now, the experimental evidence for DPI is solely based on processes with jets in the final state, meaning that the underlying process is not strictly defined since jets are produced in a large variety of interactions. This uncertainty in production mechanism makes those past experiments insensitive to variations in event rate between different initial states. The most precise measurements on DPI are performed at the Tevatron collider, in both the D0 [10] and CDF [11] 
experiments. The main aim of these DPI analyses was to determine the event rate of DPI events, and lacked the statistics to do a topological study on the underlying processes. The results of these studies are summarized in section 1.2.

The LHC opens up the possibility of studying a DPI process with a clear production mechanism, namely $\mathrm{W}$ pair production. W pair production from DPI events is studied in the 2012 data set produced by the ATLAS experiment and the method and results are summarized in this document. A theoretical treatment is used to determine variables of interest, and show that the angular distributions of the decay products of the $\mathrm{W}$ bosons give valuable information about the proton structure. These particular details were previously inaccessible. The focus of the study is to determine if there is sufficient data taken to prove the existence of this production process. And if so, is there sufficient data to assess correlations between partons in the colliding protons?

In the high energy, high event rate environment of the LHC a typical event already has multiple primary proton-proton interactions as shown in figure 5 . This pile-up of processes is a major concern in this study, because when two

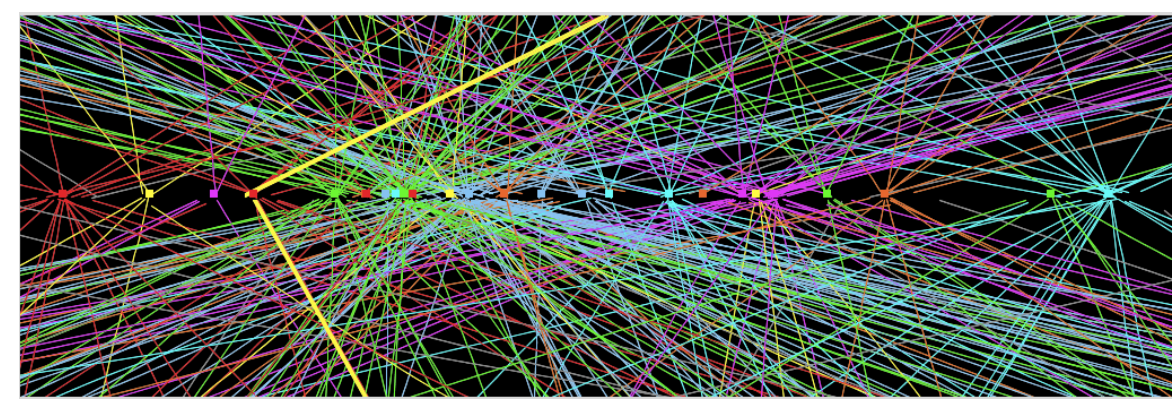

Figure 5: Multiple vertices reconstructed in a candidate $\mathrm{Z}$ boson event. The figure is a side view of the center of the detector. In this particular event a proton collision occurred at every origin, 25 in total. The full ATLAS 2012 data set consists of multiple billion of such events.

'normal' processes overlap they behave exactly like a single DPI process where the partons are uncorrelated. This issue is addressed in sections 2.2 and in the discussion of the ATLAS inner detector in section 2.2.2.

The correlation between two individual interacting quarks from a single proton in a DPI event is not experimentally verified yet. Therefore I tested two hypotheses. The first hypothesis predicts a fully factorized relation, indicating that there is no (measurable) relationship between the two quarks in a single proton that take part in the DPI process. The second, and much more interesting hypothesis, foresees a connection between the two quarks. 
In QCD the coupling constant $\alpha_{s}$ is a function of the mass of the produced particle, or the energy scale at which the interaction occurs. When the two quarks are interdependent, it also suggests that the energy scale at which either interaction takes place may be correlated. In case the second hypothesis is proven, the coupling constant in DPI events is possibly affected too.

This possible dependence can present itself in the data in a variety of ways: final state energy, angle of decay, overall production cross section and any combination of these variables. The hypothesis anticipating a factorized relation is taken as a starting point, because first the DPI W pair production process itself has to be proven to exist.

The motivation for this analysis is two-fold. We want to study previously inaccessible signatures suggesting correlations between partons. We use a novel method relating the angular properties of the final state with the polarization of the initial state. This connection is schematically depicted in figure 6 and the supporting theory is presented in section 1.3.
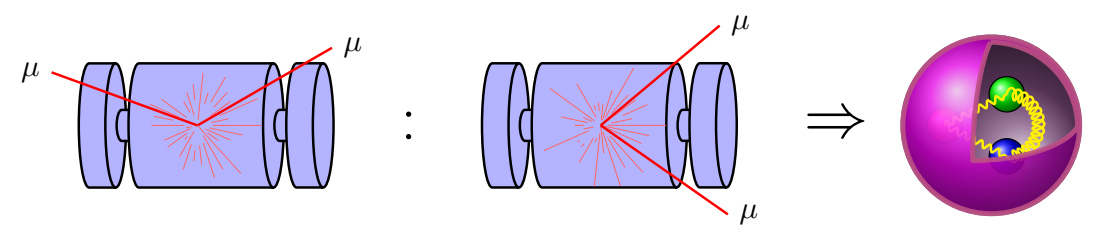

Figure 6: The ratio of DPI events where the final state muons from two semileptonically decaying $\mathrm{W}$ bosons traverse opposite hemispheres compared to events where they pass through the same hemisphere is related to the polarization of the initial state. This opens a window revealing the inner structure of the proton.

Second, we want to determine if multi boson DPI is a significant background process in new physics studies, which is a realistic possibility taking table 1 into account. This places the analysis on the very edge of the possibilities provided by the current size of the available data set. Both bases indicate the particular processes are not experimentally reviewed yet, which means the possibility exists that no signal events can be detected at all. This would however still be an important result for the latter motivation, because it will set an upper limit on the event rate. New physics studies on processes with an event rate much higher than this upper limit will thus not be affected by this background.

The presence of two $\mathrm{W}$ bosons in the signal event is used to try to distinguish it from background processes. In section 3.2 the unique final state features of a DPI W pair production event are discussed. In order to reconstruct the $\mathrm{W}$ bosons an additional selection on the properties of the outgoing particles is applied. In our analysis we try to define a data set consisting of only $\mathrm{W}$ boson pairs with 
equal sign, both decaying into a muon and muon neutrino. Muons are chosen because they provide a far superior detection efficiency compared to other leptons, especially considering charge identification. The selection on equal charge is made because the ability of DPI to create only two isolated muons of same sign in the final state (and nothing else) is extremely rare in the Standard Model. This selection limits the statistics for this analysis even further. Combined with the facts that W boson in DPI only constitutes a small background in other studies and the ATLAS 2012 data set is the first with significant events that $\mathrm{W}$ boson pair studies might be possible, just proving the existence of this DPI process with two same sign $\mathrm{W}$ bosons decaying to muons will be a daunting task.

The signal in this analysis is extremely small, so an important aspect of the procedure is to minimize the signal to noise ratio. The full ATLAS 2012 data set consists of 250 billion inelastic proton-proton collisions. The detector itself cuts this by several orders of magnitude by filtering out low energy events. The remainder of the events are written to disk and filtered off-line. In our analysis this detector data set is cleaned until there are only 750 potential signal events left. Using the W-pair cross section from table 1 and the branching fraction of the $W^{ \pm} W^{ \pm} \rightarrow \mu^{ \pm} \mu^{ \pm}$of around 1\% [2] the expected signal contribution in this data sets can be as high as 30 events. Section 2.1 explains how to calculate this approximation. A more detailed simulation of the signal process in chapter 3 however indicates an event yield an order of magnitude lower.

The bulk of these events is expected to be from other processes than the DPI signal process. In this signal data set the main background processes, $W+$ jets and $W+\gamma^{*} / Z$, are analyzed first to determine their relative contribution. To test if there is an additional $\mathrm{W}$ pair from DPI a number of simulated data sets is generated with the estimated background contributions. The sole difference between each simulated data set is that every subsequent set contains a larger signal contribution. These simulated data sets are then compared to the data set obtained from the experiment using Neural Networks, and the signal contribution of the most similar one is taken as the final analysis prediction. The result of this analysis is an estimate of the cross section of same sign $\mathrm{W}$ pair production in DPI events. Variables sensitive to the proton structure in these events are identified and the prospects for future research on this subject are given to conclude the study. 


\section{Outline}

In chapter 1 I explain the theoretical framework to study Double Parton Interactions. In this context I focus on the $\mathrm{W}$ pair production process and its expected event rate predicted by theory. I explain the two hypotheses that I tested: the proposition of completely independent partons and the assumption that the quarks in the proton-proton collision are correlated. For the latter concept I explain the observables that will give an indication to this interdependence.

Chapter 2 is a brief description of the experimental environment. The technical details of the LHC accelerator are given. The ATLAS particle detector is dissected and subsystems described with emphasis on the sections relevant for muon detection. For every physics object used in my analysis I give the detection precision and efficiency achieved with ATLAS. I use the chronological data flow as seen by the trigger subsystem as a guideline.

In chapter 3 I describe my analysis method. A description of the Monte Carlo event generators is given, and I discuss the event topology of the W pairs produced in DPI events. Processes with similar topologies that contaminate my data set are introduced and illustrated here. Using the distributions obtained from the simulations of the signal and background data sets I discuss the cuts on the variables and the preparation of the data sets used in my study. A treatment of the multivariate techniques I used in my research is added at the end of this chapter.

Chapter 4 is an account of the results obtained. It starts with the verification of the extent of signal contamination in the data set. I further discuss the outcome of the two hypotheses which spurred my research, after which I draw conclusions concerning the event rate of same sign W pair production in DPI process.

A concluding chapter 5 contains an interpretation of all results and the prospects and recommendations for future research in this area. 


\section{DOUBle PARTON INTERACTIONS}

Particle accelerators are a particle physicist's prime tool to study the fundamental dynamics of nature. When colliding elementary particles like electrons, the initial state (all the incoming particle attributes like energy, flavor, spin, charge) of the process is well defined. In proton collisions this is not the case. Protons consist of multiple partons, quarks and gluons, with varying attributes. For most studies these collisions are modeled as one parton from each proton interacting, with the specific parton type as an unknown with probabilities determined by the collision's Parton Density Function (PDF). For example the Higgs boson discovered recently has its production processes modeled this way.

Sometimes two partons from a proton interact simultaneously with two partons from the other proton. These events are called Double Parton Interactions (DPI). When both these hard interactions produce a $\mathrm{W}$ boson it is possible to infer additional information about the initial state. Furthermore, are the $\mathrm{W}$ bosons produced independently, or are they part of a larger coherence? To answer this question, two hypotheses are tested. The hypothesis of independent $\mathrm{W}$ production assumes that the two bosons are produced as they would in the single W production process. The coherent $\mathrm{W}$ pair production hypothesis is a lot more interesting as described in section 1.3. Since the $\mathrm{W}$ only couples to left handed quarks, the presence of longitudinal spin correlations between the quarks in the DPI process changes the $\mathrm{W}$ pair production cross section. More importantly, it changes the rapidity distributions of the final state leptons when both $\mathrm{W}$ bosons decay semi-leptonically [12]. 


\subsection{Double parton scattering cross section}

To determine if two hard scatter double parton interactions are correlated or not, we first need to understand how a single interaction behaves. When two protons collide, or move through each other, the majority of the partons inside the protons do not notice the presence of the partons from the other proton. When two partons do interact, the leading order scattering cross section $\sigma$ can be factorized into a short distance, high energy part and a long distance part:

$$
\sigma=\sum_{i j} \hat{\sigma}_{i j} \iint f_{i}(x) f_{j}(\bar{x}) d x d \bar{x}
$$

Two partons $i$ and $j$ interact with a partonic cross section $\hat{\sigma}_{i j}$ forming the high energy, short distance part of the cross section. The long distance part is given by the parton density functions $f_{i}\left(f_{j}\right)$, describing the probability of finding parton $i(j)$ with a fraction $x(\bar{x})$ of the longitudinal momentum of the proton.

The rapidity distributions of leptons generated by W boson decay in DPI events are sensitive to the polarization of the initial state quarks (see section 1.3 ), so it is important to know the angular dependence of the partonic cross section, reproduced from [13]:

$$
\frac{d \hat{\sigma}}{d \Omega}=\left(1+\cos ^{2} \theta\right) K_{a \bar{a}}(Q) \pm 2 \cos \theta K_{a \bar{a}}(Q)
$$

with $a=q, \Delta q, \bar{a}=\bar{q}, \Delta \bar{q}$ where $q$ is an unpolarized quark and $\Delta q$ a longitudinally polarized quark, and integration element $d \Omega=d \phi d \cos \theta$.

The coupling factor $K$ is given in [12] as

$$
K_{q_{i} \bar{q}_{j}}=\frac{\alpha^{2}}{4 N_{c}} \frac{\left|V_{q_{i} q_{j}}\right|^{2}}{\left(2 \sin \theta_{w}\right)^{4}} \frac{Q^{2}}{\left(Q^{2}-m_{W}^{2}\right)^{2}+m_{W}^{2} \Gamma_{W}^{2}}
$$

The angle $\theta$ is the polar angle between the $z$ axis formed by the incoming proton momenta and the direction of the outgoing leptons in the $\mathrm{W}$ boson rest frame, as illustrated in figure 1.1.

In a similar way we can write the double parton scattering process as a decomposition of the long and short range physics. The short range part now consists of two partonic cross sections, and the long range part is formed by two functions describing the probability of finding two partons with longitudinal momentum fraction $x_{1}$ and $x_{2}$. However, the long range physics has an additional degree of freedom, the transverse distance $\vec{y}$ between the two interactions. See for example equation 2.37 in [14].

$$
\sigma_{D P I} \propto \sum_{i j k l} \hat{\sigma}_{i j} \hat{\sigma}_{k l} \int d x_{1} d x_{2} \int d \bar{x}_{1} d \bar{x}_{2} \int d^{2} \vec{y} f_{i k}\left(x_{1}, x_{2}, \vec{y}\right) f_{j l}\left(\bar{x}_{1}, \bar{x}_{2}, \vec{y}\right)
$$




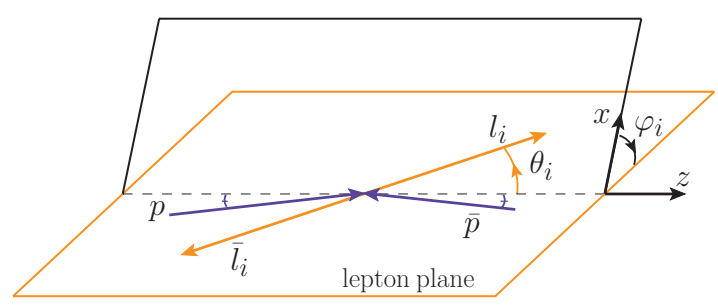

Figure 1.1: The coordinate system used in the cross section equations (1.2 and 1.3). The $z$ axis is formed by the momenta of the incoming protons ( $p$ and $\bar{p})$, the produced $\mathrm{W}$ boson is at rest. In this boson rest frame the final state leptons $\left(l_{i}\right.$ and $\left.\bar{l}_{i}\right)$ are produced anti-parallel. Figure taken from [12].

The sum in equation 1.4 runs over both flavor and polarization. We now decompose the double parton distributions (DPDs) $f_{p q}\left(x_{1}, x_{2}, \vec{y}\right)$ in different combinations of quark polarizations following [12]. For unpolarized quarks we use

$$
f_{p q}\left(x_{1}, x_{2}, \vec{y}\right)=f_{q_{1} q_{2}}
$$

For now this short description should suffice, a more detailed explanation follows shortly in section 1.3.

Inserting the equation of the partonic cross section from 1.2 into 1.4 and thus taking into account the angular dependence we obtain the following expression, as shown by Kasemets [12] but here neglecting the spin correlations:

$$
\begin{aligned}
& \frac{d \sigma^{W W}}{\prod_{i=1}^{2} d x_{i} d \bar{x}_{i} d \Omega_{i}}=\frac{1}{C} \sum_{q_{1} q_{2} q_{3} q_{4}} K_{q_{1} \bar{q}_{3}} K_{q_{2} \bar{q}_{4}} \\
& \times\left\{\left(1+\cos \theta_{1}\right)^{2}\left(1+\cos \theta_{2}\right)^{2} \int d^{2} \vec{y}\left(f_{q_{1} q_{2}} \bar{f}_{\bar{q}_{3} \bar{q}_{4}}\right)\right. \\
& +\left(1+\cos \theta_{1}\right)^{2}\left(1-\cos \theta_{2}\right)^{2} \int d^{2} \vec{y}\left(f_{q_{1} \bar{q}_{4}} \bar{f}_{\bar{q}_{3} q_{2}}\right) \\
& +\left(1-\cos \theta_{1}\right)^{2}\left(1+\cos \theta_{2}\right)^{2} \int d^{2} \vec{y}\left(f_{\bar{q}_{3} q_{2}} \bar{f}_{q_{1} \bar{q}_{4}}\right) \\
& \left.+\left(1-\cos \theta_{1}\right)^{2}\left(1-\cos \theta_{2}\right)^{2} \int d^{2} \vec{y}\left(f_{\bar{q}_{3} \bar{q}_{4}} \bar{f}_{q_{1} q_{2}}\right)\right\} \\
& \quad+\{\text { interference terms }\}
\end{aligned}
$$

where the combinatorial factor $C$ is equal to 2 when the final states of the two interactions are the same, and 1 otherwise. The interference terms are from flavor and fermion number interference, but are not considered in this study and 
averaged over in the analysis. The effect on the rapidity distribution is more clear if this equation is written as:

$$
\frac{d \sigma^{W W}}{\prod_{i=1}^{2} d x_{i} d \bar{x}_{i} d \Omega_{i}}=\frac{1}{C} \sum_{q_{1} q_{2} q_{3} q_{4}} K_{q_{1} \bar{q}_{3}} K_{q_{2} \bar{q}_{4}} \times\left(A \cdot \int d^{2} \vec{y} F\right)
$$

with

$$
A=\left[\begin{array}{c}
\left(1+\cos \theta_{1}\right)^{2}\left(1+\cos \theta_{2}\right)^{2} \\
\left(1+\cos \theta_{1}\right)^{2}\left(1-\cos \theta_{2}\right)^{2} \\
\left(1-\cos \theta_{1}\right)^{2}\left(1+\cos \theta_{2}\right)^{2} \\
\left(1-\cos \theta_{1}\right)^{2}\left(1-\cos \theta_{2}\right)^{2}
\end{array}\right]
$$

and $F$ the collinear double parton distributions:

$$
F=\left[\begin{array}{c}
f_{q_{1} q_{2}} \bar{f}_{\bar{q}_{3} \bar{q}_{4}} \\
f_{q_{1} \bar{q}_{4}} \bar{f}_{\bar{q}_{3} q_{2}} \\
f_{\bar{q}_{3} q_{2}} \bar{f}_{q_{1}} \bar{q}_{4} \\
f_{\bar{q}_{3} \bar{q}_{4}} \bar{f}_{q_{1} q_{2}}
\end{array}\right]
$$

This relation between $A$ and $F$ shows that the rapidity distributions of the final state are related to the parton density functions. Specifically, the rate of events where the muons are detected in the same or opposite hemispheres of the detector is sensitive to the polarization of the quarks inside the proton. This is further explained in section 1.3.

In the next section the DPI cross section is factorized into the product of two single parton cross sections:

$$
\sigma_{D P I}^{(A, B)} \propto \sigma^{A} \sigma^{B}
$$

In addition to the DPI production process the final state $(\mathrm{A}, \mathrm{B})$ can also be produced in a single hard scattering event with cross section $\sigma_{S}^{A B}$. When the final state masses are fixed, the center of mass energy $\sqrt{s}$ dependence of the cross section is directly related to the $x$ dependence in the PDFs. Since the PDFs increase rapidly when $x \rightarrow 0$, the cross sections grow with rising $\sqrt{s}$. Since $\sigma_{D P I}^{(A, B)}$ can be considered the product of two hard scatters, it will be boosted more easily than $\sigma_{S}^{A B}$ which is a single hard scatter (see also [3]). This relation makes DPI a possible significant background process to rare new physics events, especially when these events require a large center of mass energy and have a final state $(A, B)$ that can also be produced with two single events with final states $A$ and $B$.

\subsection{Independent $\mathrm{W}$ pair production hypothesis}

The most simple hypothesis for $\mathrm{W}$ pair production in DPI events is where both $\mathrm{W}$ bosons are produced seemingly independent of each other. This means that 
the partons involved in the process are not, or at least not measurably, correlated.

In the independent partons hypothesis the cross section 1.4 can be parametrized by assuming that the transverse distance $\vec{y}$ is independent of the momentum fractions $x$ and $\bar{x}$. With the additional assumption that also the double parton distributions can be separated in two normal (better known) parton distributions $f\left(x_{1}, x_{2}, \vec{y}\right)=f\left(x_{1}\right) f\left(x_{2}\right) g(\vec{y})$, we can write the double parton cross section in terms of single parton cross sections using the following formula:

$$
\frac{d \sigma_{D P I}^{W W}}{\prod_{i=1}^{2} d x_{i} d \bar{x}_{i}}=\frac{d \sigma_{W} d \sigma_{W}}{C \sigma_{e f f}}
$$

where

$$
\frac{1}{\sigma_{e f f}}=\int g(\vec{y}) d^{2} \vec{y}
$$

with $g(\vec{y})$ the separation between the two separate processes, which can be regarded as the transverse area in the collision where the second interaction takes place. This is much like an effective cross section for the second hard interaction, in the same way the inelastic proton-proton cross section appears in the single $\mathrm{W}$ production probability.

$$
\begin{gathered}
\sigma_{p p}=\sum \sigma_{i}, i \in\{2 j, W, Z, b \bar{b}, H, . . .\} \\
\sigma_{e f f}=\sum \sigma_{i}^{2 n d}, i \in\{2 j, W, Z, b \bar{b}, H, . .\}
\end{gathered}
$$

It turns out that a nice ball-park estimate for $\sigma_{e f f}$ is given by the most simplified approximation; The average overlap of the two inelastic proton-proton cross sections is compatible with current measurements [15-19,22]. This is tested by considering the schematic representation of a proton-proton collision in the transverse plane, given in figure 1.2.

The area of overlap of two unit circles is given by:

$$
A=2 \cos ^{-1}\left(\frac{b}{2}\right)-\frac{b}{2} \sqrt{4-b^{2}}
$$

To calculate the average area of overlap we see that a small area is much more likely. The weight function $\omega(b)$ only depends on the impact parameter $b$ :

$$
\omega(b)=2 \pi b
$$

The average is now reduced to a simple integral:

$$
A_{\text {avg }}=\frac{\int_{0}^{2}(A \omega) d b}{\int_{0}^{2}(\omega) d b}=\pi / 4
$$




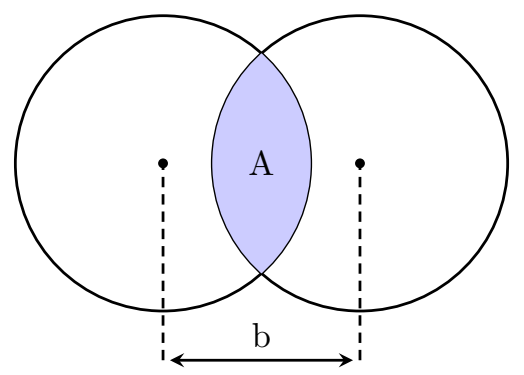

Figure 1.2: The area of overlap $A$ of a proton-proton collision with impact factor $b$ in the transverse plane. It is shown that the current DPI measurements are compatible with $\sigma_{\text {eff }}=A$.

Since the area of a unit circle is equal to $\pi$, the average area of overlap is simply a quarter of the total area. In our case that means $\sigma_{e f f}$ is one quarter of the inelastic proton-proton cross section:

$$
\sigma_{e f f}=\sigma_{p p} / 4
$$

The previous measurements and their relation to $\sigma_{p p} / 4$ of the DPI effective cross section $\sigma_{\text {eff }}$ are given in figure 1.3.

The inelastic proton cross section $\sigma_{p p}^{\text {inel }}$ as measured by the ToTEM collaboration [23] corresponds to the value when the data for this study was taken. They measured a value of $74.7 \pm 1.7 \mathrm{mb}$, which gives $\sigma_{\text {eff }}=18.5 \pm 0.4 \mathrm{mb}$ for the $8 \mathrm{TeV}$ 2012 data. The value commonly suggested from the Tevatron measurements is $\sigma_{\text {eff }} \approx 15 \mathrm{mb}[3]$.

To test the independent production hypothesis the final state with two muons of same sign is chosen. The choice for same sign leptons is motivated by the fact that no proven process other than DPI produces just two (no more, no less) leptons of equal charge, without any significant QCD (jet) energy in the event. The muon channel is selected because of the better detection efficiency compared to the electrons.

In the study Neural Networks are trained on the muon characteristics of the same sign muon events. They are designed to distinguish the true DPI events from the background events caused by detection inefficiencies, as explained in section 3.5.1. These Neural Networks are then tested on sets of simulated data with varying DPI contributions in section 4.2 , after which the responses are compared with the classifier output from the real data set. 


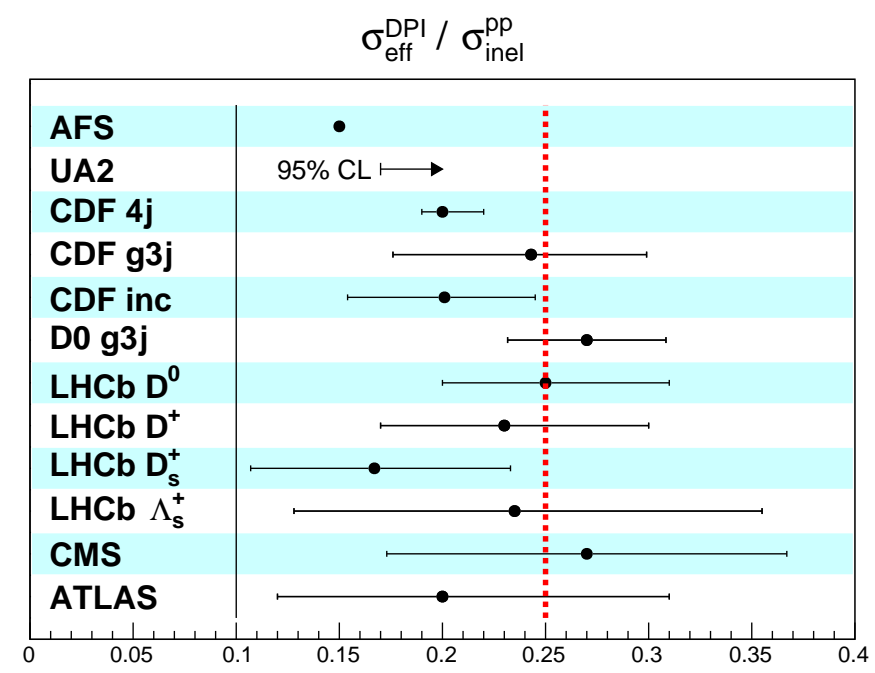

Figure 1.3: Measurements of $\sigma_{\text {eff }}$ and their relation to $\sigma_{i n e l}^{p p}$. The dotted line indicates the naive approximation of $\sigma_{e f f}=\sigma_{\text {inel }}^{p p} / 4$. Data taken from [15-22].

\subsection{Quark interdependence in $\mathrm{W}$ pair production}

A second hypothesis with a more complex structure is based on the assumption that the quarks involved in the $\mathrm{W}$ pair production in each proton have quantum numbers statistically dependent on one another. This will result in a correlation in the characteristics of the two produced $\mathrm{W}$ bosons, and also their decay products.

The hypothesis in our study takes into account the polarization (or helicity) of the quarks. Since W bosons couple only to left-handed fermions, the quarks have to be unpolarized or longitudinally polarized. The transverse polarization state is a superposition of the left-handed and right-handed polarization state, and thus invisible to the $\mathrm{W}$ boson. This simplifies the cross section considerably since the transverse polarization contribution is zero.

The polarization dependence changes the factor $F$ in equation 1.7. Instead of neglecting spin correlations using

$$
F_{q q}=\left[\begin{array}{cc}
f_{q_{1} q_{2}} \bar{f}_{\bar{q}_{3} \bar{q}_{4}} \\
f_{q_{1} \bar{q}_{4}} \bar{f}_{\bar{q}_{3} q_{2}} \\
f_{\bar{q}_{3} q_{2}} \bar{f}_{q_{1} \bar{q}_{4}} \\
f_{\bar{q}_{3} \bar{q}_{4}} \bar{f}_{q_{1} q_{2}}
\end{array}\right]
$$


as in the independent cross section, an expression where the polarization is taken into account is used [12]:

$$
F_{(q q, \Delta q \Delta q)}=\left[\begin{array}{l}
\left(f_{q_{1} q_{2}}+f_{\Delta q_{1} \Delta q_{2}}\right)\left(\bar{f}_{\bar{q}_{3} \bar{q}_{4}}+\bar{f}_{\Delta \bar{q}_{3} \Delta \bar{q}_{4}}\right) \\
\left(f_{q_{1} \bar{q}_{4}}-f_{\Delta q_{1} \Delta \bar{q}_{4}}\right)\left(\bar{f}_{\bar{q}_{3} q_{2}}-\bar{f}_{\Delta \bar{q}_{3} \Delta q_{2}}\right) \\
\left(f_{\bar{q}_{3} q_{2}}-f_{\Delta \bar{q}_{3} \Delta q_{2}}\right)\left(\bar{f}_{q_{1}} \bar{q}_{4}-\bar{f}_{\Delta q_{1} \Delta \bar{q}_{4}}\right) \\
\left(f_{\bar{q}_{3} \bar{q}_{4}}+f_{\Delta \bar{q}_{3} \Delta \bar{q}_{4}}\right)\left(\bar{f}_{q_{1} q_{2}}+\bar{f}_{\Delta q_{1} \Delta q_{2}}\right)
\end{array}\right]
$$

with

$$
f_{q q}=f_{q^{+} q^{+}}+f_{q^{-} q^{-}}+f_{q^{+} q^{-}}+f_{q^{-} q^{+}}
$$

and

$$
f_{\Delta q \Delta q}=f_{q^{+} q^{+}}+f_{q^{-} q^{-}}-\left(f_{q^{+} q^{-}}+f_{q^{-} q^{+}}\right)
$$

where $q^{+}$is a quark with spin up, and $q^{-}$a quark with spin down.

Combined with the evolution of the parton density function as in [24] and the positivity bounds in [25] we can make a rough estimate of the relation between $f_{\Delta q \Delta q}$ and $f_{q q}$ for a given mass scale. At the $\mathrm{W}$ boson mass scale with x-values according to $\sqrt{s}=7 \mathrm{TeV}$ we find in the maximally polarized scenario:

$$
f_{\Delta q \Delta q} \approx \pm 0.2 f_{q q}
$$

In our treatment we investigate the positive sign relation in 1.17 and in that case we see that $F_{2}$ and $F_{3}$ are suppressed compared to $F_{1}$ and $F_{4}$, by a magnitude

$$
F_{(q q, \Delta q \Delta q)}=\left[\begin{array}{l}
1.44 \\
0.64 \\
0.64 \\
1.44
\end{array}\right] F_{q q}
$$

Taking a closer look at $A_{1}, A_{4}$ and $A_{2}, A_{3}$, we see that the former results in both muons having a preference for going to the same hemisphere in the detector, while the latter has a higher probability of the muons traveling through opposite hemispheres of the detector. This effect is shown in figure $1.4 \mathrm{using}$ as input parameter the rapidities of a $\mathrm{W}$ pair in the $\mathrm{W}$ boson rest frame, $\left(\eta_{W_{1}}=0\right.$, $\eta_{W_{2}}=0$ ). The histogram shows the probability for the rapidities of the outgoing muons in the lab frame. In the left plot $F_{2}$ and $F_{3}$ are set to zero, as are $F_{1}$ and $F_{4}$ on the right. Both histograms are centered around $(0,0)$, which stems from the input rapidity of the $\mathrm{W}$ pair. These input rapidities directly determine the center of the graph. In the left histogram where only $F_{1}$ and $F_{4}$ contribute, the outgoing muon rapidities have large probability of having equal sign, meaning the particles traverse the same hemisphere of the detector. The inverse is true in the right plot, resulting in two muons in opposite hemispheres.

A simulated event sample is generated using the Pythia [53] event generator. Pythia neglects spin correlations, and thus uses a factor $F$ as $F_{q q}$. A second set 

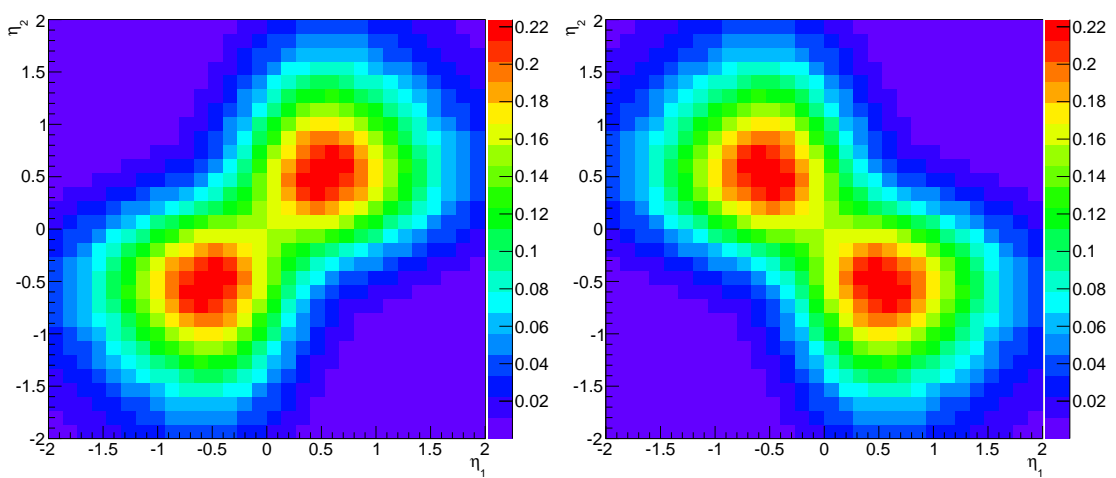

Figure 1.4: The rapidity distributions of the outgoing muons from a $\mathrm{W}$ pair created in a DPI process. The effect the weight imbalance introduced by equation 1.18 is emphasized: In the left plot the variables $F_{2}$ and $F_{3}$ are set to zero, as are $F_{1}$ and $F_{4}$ on the right. In the left figure the muons have a large probability of having an equal sign rapidity, indicated in red. Those muons will traverse the same hemisphere of the detector. The opposite is true for the muons in the right plot.

is derived from this which artificially adds spin correlations at their maximally polarized values $f_{\Delta q \Delta q}=0.2 f_{q q}$. This is achieved by adding a weight to the events in the initial set where both quarks originated from one proton and both antiquarks from the other with $w_{q q}=1.44$, and the events where both protons provide a quark and an antiquark with $w_{q \bar{q}}=0.64$. Cuts on lepton $p_{T}$, lepton rapidity, jet energy and multiplicity, and missing transverse momentum are used to obtain this sample, which are given in table 1.1

\begin{tabular}{|l|l|l|l|l|}
\hline muon $p_{T}$ & muon $\eta$ & $E_{\text {jet }}$ & nJet & $E_{T}^{\text {miss }}$ \\
\hline$>20 \mathrm{GeV}$ & $<5$ & $>20 \mathrm{GeV}$ & 0 & $>10 \mathrm{GeV}$ \\
\hline
\end{tabular}

Table 1.1: Cuts on the Pythia sample.

In table 1.2, the vanilla Pythia data set (the unpolarized hypothesis) has significantly less events where both final state muons travel through the same hemisphere of the detector, where the ratio of events in the same and opposite hemispheres in the maximally polarized hypothesis is almost unity. 

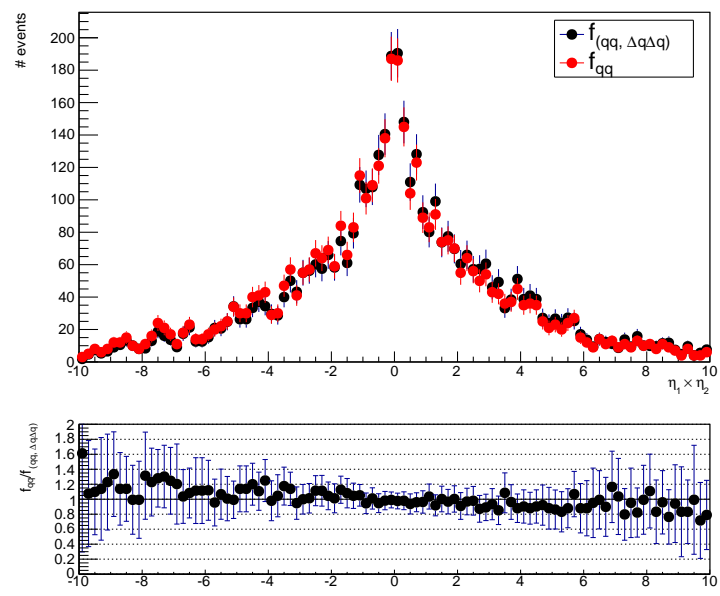

Figure 1.5: Effect of adding the maximally polarization hypothesis to the Pythia sample, compared to the vanilla Pythia sample. The graph shows the product of both muon rapidities. A positive value means both muons traversed the same detector hemisphere, a negative value is obtained when the muons travel through opposite hemispheres. On the bottom panel the ratio between the two sets is given. The negative slope in the ratio indicates that the maximally polarized hypothesis data set has a larger tendency to produce muons traversing the same hemisphere.

\begin{tabular}{|l|l|l|l|}
\hline hypothesis & same hemisphere & opposite hem. & same/opp \\
\hline unpolarized & $1991 \pm 45$ & $2183 \pm 48$ & $0.91 \pm 0.02$ \\
max polarized & $2175 \pm 47$ & $2133 \pm 46$ & $1.02 \pm 0.02$ \\
\hline
\end{tabular}

Table 1.2: Ratio of events with muons in same hemisphere or opposite hemisphere of the detector. The sample size is arbitrarily chosen based on available computing resources. Both data sets are generated using the Pythia event generator. The unpolarized set used the default parameters, where for the maximally polarized data set the weights from equation 1.18 are added. Note that when both interactions would be completely independent a same/opposite ratio of unity would be obtained. Only statistical errors are given in this table. 


\section{2 \\ THE LHC ACCELERATOR AND ATLAS DETECTOR}

The Large Hadron Collider (LHC) is the most powerful particle accelerating machine at the time of this writing. The data used in this study is recorded during most of 2012, when the machine was configured to accelerate two proton beams to $4 \mathrm{TeV}$. The two beams are stored in two rings, going in opposite directions. At certain points in the ring the two beams are intersecting, creating proton collisions at a center of mass energy of $8 \mathrm{TeV}$. This procedure is further described in section 2.1 .

To study the processes that result from a proton-proton collision huge detectors are built around the interaction points. The ATLAS detector [26] is one of two general purpose detectors in the LHC ring, the other being CMS [27]. ATLAS is a 10 storey high experiment aiming to record each particle that comes from a proton-proton collision, reconstructing the entire event. It also makes a decision on-line whether or not to store the event for later analysis. The detector is explained in more detail in section 2.2.

\subsection{Colliding protons with the LHC}

The LHC ring containing the experiments is the last step of an accelerator chain. At the beginning of this chain, a small bottle of hydrogen gas is connected to a linear accelerator called Linac2 [28]. Before the hydrogen enters Linac2 it is passed through an electric field to strip off its electrons. At the end of Linac2 the protons have reached an energy of $50 \mathrm{MeV}$, and are injected into a series of 


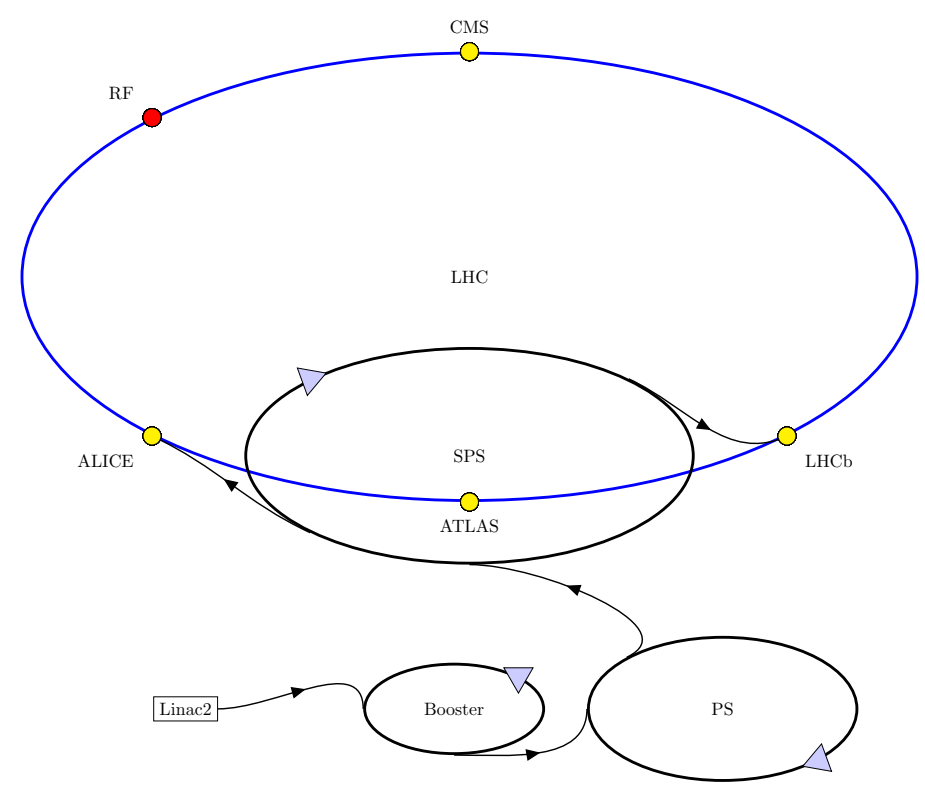

Figure 2.1: The accelerator chain of the LHC. In the linear accelerator Linac2 protons are extracted from hydrogen gas and accelerated to $50 \mathrm{MeV}$. The Booster, PS and SPS complex boost the energy of the protons to $450 \mathrm{GeV}$, after which they are injected in the LHC ring.

circular accelerators consisting of the Booster [29], Proton Synchrotron (PS) [30] and the Super Proton Synchrotron (SPS) [31]. After acceleration by the SPS, the protons have an energy of $450 \mathrm{GeV}$, high enough to enter the LHC. The SPS also divides the beam into a so called bunch train, small bunches of protons separated 50ns apart. Exact timing of the bunches is crucial to have the bunches collide at the centers of the experiments. This timing information is also provided to the experiments, so they can coordinate their subsystems to start recording at the correct moment.

The bunch trains are further accelerated in the LHC ring using a series of $16 \mathrm{RF}$ cavities located at Point 4 [32], until the maximum energy is reached. The rest of the 27 kilometer ring consists of 1232 dipole magnets [33] to direct the beam into orbit, and quadrupole and sextupole magnets to focus the beam. During 2012, when the data for this study was taken, beam energy was $4 \mathrm{TeV}$. When this energy level is achieved the LHC switches to storage ring mode.

Two key parameters of an accelerator are the luminosity $\mathcal{L}$ and the center of mass energy $\sqrt{s}$. The luminosity is simply the ratio of the number of events $N$ 
detected per unit time $t$ to the event interaction cross section $\sigma$.

$$
\mathcal{L}=\frac{1}{\sigma} \frac{d N}{d t}
$$

It only depends on the beam characteristics, like the collimation and the number of particles per bunch. It is therefore more useful from a physics point of view to refer to the integrated luminosity, which directly translates to the number of events in the data for a given production cross section. The integrated luminosity delivered by the LHC to the experiments in 2012 was $20.3 \mathrm{fb}^{-1}$. This means a process with a cross section of 5 femtobarn has occurred on average 100 times.

The center of mass energy $\sqrt{s}$ gives the theoretical upper limit for mass of the possible resonances that can be produced. The LHC beam energy in 2012 was $4 \mathrm{TeV}$, giving the aforementioned $\sqrt{s}=8 \mathrm{TeV}$. Since the proton is a composite particle, and the constituents have to share the full momentum of the proton, the mass upper limit of new particles that can be produced with the accelerator is of the order of $1 \mathrm{TeV}$.

There are four interaction points in the LHC ring. At these points the multipole magnets steer the two anti-parallel beams through each other, causing the protons to collide. The timing is crucial here since the beams are divided in bunches. One bunch from one beam has to pass the interaction point at exactly the same time as a bunch from the other proton beam. During such a so called bunch crossing not only one proton collision takes place, but on average $<\mu>=20.7$ for the 2012 data taking period. Figure 2.2 shows the distribution of interactions per bunch crossing in the 2012 proton-proton data set.

\subsection{Studying collisions with the ATLAS detector}

As stated before, the ATLAS detector is a giant, cylindrical machine measuring over 45 meters in length and 25 meters in diameter. It is designed to fully enclose the interaction point in order to calculate the full energy transfer in the collision. This is particularly useful to detect particles that do not leave any trace in the detector. Using conservation of momentum an imbalance in the transverse momentum sum of all detected particles means that one or more unseen particles were produced as well. This is only possible in the transverse direction because the longitudinal momentum components of the colliding partons is unknown. The longitudinal momentum $p_{z}$ of the protons is known to great precision, but the fraction carried by their partons is determined by the probability distributions discussed in the previous sections.

The following description uses the trigger as a guide as it makes its decision whether or not to record the current event. Every subsystem is described when the trigger system requests data from it. 


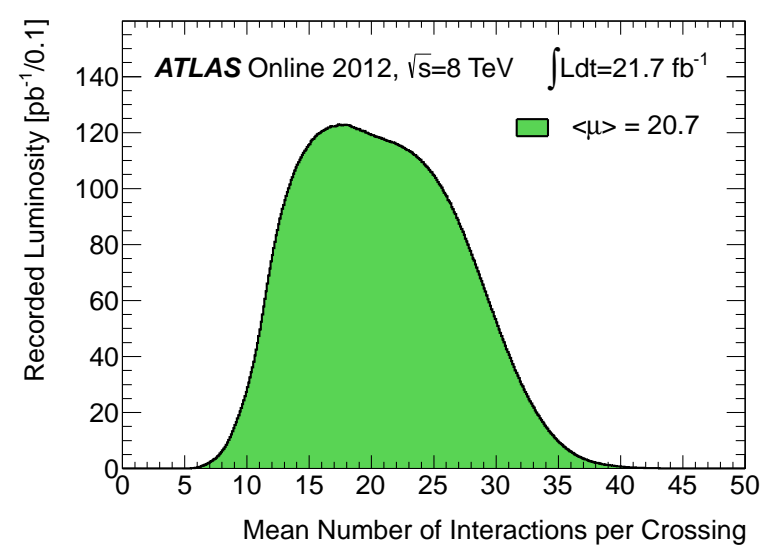

Figure 2.2: The luminosity-weighted distribution of the mean number of interactions per bunch crossing in 2012 (full pp collisions data set). The average number of interactions over all physics grade collisions in 2012 was $\langle\mu\rangle=20.7$.

\subsubsection{The ATLAS coordinate system}

The origin of the detector coordinate system is the nominal interaction point; the central point in the detector where the collisions take place. The positive $\mathrm{x}$-axis is pointing from the interaction point to the center of the LHC ring. The positive $\mathrm{y}$-axis is pointing upward. The z-axis is aligned with the beam pipe, with the positive direction counterclockwise as seen from the center of the LHC.

Due to the shape of ATLAS a cylindrical coordinate system is used with $\phi$ the azimuthal angle in the transverse plane and $\theta$ the polar angle. This polar angle is used in the definition of a important variable: the pseudorapidity $\eta$.

$$
\eta=-\ln [\tan (\theta / 2)]
$$

The pseudorapidity is preferred over the polar angle in collision studies as the particle multiplicity is roughly constant as a function of pseudorapidity. The angle corresponding to $\eta=0$ is perpendicular to the beam pipe and approaches infinity as the angle tends towards $90^{\circ}$.

\subsubsection{Event selection: The ATLAS trigger system}

The trigger system in ATLAS is a three-stage decision mechanism, designed to capture the physics of interest from a $40 \mathrm{MHz}$ bunch crossing rate. Each level improves the decision made at the previous stage, and if applicable adds additional 


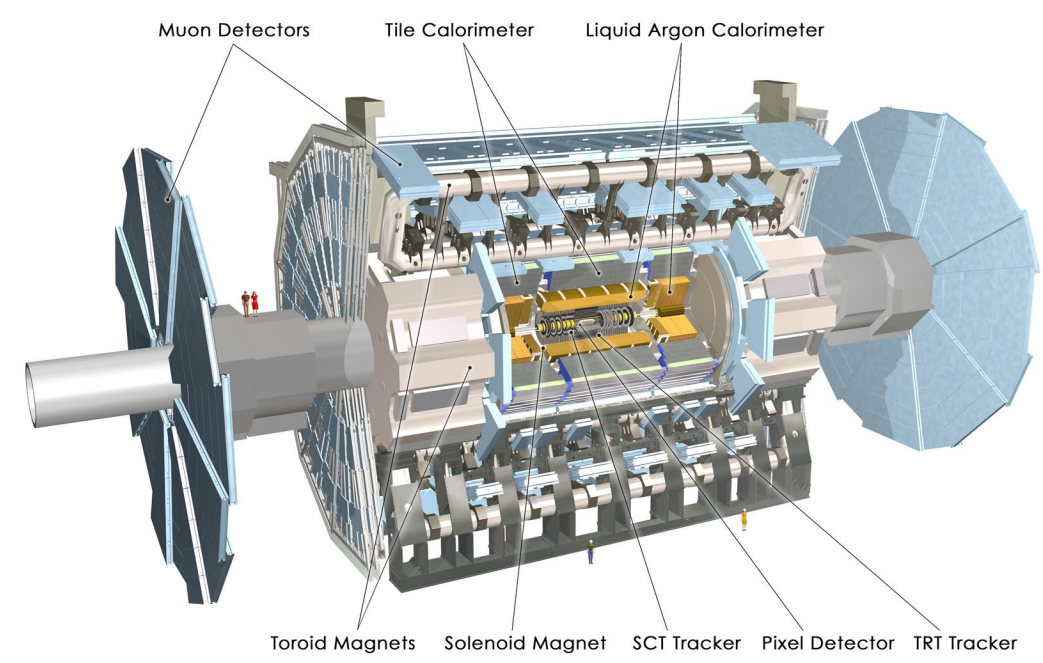

Figure 2.3: Computer generated model of the ATLAS detector. The full size of the machine is 45 meters in length and 25 meters high. The beam pipe enters from the left and right sides. The muon system and the calorimeter locations are indicated at the top, the magnet system and inner tracker subsystems are indicated at the bottom of the figure.

selection criteria. After the third selection level the output frequency should be in line with the storage rate capacity of the back-end database, which is designed to be $200 \mathrm{~Hz}$.

The three decision levels are called Level 1 (L1) [34], Level 2 (L2) and Event Filter (EF). L1 is fully hardware based using ASICs and FPGAs, L2 and EF are jointly called the High Level Trigger (HLT) [35] and is run in software on a large computing farm.

In between the data taking periods in 2012 an intermediate level was added to the trigger, The Level 1.5 jet trigger (L1.5) [36]. In contrast to the normal L2 trigger the L1.5 trigger uses data from the entire calorimeter of the detector, instead of looking only in a small area in $(\eta, \phi)$ space called a Region of Interest (RoI) provided by L1.

\section{First decision: Level 1}

The Level 1 trigger makes the initial selection based on information from a subset of detectors. High $p_{T}$ muons are identified in the Resistive Plate Chambers (RPCs) in the barrel and Thin Gap Chambers (TGCs) in the endcap regions 
[37]. The L1 hardware also has access to reduced granularity info from the calorimeter, both electronic (EM) and hadronic, in the barrel, endcap and forward regions $[38,39]$. The $\mathrm{L} 1$ calorimeter algorithms are searching for high $p_{T}$ electrons and photons, jets, $\tau$ particles decaying into hadrons, and large $E_{T}^{m i s s}$ and large transverse momentum.

\section{Muon system}

Muons have the ability to traverse large lengths of material, so the muon system is the outermost subsystem in the detector. All the muon subsystems are collectively called the Muon Spectrometer (MS), and two subsystems of the MS are connected to the L1 trigger due to their fast response time. In the barrel region three layers of Resistive Plate Chambers are measuring the track of passing muons. A powerful magnet bends the shape of the muon path, thereby providing an energy measurement if all three layers detected the particle. The RPCs are gaseous detectors with an high electric field through the active gas, which is provided by two resistive plates. These plates are covered with readout strips that detect ionization avalanches induced by the passing muons. The end-cap muon trigger is constructed using of Thin Gap Chambers. Like the RPCs the TGCs are gaseous detectors using high voltage and readout strips to measure ionization avalanches, but unlike the RPCs the high voltage is not on large resistive plates but on thin wires running through the active medium.

\section{Calorimeters}

The full calorimeter system forms a closed structure around the interaction point so it is able to make a complete measurement of the interaction energy. This enables us to measure the full sum of particle momenta produced by the collision, but more importantly also the transverse energy imbalance, or missing transverse energy $E_{T}^{\text {miss }}$, caused by particles that traverse the detector unseen. These particles are in most cases neutrino's for which this is the only way to measure them. In our study there are two neutrino's in the final state, making $E_{T}^{\text {miss }}$ a very important variable.

The data sent from the calorimeter systems to the trigger has a typical granularity of $\Delta \phi \times \Delta \eta=0.1 \times 0.1$, which becomes larger beyond $|\eta|=2.5$. Trigger towers are constructed in the front-end calorimeter electronics by summation over the corresponding EM and hadronic cells. Figure 2.4 shows the location in pseudorapidity of the Liquid Argon and Tile calorimeters. The hadronic calorimeters are everywhere outside the EM calorimeters because of the higher stopping power of the latter.

The thickness of a calorimeter is determined by the nuclear interaction length $\lambda$ and the radiation length $X_{0}$ of the material used. The nuclear interaction 


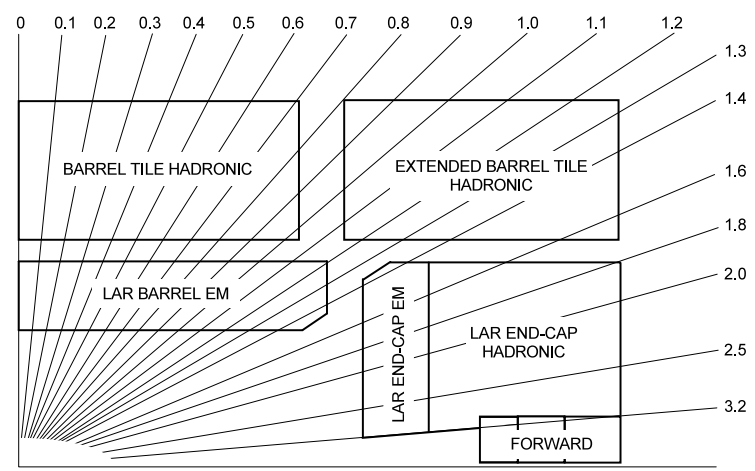

Figure 2.4: The pseudorapidity positions of the Liquid Argon calorimeters and the hadronic Tile calorimeters. The values on the side of the diagram are given in units of rapidity $\eta=-\ln [\tan (\theta / 2)]$. Notice that the two EM calorimeters are positioned in front of the three hadronic calorimeters. The forward calorimeter is the subsystem closest to the beam pipe, and is therefore designed to operate in the highest rate environment.

length is defined as the mean distance traveled by an hadron before in interacts inelastically with a nucleus, and is thus largely dependent on the atomic number $Z$ of the traversed material. The radiation length is the mean distance over which an high energy electron loses all but $1 / e$ of its initial energy. This is also dependent on the atomic number of the medium, but equally on the mass number $A$ because that is related to the number of electrons carried by the traversed material.

The EM calorimeter uses liquid Argon as active medium, and lead plates as absorbers. The absorbers are accordion shaped to have a $\phi$ coverage without gaps. A passing particle creates free charges in the liquid argon, and the current induced by this process is read out by electrodes between the lead absorbers. The thickness of the EM calorimeter is designed for $\lambda_{E M c a l} \approx 1.5$ and $X_{0, E M c a l} \in$ $(22,38)$ to stop all EM showers while letting the hadronic activity pass through to the hadronic calorimeter.

The Tile calorimeter is positioned outside the EM calorimeter and is designed to measure the hadronic energy in the events. It derives its name from the plastic scintillator tiles used as active material. When a particle traverses one of these tiles, scintillation photons are produced in the material which are picked up by photomultiplier tubes (PMTs). The hadronic calorimeter in the end-caps are Liquid Argon detectors, similar to the EM calorimeter. The depth of the hadronic calorimeters is about $\lambda_{\text {Hcal }} \in(7.5,10)$ to ensure that most hadronic activity is 
contained. This is important to minimize punch through to the subsystem outside the hadronic calorimeter, the muon spectrometer.

The forward calorimeter is closest to the beam pipe and receives the highest amount of radiation. It covers the $3.1<|\eta|<4.9$ region and is important in the reconstruction of missing transverse energy $E_{T}^{\text {miss }}$. The calorimeter is divided into three subsystems, of which the first already stops all EM showers with a $X_{0}=28$ and all three modules combined have a $\lambda=10$.

\section{Level 1 output}

Before the signals from the muon trigger chambers and the calorimeters can enter the Level 1 Central Trigger Processor (CTP), it goes through a preprocessing stage. The signals from the muon chambers readout strips is converted into a Region of Interest (RoI) and a multiplicity of muons per $p_{T}$ threshold. The calorimeter towers are first converted into RoIs by two trigger processors. The Cluster Processor finds and counts energy cluster corresponding to pass-through of electrons, photons and tau particles. The Jet/Energy-sum Processor measures the total $E_{T}$ and $E_{T}^{m i s s}$ in the full calorimeter.

Based on the various particle multiplicities with $p_{T}$ thresholds and energy variables from the calorimeter, a final Level 1 Accept decision is made. When the event is accepted, all RoIs and particle information is send to readout buffers including a detailed description why the event is accepted. Level 2 is now signaled to start processing this data further and more importantly continue to read out other detector subsystems.

\section{Second decision: Level 1.5 and Level 2}

The Level 2 trigger stage is built in software running on a farm of PCs. The main purpose of this level is to perform a local analysis of the L1 candidate, using extra data from the detector. This additional information makes a more fine-grained energy profile from the calorimeter possible, but the most important inclusion are the reconstructed tracks from the Inner Detector (ID) [40]. The Level 1.5 jet trigger searches for jet signatures in the whole $\eta, \phi$ space, in contrast to the common L2 trigger which works in seeded mode, only using data inside the RoIs provided by L1.

\section{Inner detector}

The detector system closest to the interaction point is aptly called the Inner Detector. It measures the exact location of and tracks emanating from the points where two partons interact. This part of the detector is subject to the highest particle flux, and it's target on momentum and vertex resolution require a fine 
granularity. Semiconductor strip and pixel detectors [41,42] provide these features and are used closest to the beam pipe. Around these two subsystems a Transition Radiation Tracker (TRT) [43] is installed to add additional data points to the tracking algorithm. The TRT consists of straw detectors, a thin tube filled with Xenon gas and a anode wire in the middle. The straw itself and the anode wire are on a potential difference, and when high energy charged particles ionize the gas a signal is induced in the anode wire.

When the ID fails to separate two vertices due to accuracy constraints, shadowing occurs. Shadowing means that a pile-up interaction took place very close to the hard-scatter signal event, where the ID fails to separate the two. This is therefore determined by the $z$ accuracy of the ID, the number of pile-up events per bunch crossing and the efficiency of the vertex finding algorithm. The mean number of events per bunch crossing in 2012 was $\langle\mu\rangle=20.7$ as can be seen in figure 2.2 in the previous section. Using the $z$ resolution of 115 (580) $\mu \mathrm{m}$ of the pixel (strip) semiconductor detectors [26] and an average bunch length of $75 \mathrm{~mm}$ the probability of the primary vertex being shadowed by a pile-up vertex is:

$$
P_{\text {overlap }}=<\mu>\frac{2 \times 0.58}{75}=0.32
$$

Previous measurements [44] show that the ID has difficulty distinguishing vertices that are less than $2 \mathrm{~mm}$ apart, giving an $P_{\text {overlap }} \approx 0.5$.

\section{Final decision: Event Filter}

The Event Filter (EF) makes the final decision to write the event out to the event storage facility. The output rate is limited by the speed of the permanent storage system to about $200 \mathrm{~Hz}$. The processing farm where the Event Filter is running is designed to provide a time available to reach a decision of about four seconds per event. The EF has full access to all the detector subsystems, and is designed to run the same event building algorithms as the offline reconstruction.

\section{Trigger chains and streams}

The three trigger levels are chained together in so called trigger chains. The trigger chains are sequences of 2 to 10 algorithms reconstructing mostly objects in the RoI selected by L1. An example of a trigger chain used in this analysis is the EF_mu24i_tight chain. The name refers to the algorithm run at Event Filter, and is seeded by the L2_mu24_tight algorithm at Level 2, which is in turn seeded by L1_MU15 at Level 1. Multiple HLT algorithms can be seeded by the same Level 1 rules, for example the other muon chain used in this analysis, EF_mu36_tight, is also initially seeded by L1_MU15. Similar trigger chains are recorded in the same data set using trigger streams. ATLAS has four such streams: E $\gamma$, Muon, 
Jet/Tau $/ E_{T}^{\text {miss }}$ and Minimum Bias. Overlap between streams is minimized. All chains in the streams are combined in the trigger menu, which contained over 700 entries in 2012.

\subsubsection{Event reconstruction: ATLAS performance}

With ATLAS being a multi purpose detector, there is no unanimous performance figure for the machine. The performance and precision is highly dependent on the process under study in the analysis. Since the study described in this thesis has a focus on $\mathrm{W}$ bosons with muons and missing energy in the final state, the detector performance will rely on the single muon and calorimeter efficiencies. These quantities are studied in the following sections, starting with the muon performance and the calorimeter performance, concluding with a $\mathrm{W}$ reconstruction performance using Monte Carlo samples generated with Alpgen [45].

\section{Muon resolution}

The muon detection resolution of ATLAS can be divided into two components: the resolution on the direction of traversal and a resolution on the muon kinetic energy. The resolution on muon direction is determined by the resolution of the Inner Detector, plus the track reconstruction resolution of the Inner Detector and the Muon Spectrometer combined. Given the sub-millimeter resolution of the ID and the combined track reconstruction with the MS only tightening this number the muon direction resolution of ATLAS is of micrometer scale. The energy reconstruction resolution is usually given as the uncertainty of the so called muon energy scale. This scale is a multiplication factor on the measured momentum to obtain the true momentum of the muon, and should ideally be close to unity. A momentum scale lower than 1 indicates the detector is overestimating the muon energy, a scale above 1 means an underestimated momentum. Figure 2.5 shows the uncertainty on the momentum scale correction in both the Inner Detector and the Muon Spectrometer to be less than $0.4 \%$ in the entire rapidity region.

\section{Muon efficiency}

The ATLAS collaboration reports [46] a muon reconstruction efficiency above $99 \%$, measured in large reference samples of $J / \Psi \rightarrow \mu \mu, Z \rightarrow \mu \mu$ and $\Upsilon \rightarrow \mu \mu$ decays. Muons are reconstructed in ATLAS using data from the Muon Spectrometer, the Inner Detector and to a lesser extent the calorimeters. The efficiency of muon reconstruction highly depends on which subsystems the particle traversed. If the muon is reconstructed in both the ID and MS independently it has the 

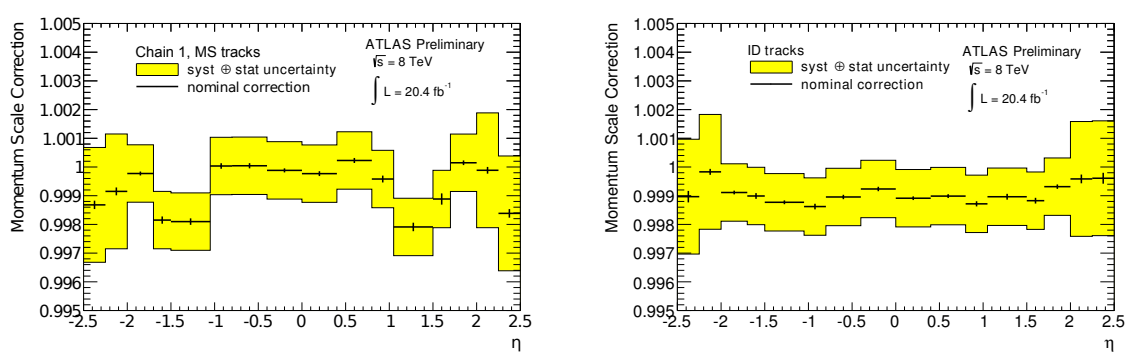

Figure 2.5: Momentum scale derived from $Z \rightarrow \mu \mu$ data as a function of rapidity. The systematic uncertainty on the correction is shown in yellow, which is treated as the energy reconstruction resolution. The figure on the left is the momentum scale for MS tracks, which shows the spectrometer to have an uncertainty on the momentum resolution within $0.4 \%$. The right plot is for the Inner Detector tracks which have similar resolution. Figures taken from [47].

highest efficiency and is called a Combined (CB) muon. However, the MS has regions of reduced acceptance due to the support structure of the detector. Adding Segment-Tagged (ST) muon reconstruction, muons that are reconstructed by the ID and can be associated with local track segments in the MS, will increase the muon efficiency further.

The muon reconstruction efficiency is measured in the reference samples using the "tag-and-probe" method. Basically the ID efficiency is measured using muons reconstructed in the MS and vice versa, giving

$$
\epsilon_{\mu}=P(M S \mid I D) \cdot P(I D \mid M S)
$$

Events are selected by requiring two oppositely charged isolated muons. One muon, the tag, is required to be a CB muon and matched to a muon reconstructed in the trigger. The other muon, the probe, has to be anti-parallel to the tag $(\Delta \phi>2)$ and have an MS track to measure $P(I D \mid M S)$, or have an ID track and a minimum ionizing signature in the calorimeter to measure $P(M S \mid I D)$. The result of the tag-and-probe analysis is shown in figure 2.6 and 2.7. Figure 2.6 shows a reconstruction efficiency around $99 \%$ for the whole transverse momentum range. Most relevant is the $\mathrm{Z}$ boson data, since our study is done with muons having a $p_{T}>10 \mathrm{GeV}$. The muons with very high $p_{T}$ have a growing uncertainty on efficiency due to the lack of statistics in the high end of the spectrum. Figure 2.7 shows a reconstruction efficiency around $99 \%$ for pile-up conditions lower than 30 . Since the average number of pile-up vertices in the data used in our analysis is $\langle\mu\rangle=20.7$, this decay of efficiency due to high pile-up is of minor importance in this context. Both figures indicate a muon efficiency around $99 \%$ for the muons 


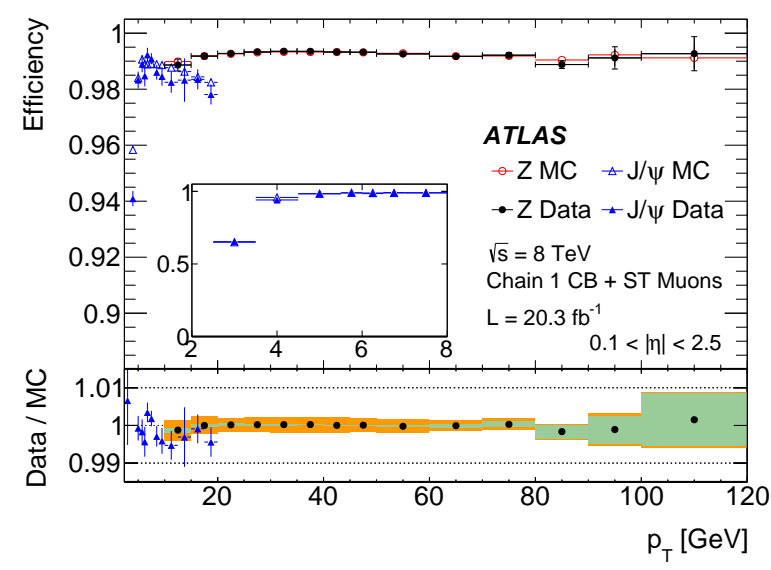

Figure 2.6: Reconstruction efficiency for muons as a function of transverse momentum. The blue data points are from the $\mathrm{J} / \Psi$ data (low energy muons), and the black points from the $\mathrm{Z}$ boson data. The ratio between the measured and predicted efficiencies is shown in the bottom panels, with the green area indicating only statistical error, and yellow area the systematic error. Figure taken from $[46]$.

used in this analysis, with slight degradation in higher pile-up conditions. The next section explores the efficiency degradation over time during the 2012 data taking period.

\section{Muon reconstruction: performance degradation over time}

The previous section showed a slight decay in efficiency for higher pile-up conditions. Reduced performance is also expected after long periods of data taking due to wear and tear of the detector. The following study is an attempt to identify muon reconstruction efficiency degradation over time.

The efficiencies obtained in the study described in the previous section are measured per Data Period. The ATLAS data taking in 2012 is divided into 10 data taking periods. A data set is constructed with all the muons found in these Data Periods, using the same baseline cuts on reconstruction as in the main analysis of $\mathrm{W}$ pair production in DPI events. For every muon found the reconstruction efficiency is determined, and these efficiencies are averaged over the whole period, giving an average muon reconstruction per Data Period. The results are shown in table 2.1. The figures in the table show no degradation in efficiency, indicating a close to $99 \%$ mark on average stable over all ten periods. 


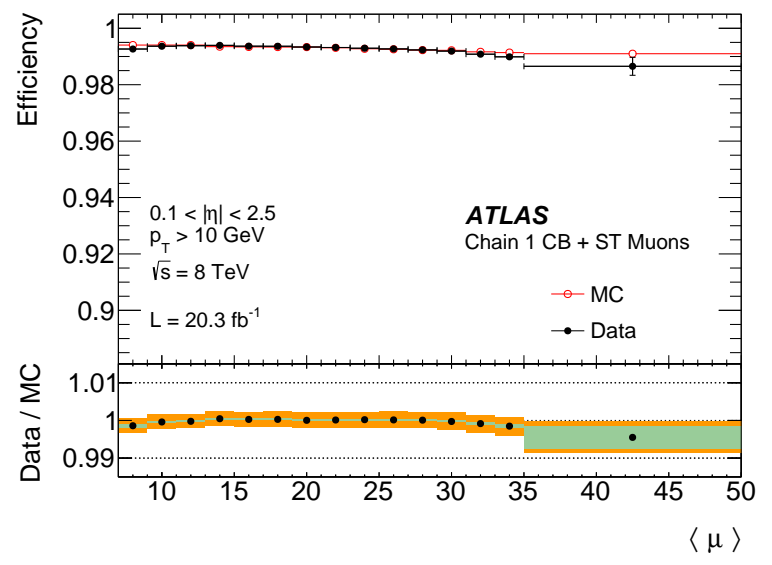

Figure 2.7: Reconstruction efficiency for muons as a function of pile-up. Efficiency seems to drop for high pile-up conditions. This drop sets in after $\langle\mu\rangle=30$, where the average number of pile-up vertices in the data used in this analysis is $\langle\mu\rangle=20.7$. The ratio between the measured and predicted efficiencies is shown in the bottom panels, with the green area indicating only statistical error, and yellow area the systematic error. Figure taken from [46].
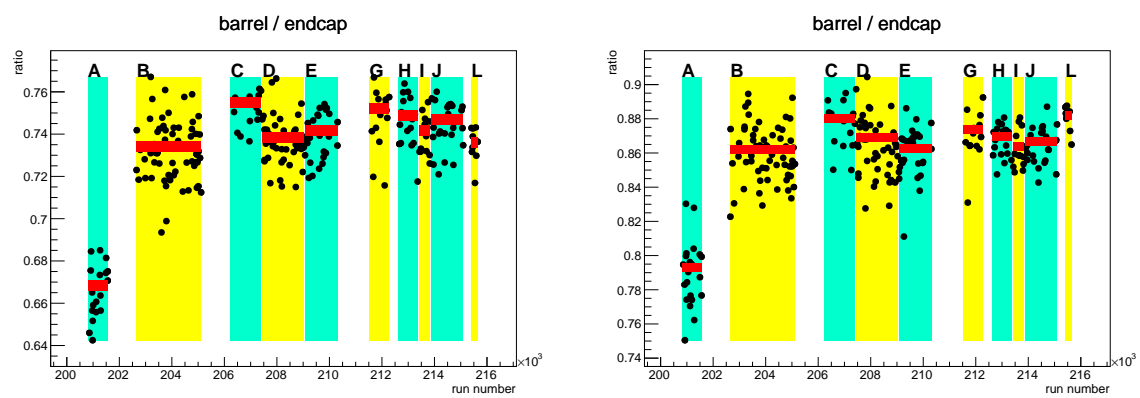

Figure 2.8: Per run ratio of reconstructed muons in the barrel region $(|\eta|<1)$ and the end-cap region $(1.4<|\eta|<2.5)$. The left figure shows the ratio for $\mu^{+}, \mu^{-}$is shown on the right. The lower ratio found in period $\mathrm{A}$ is due to a change in trigger menu. The lower ratio for positive muons is because a larger fraction is produced by valence quarks which carry a higher momentum fraction of the proton. The ratios in periods $\mathrm{B}$ to $\mathrm{L}$ show no trending increase or decline of the barrel/end-cap ratio, indicating the absence of a significant efficiency deterioration. 


\begin{tabular}{|r|l|l|l|l|l|}
\hline Period: & $\mathrm{A}$ & $\mathrm{B}$ & $\mathrm{C}$ & $\mathrm{D}$ & $\mathrm{E}$ \\
\hline$\left\langle\epsilon_{\mu^{+}}\right\rangle$ & 0.990 & 0.989 & 0.988 & 0.989 & 0.988 \\
$\left\langle\epsilon_{\mu^{-}}\right\rangle$ & 0.989 & 0.987 & 0.987 & 0.987 & 0.988 \\
\hline \hline Period: & $\mathrm{G}$ & $\mathrm{H}$ & $\mathrm{I}$ & $\mathrm{J}$ & $\mathrm{L}$ \\
\hline$\left\langle\epsilon_{\mu^{+}}\right\rangle$ & 0.988 & 0.988 & 0.989 & 0.989 & 0.989 \\
$\left\langle\epsilon_{\mu^{-}}\right\rangle$ & 0.988 & 0.987 & 0.987 & 0.987 & 0.988 \\
\hline
\end{tabular}

Table 2.1: Average muon reconstruction efficiencies per Data Period. Efficiencies are slightly different for positively and negatively charged muons.

The ratio of number of muons recorded in the barrel region and the end-cap regions can also be an indication that in either two regions an efficiency loss occurred due to component failure. Figure 2.8 shows the ratios per run for the positively charged muons and the negatively charged muons.

Two characteristics of this figure need further explaining: the absolute magnitude difference between $\mu^{+}$and $\mu^{-}$, and the lower ratio for period A. Starting from period $\mathrm{B}$ the trigger menu switched to an isolated muon trigger with a different efficiency as a function of rapidity than the muon trigger used in period A. This accounts for the outlier position of period A. The lower barrel/end-cap ratio for positive muons compared to negative muons is because the positive charge of the protons. Due to this there are twice as many positively charged valence quarks then there are negative in the proton. Positive muons are more often produced by valence quarks due to this, and since valence quarks carry a larger momentum fraction of the proton than sea quarks at the momentum transfer regime in this study, the positively charged muons have on average a higher $p_{z}$ compared to negatively charged muons. Comparing the ratios of reconstructed muons in the barrel and end-cap regions in periods B to L, there is no sign of a trending increase or decline of the ratio. Hence this study does not indicate an efficiency deterioration located in either only the barrel or only the end-cap region.

The lower ratio found in period $\mathrm{A}$ is due to the fact that starting from period $\mathrm{B}$ the trigger menu switched to an isolated lepton trigger, the difference between negative and positive muons is because positive muons are more often created using a valence quark, resulting in higher $p_{z}$ on average. The data in figure 2.8 does not show any sign of deterioration, which would manifest itself in a sloped average.

\section{Calorimeter efficiency}

The full calorimeter system forms a closed structure around the interaction point so it is able to make a complete measurement of the interaction energy. The 
input momentum from the protons is entirely longitudinal, so if particles escapes the detector unseen it will likely cause a transverse energy imbalance, or missing transverse energy $E_{T}^{\text {miss }}$. These particles are in most cases neutrino's for which this is the only way to measure them. The missing transverse momentum is an important variable in the event selection for this study. This treatment of calorimeter efficiency is focused on the ability to correctly measure this quantity.

The measurement of $E_{T}^{\text {miss }}$ is notably affected by pile-up due to the extra activity in the calorimeters induced by the additional processes. Several data samples are used to estimate the $E_{T}^{\text {miss }}$ performance [48], including leptonically decaying $\mathrm{W}$ bosons. The performance figures obtained with this data sample is the focus of the following discussion since our analysis uses the same final state.

The calculation of the missing transverse momentum uses calorimeter deposits that are associated with an identified high $p_{T}$ physics object as well as unassociated energy deposits from topological clusters and tracks. Energy not associated with high $p_{T}$ objects is called the soft term $E_{T}^{\text {miss,SoftTerm }}$. Energy deposits that end up in the soft term usually come from low energy particles from the underlying event. The full calculation of $E_{T}^{m i s s}$ is the Pythagorean combination of the following components:

$$
E_{x(y)}^{m i s s}=\sum E_{x(y)}^{m i s s, \text { obj }}, \quad \text { obj } \in(e, \gamma, \tau, \text { jets, } \mu, \text { SoftTerm })
$$

where each term is the negative of the reconstructed physical object, projected on the $x$ and $y$ directions. All terms are affected by pile-up, but the jets and soft term contributions are influenced most due to their dependence on hadronic energy.

The scalar sum of all the transverse energy in the calorimeters, $\sum E_{T}$, is a useful variable to evaluate the $E_{T}^{\text {miss }}$ performance:

$$
\sum E_{T}=\sum E_{T}^{m i s s, \text { obj }}, \quad \text { obj } \in(e, \gamma, \tau, \text { jets, SoftTerm })
$$

The total transverse energy in the event is the sum of the muon transverse momenta and $\sum E_{T}$ :

$$
\sum E_{T}(\text { event })=\sum E_{T}+\sum p_{T}^{\mu}
$$

The effect of pile-up is mitigated using two techniques. The first technique uses the track information from the Inner Detector to determine if the calorimeter activity is caused by the hard scatter or a pile-up collision. The second method is based on the energy distribution in the jet reconstruction area, and the final energy given to the reconstructed jet is corrected for additional activity in this area due to pile-up. In the left plot in figure 2.9 the $E_{x(y)}^{m i s s}$ resolution is given as a function of calorimeter total transverse energy. The pile-up reduction techniques (jet area and track STVF) performance is as shown. The range most relevant 

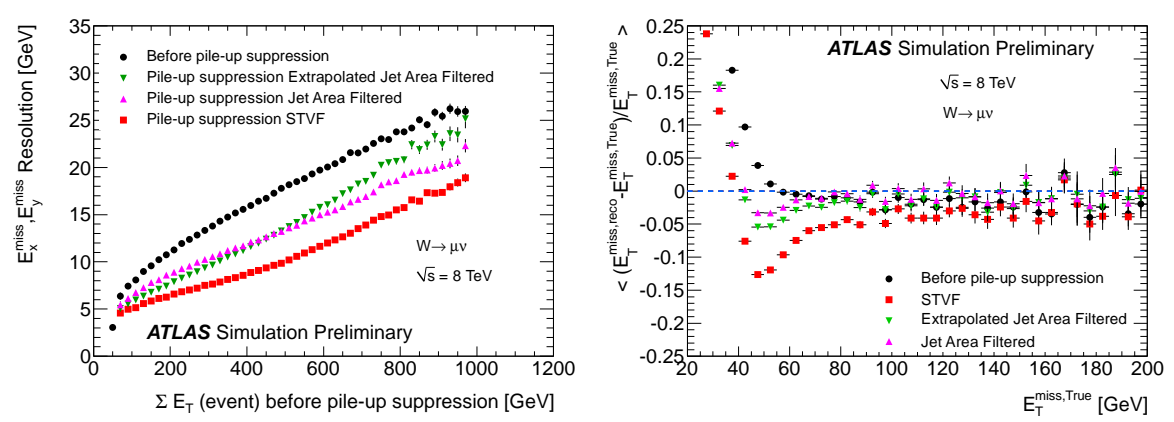

Figure 2.9: Left: $E_{T}^{\text {miss }}$ resolution as a function of total transverse energy. Right: $E_{T}^{m i s s}$ linearity as a function of actual $E_{T}^{\text {miss }}$ from all non-interacting particles. It gives the fraction of energy in $E_{T}^{\text {miss }}$ originating from particles that actually do leave a signal the calorimeter. Data in both figures is from in simulated $W \rightarrow \mu$ events. Taken from [48].

in the study described is $\sum E_{T}<200 \mathrm{GeV}$, where the data shows a resolution of $\pm 5 \mathrm{GeV}$ in this limit. The right plot in the figure is the $E_{T}^{m i s s}$ linearity, which is defined as the mean value of the ratio:

$$
\operatorname{linearity}\left(E_{T}^{\text {miss }}\right)=\left(E_{T}^{\text {miss }}-E_{T}^{\text {miss }, \text { True }}\right) / E_{T}^{\text {miss,True }}
$$

where $E_{T}^{m i s s, T r u e}$ is the actual $E_{T}^{m i s s}$ calculated from all non-interacting particles, most importantly neutrinos, in the event. This means that this variable can only be studied in simulated events. A large non-linearity is observed for track based pile-up mitigation (STVF), because STVF has the largest effect on pile-up in events without jets. Most events however have a missing transverse energy around $40 \mathrm{GeV}$, where linearity is between $7 \%$. The most important test of the $E_{T}^{\text {miss }}$ performance is the validation of the transverse mass $m_{T}$ calculation, because this is an important variable in the study described in this document. Figure 2.10 shows the transverse mass performance as a function of $m_{T}^{\text {True }}$ and $E_{T}^{\text {miss, True }}$. Later in this document it will be shown that the bulk of the signal events for this study has a missing transverse energy in the range of 30 to $70 \mathrm{GeV}$. The right figure shows a small bias of 0 to $5 \mathrm{GeV}$ on the calculation of $m_{T}$ in this interval.

\section{ATLAS performance conclusion}

In later chapters the muon requirements will be given to be in the transverse momentum range of $p_{T}<10 \mathrm{GeV}$ and rapidity $\eta<2.5$. The direction of these muons is not of great importance, since the rapidity cut is far from the beam 

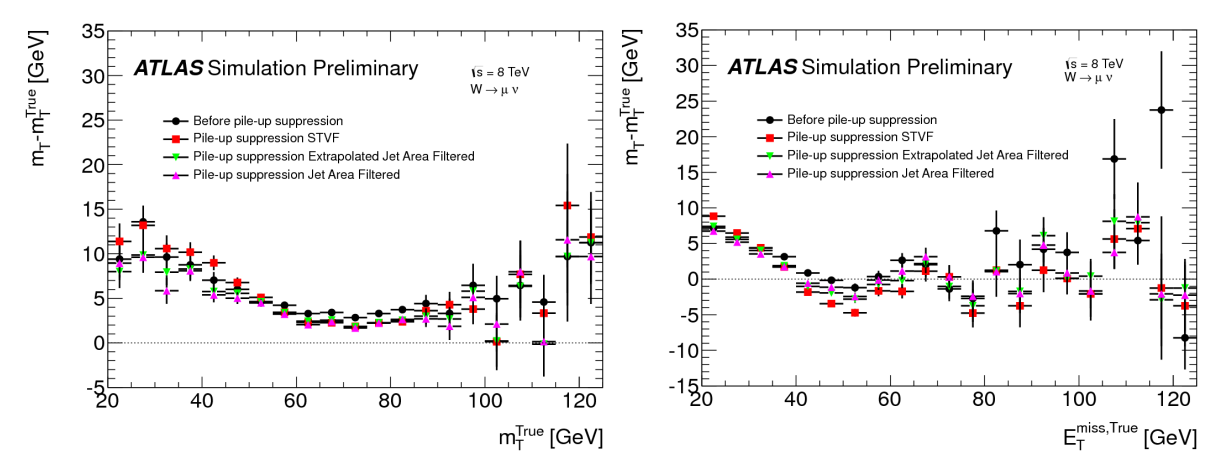

Figure 2.10: Transverse mass performance as a function of $m_{T}^{\text {True }}$ (left) and $E_{T}^{\text {miss,True }}$ (right). Data in both figures is from simulated $W \rightarrow \mu$ events. Performance is defined as the difference between true transverse mass as calculated from the output of the event generator, and the reconstructed transverse mass as calculated from the output of the detector simulation and event reconstruction algorithms. Taken from [48].

pipe. The direction resolution of ATLAS is in the micrometer range, orders of magnitude better than necessary for our study. The transverse momentum resolution for those muons is of the order of $0.4 \%$, and the detection efficiency is around $99 \%$.

The $E_{T}^{\text {miss }}$ requirement in our analysis is $E_{T}^{\text {miss }}>10 \mathrm{GeV}$, with the bulk of events having $30 \mathrm{GeV}<E_{T}^{\text {miss }}<70 \mathrm{GeV}$. The performance plots show a bias between 0 to $5 \mathrm{GeV}$ in this interval, corresponding to a linearity, or deviation of true value, of around $7 \%$.

\subsubsection{Noise reduction in the jet trigger}

Jets are the most numerous physics objects in the output data set of the detector. It is therefore important to have a good noise reduction in the jet trigger, to avoid recording useless data. The jets are mostly recorded in the calorimeter, and false readings in the calorimeter subsystems can trick the reconstruction algorithm into thinking a jet was created in the collision. False readings usually come from electronic noise in a particular subsystem, and they can be effectively filtered out by taking a closer look at the total jet reconstruction algorithm and the variables this procedure supplies.

Jet reconstruction in the ATLAS trigger starts at L1. The L1 trigger creates Jet Elements (JE) from reconstructed energy depositions in the calorimeter. These JEs have a basic granularity of 0.2 in $\Delta \eta$ and $\Delta \phi$ (some JEs in the outer 
regions of $\eta$ are $0.3 \times 0.2$ ), and give the energy sum of both the EM and the hadronic calorimeters in the region specified by the JE. These JE energies are summed in a $2 \times 2,3 \times 3$ or $4 \times 4$ cluster of JEs to identify L1 jet candidates.

When L1 finds a jet candidate, it provides the location of the JEs to the L2 stage, creating an RoI. The L2 trigger uses a simple cone algorithm to try to reconstruct jets from calorimeter cells within the RoI, or in case of L1.5 all the cells in the detector are used. From this cone algorithm a couple of variables can be calculated measuring how "clean" the jet is. A clean jet means there is very little noisy data used to reconstruct the jet, and the probability is high that the jet is produced by a cascade of particles.

The jet reconstruction algorithms produce a number of variables that characterize the jets on geometric size, particle content, which detector sub-system received most energy and so on. There are certain combinations of values for these variables for which the probability is very low that the signal in the calorimeter originates from a particle jet. For example if all the energy in the jet is reconstructed only in one sub-system of the detector, it is very likely that this specific piece of hardware was suffering from electronic noise.

The EF algorithms produce additional cleaning related variables for every reconstructed jet, giving a more precise handle on the source of the signal.

\section{Jet cleaning variables}

The jet cleaning variables are part of the output from the jet reconstruction algorithms. They characterize the reconstructed jet in location and time in the detector and its magnitude in size and energy. They also specify which subsystems of the calorimeter detected an energy deposit. Only a limited range is allowed for these values, outside this range the probability of a reconstructed jet being detector noise rises. The different cleaning variables are explained in the next sections. Every single variable can give a good estimate of a jet being a noise, but combining them provides a more powerful tool. This will be detailed after the description of the individual parameters. Per parameter the distribution will be shown in two separate data taking runs from 2012, 203195 and 203934, the former being a particularly noisy run and the latter is used as a cross check.

\section{Jet width using the n90 variable}

The calorimeter of the detector is divided in cells, where each cell does its own energy measurement. The jet is reconstructed using a subset of these cells, and the total energy in the jet is the sum of these measurements. The smallest number of calorimeter cells that contain $90 \%$ of the energy is called the $n 90$ variable of the jet. The $\mathrm{n} 90$ distribution of jets reconstructed by EF in two runs is shown in figure 2.11. If more than $90 \%$ of the energy is found in a single cell, i.e. n90 
$=1$ (see e.g. fig $2.11(\mathrm{a})$ ), it is very likely this particular cell was suffering from electronic noise.

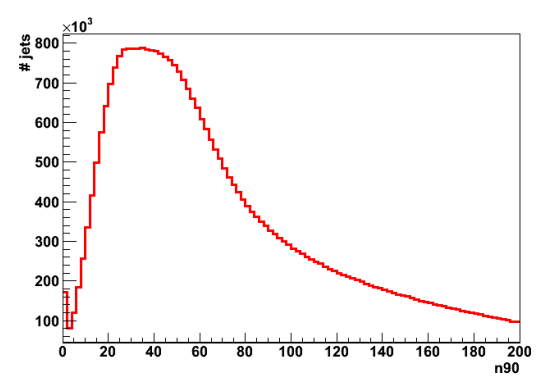

(a) run 203195

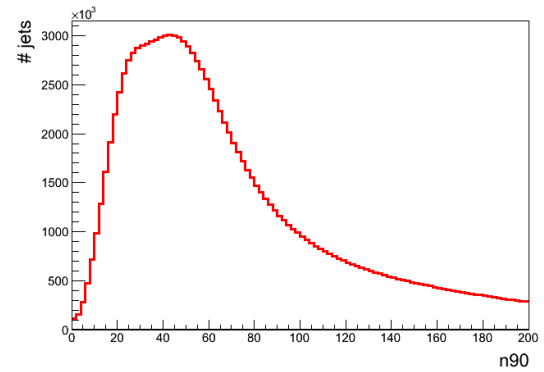

(b) run 203934

Figure 2.11: The n90 distribution of jets reconstructed by EF.

\section{Electronic noise in the presampler: presampler fraction}

The presampler is a thin calorimeter layer which provides the first sampling of the showers in front of the liquid Argon electromagnetic calorimeter. Since this layer is very thin $(11 \mathrm{~mm})$, it is very unlikely that this sub-detector stops the entire shower. If almost all the energy reconstructed in the jet is measured by the presampler it is very likely that the signal came from electronic noise.

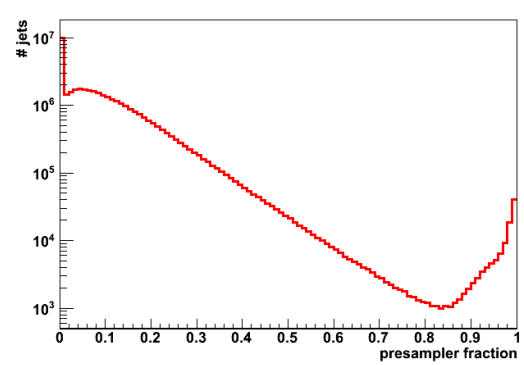

(a) run 203195

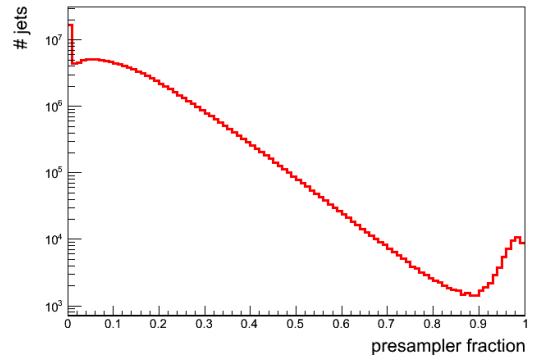

(b) run 203934

Figure 2.12: The presampler fraction distribution of jets reconstructed by EF. 


\section{Calorimeter noise floor: negative energy}

The signal from the cells in the calorimeter has a variable noise floor. From this pedestal the energy measurement of a signal is performed. When the signal is lower than this threshold, a negative energy is obtained. The negative energy of a jet is the energy sum of all cells in the jet that report a negative energy. Figure 2.13 shows the distribution of negative energy in jets reconstructed by the Event Filter.

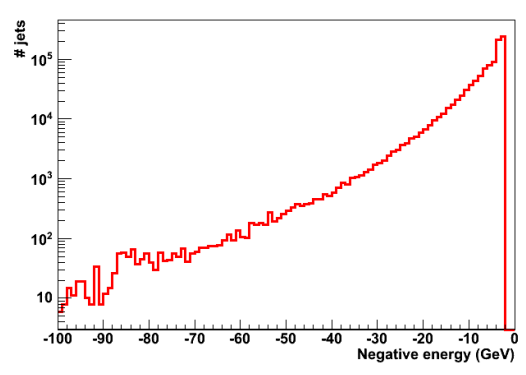

(a) run 203195

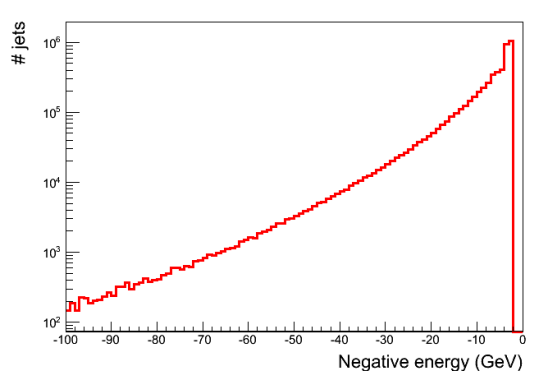

(b) run 203934

Figure 2.13: The negative energy distribution of jets reconstructed by EF.

\section{Jet timing with respect to the bunch crossing}

The cells in the calorimeter time the signal with respect to the bunch crossing. When a jet is reconstructed by the event filter, the average time of the cells in the jet is calculated and taken as the time at which the jet formed with respect to the bunch crossing. When this variable has a high absolute value, let's say $|t|>10$ nanoseconds, the jet is likely reconstructed on noise. Figure 2.14 shows the distribution of the jet timing in jets reconstructed by the Event Filter.

\section{Liquid Argon calorimeter cell signal shape: LAr Quality}

When a particle traverses the active material of a calorimeter cell this cell produces an electrical signal over a small period of time, see figure 2.15 (from [49]). The expected signal shape in the cell is compared to the actual output of the hardware. The result of this comparison is stored as the cell's $Q$ value. This is stored as a 16 bit integer. A very low $\mathrm{Q}$ value means a very good agreement with the model. The jet's LAr Quality is the fraction of cells in the jet that have a $\mathrm{Q}$ value greater than 4000 , i.e. a LAr Quality close to unity means the signals in the cells are likely coming from electronic noise. 


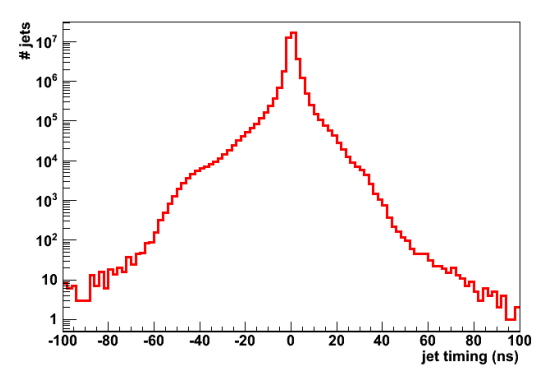

(a) run 203195

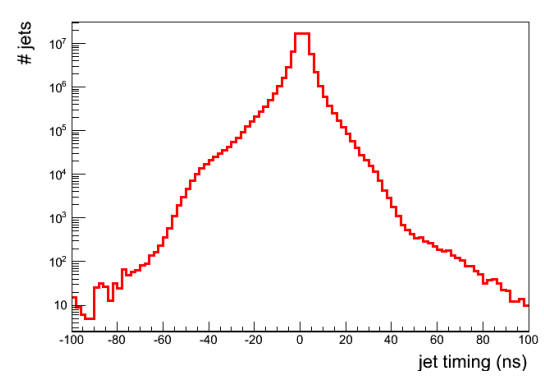

(b) run 203934

Figure 2.14: Jet timing distributions. $\mathrm{t}=0$ is the bunch crossing.

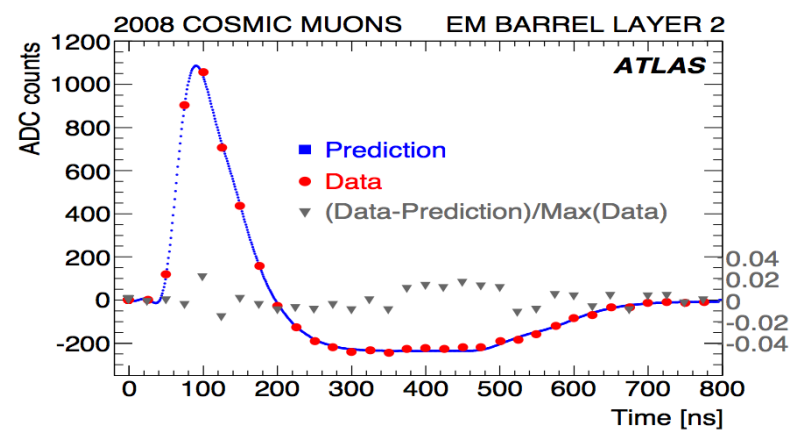

Figure 2.15: The signal produced by a calorimeter cell when a particle traverses the active material.

\section{Fraction of jet energy in end-cap region: HEC fraction}

The HEC fraction is the fraction of energy in the reconstructed jet that is measured by cells located in the Hadronic End Cap Calorimeter. A HEC fraction of zero means all the energy is recorded in the barrel region. Figure 2.17 shows the distribution of the HEC fraction in jets reconstructed by the Event Filter.

\section{Fraction of jet energy in EM calorimeter: EM fraction}

The EM fraction is the fraction of energy in the reconstructed jet that is measured by cells located in the electromagnetic calorimeter. An EM fraction of zero means all the energy in the jet is deposited in the hadronic calorimeter. Figure 2.18 shows the distribution of the EM fraction in jets reconstructed by EF. 


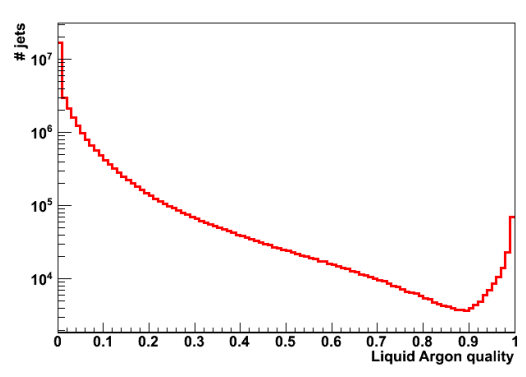

(a) run 203195

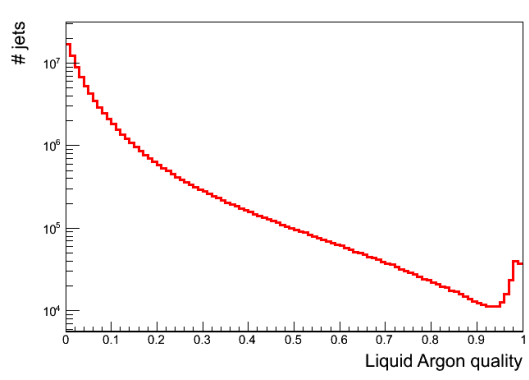

(b) run 203934

Figure 2.16: The Liquid Argon quality distribution of jets reconstructed by EF.

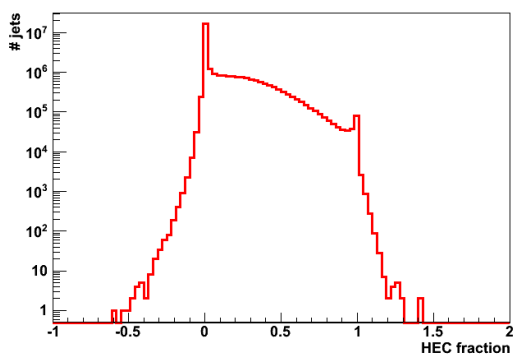

(a) run 203195

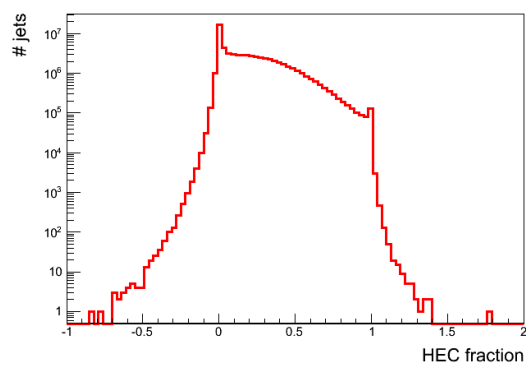

(b) run 203934

Figure 2.17: The HEC fraction distribution of jets reconstructed by EF.

\section{Jet cleaning cuts}

Jet cleaning is already implemented in the offline algorithms, where it flags jets in the output data as valid or invalid. This is an effective method from the jet analysis point of view, but if the offline cuts veto all jets in the event there might have been no need recording it in the first place. This is why it is advantageous to implement the cleaning on-line as well. It is very important though that the jets rejected on-line also would have been rejected by the loosest offline cuts. The offline cuts are outlined in the following section, with the on-line cuts following after that. The performance of the offline cuts on the jets rejected by the on-line cuts is given later in the results section.

The offline cleaning cuts are given in table 2.2. The HECQ variable is the same as the LAr Quality variable, but only measured in the endcap region. LArQmean 


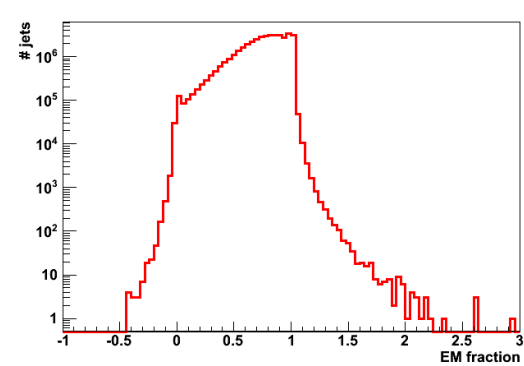

(a) run 203195

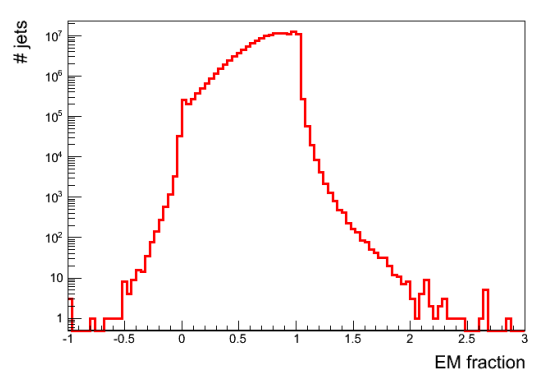

(b) run 203934

Figure 2.18: The EM fraction distribution of jets reconstructed by EF.

\begin{tabular}{|l|l|}
\hline Noise source & $\begin{array}{l}\text { Offline LOOSER cuts 2011 } \\
\text { (also valid for 2012 data) }\end{array}$ \\
\hline \hline HEC spikes & $\begin{array}{c}\text { HECf }>0.5 \text { AND } \mid \text { HECQ } \mid>0.5 \\
\text { AND LArQmean }>0.8\end{array}$ \\
& negative energy $<-60$ GeV \\
\hline EM coherent noise & $\begin{array}{c}\text { EMf }>0.95 \text { AND } \mid \text { LArQ } \mid>0.8 \\
\text { AND LArQmean }>0.8 \\
\text { AND }|\eta|<2.8\end{array}$ \\
\hline $\begin{array}{l}\text { Non-collision } \\
\text { background and } \\
\text { cosmics }\end{array}$ & $\begin{array}{l}\text { EMf }<0.05 \text { AND Chf }<0.05 \text { AND }|\eta|<2 \\
\text { EMf }<0.05 \text { AND }|\eta| \geq 2\end{array}$ \\
\hline
\end{tabular}

Table 2.2: Offline jet cleaning cuts

is the normalized average LAr Quality of the cells in the jet, Chf is the jet charged fraction, defined as the ratio of the scalar sum of the $p_{T}$ of the tracks associated to the jet divided by $p_{T}^{j e t}$, and FMax; the maximum energy fraction in a single calorimeter layer. These variables are not used on-line or in this study. More information on these can be found in the conference note on jet cleaning [50].

The on-line cleaning cuts are given in table 2.3. The online cuts are less complex than the offline cuts to keep them as safe as possible. Measuring the impact of these cuts on data quality and detection efficiency is the goal in this jet study. 


\begin{tabular}{|l|l|}
\hline On-line jet cleaning variables studied & cut value \\
\hline n90 & $<2$ \\
presampler fraction & $>90 \%$ \\
negative energy & $<-60 \mathrm{GeV}$ \\
\hline
\end{tabular}

Table 2.3: On-line jet cleaning cuts

\section{Performance of the jet cleaning}

The jet cleaning is to be implemented in (almost) all the default trigger chains. This means it is very important that the cleaning cuts are not too tight, otherwise valid and valuable data will be discarded and lost forever. This section describes the test procedure and shows the results of the tests, and gives a recommendation on the optimal values for the cuts.

To test the cleaning cuts four chains in the standard menu are duplicated. These duplicates differ from their respective original only in the fact that they have jet cleaning switched on. The duplicates are added to the MC menu. Table 2.4 contains the names of the chains with duplicates where the cleaning is switched on. The suffix _testCleaning is added to the duplicate chains. This new MC

\begin{tabular}{|l|l|}
\hline $\begin{array}{l}\text { Duplicated chains with jet } \\
\text { cleaning switched on }\end{array}$ & type of chain \\
\hline EF_j45_a4tchad_L2FS_L1J15 & low pt chain with L1.5 Hypo \\
EF_j55_a4tchad & low pt chain \\
EF_fj45_a4tchad_L2FS & low pt chain for forward jets \\
EF_j360_a4tchad & high pt chain \\
\hline
\end{tabular}

Table 2.4: Jet trigger chains duplicated to test the jet cleaning

menu is used in the reprocessing of two runs; 203195 and 203934. The former is chosen because it was flagged as having a higher than usual amount of noise, the latter is for comparison and cross check.

Because the MC menu has four identical chains that differ only in the inclusion of the jet cleaning, the ratio of the trigger rates of these chains is a direct measure of the performance of the jet cleaning. Specifically the events are selected which are triggered by the default chain, but not by the _testCleaning duplicate. These events are considered to be noise by the jet cleaning. They are studied to see which cleaning cut rejected the jets, and if they would also be rejected by the offline jet cleaning.

The testing of the jet cleaning is focused on the rate differences between the default and duplicate chains. The next section summarizes these numbers for all cuts together and in the sections after this the cuts on the different variables are 
studied separately.

\section{Trigger rate ratios}

The trigger rates of the chains studied gives the most direct measure of the performance of the jet cleaning. It is the reduction in rate shown by the duplicate chains that will be the effect on operations when activated in the detector. Tables 2.5 for run 203195 and 2.6 for 203934 show the rate reduction caused by the jet cleaning.

\begin{tabular}{|l|l|l|l|}
\hline EF chain & $\begin{array}{l}\text { total } \\
\text { triggers }\end{array}$ & $\begin{array}{l}\text { after } \\
\text { cleaning }\end{array}$ & $\begin{array}{l}\text { cleaning veto's } \\
\text { (\% of total) }\end{array}$ \\
\hline j45_a4tchad_L2FS_L1J15 & 1118510 & 1096958 & $21552(1.9 \%)$ \\
j55_a4tchad & 1025823 & 1004078 & $21745(2.1 \%)$ \\
fj45_a4tchad_L2FS & 6060 & 6045 & $15(0.2 \%)$ \\
j360_a4tchad & 37578 & 37224 & $354(0.9 \%)$ \\
\hline
\end{tabular}

Table 2.5: Events rejected by online jet cleaning cuts in run 203195.

\begin{tabular}{|l|l|l|l|}
\hline EF chain & $\begin{array}{l}\text { total } \\
\text { triggers }\end{array}$ & $\begin{array}{l}\text { after } \\
\text { cleaning }\end{array}$ & $\begin{array}{l}\text { cleaning veto's } \\
\text { (\% of total) }\end{array}$ \\
\hline j45_a4tchad_L2FS_L1J15 & 2676912 & 2648810 & $28102(1.0 \%)$ \\
j55_a4tchad & 2468009 & 2439370 & $28639(1.2 \%)$ \\
fj45_a4tchad_L2FS & 12821 & 12794 & $27(0.2 \%)$ \\
j360_a4tchad & 92562 & 92284 & $278(0.3 \%)$ \\
\hline
\end{tabular}

Table 2.6: Events rejected by online jet cleaning cuts in run 203934.

In low $\mathrm{p}_{T}$ the noisy run 203195 shows a reduction of about $2 \%$, and the cross check run (203934) about $1 \%$. The high $\mathrm{p}_{T}$ chains show a reduction of less than $1 \%$. Figure 2.19 shows the trigger rates per luminosity block. Noise spikes and noisy periods are easy to spot in these plots, as is the effect the jet cleaning has on them. The EF_j55_a4tchad chain is used to generate these figures.

\section{Performance of the n90 cut at L1.5}

The cut on $\mathrm{n} 90$ is the only requirement that is studied in all HLT levels. Therefore it is important to investigate correlation between the values each stage computes. The seed size used in L1.5 is different from L2 and EF, which results in a large difference in the calculated n90 values. L2 and EF use single cells to compute n90, and L1.5 uses trigger towers as the seed for this variable. A trigger tower consist of several cells. This summing of cells has the effect that the value for 


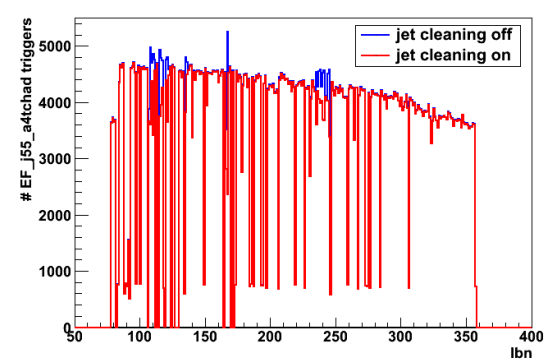

(a) run 203195

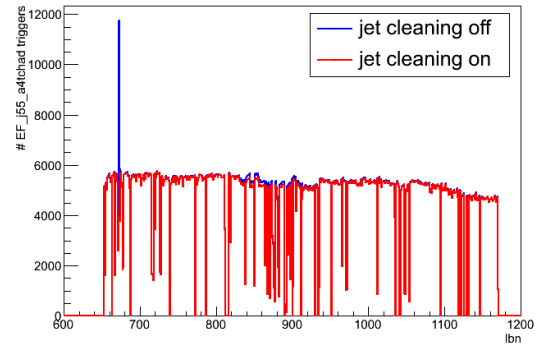

(b) run 203934

Figure 2.19: EF_j55_a4tchad number of events per Luminosity Block (about 1 minute).

$\mathrm{n} 90$ in L1.5 is much lower than what L2 and EF calculate. This can be clearly seen in figure 2.20 .

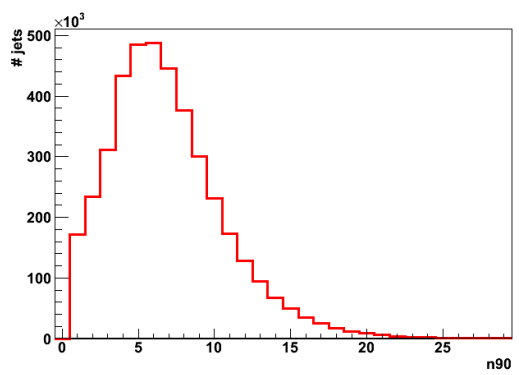

(a) L1.5, run 203195

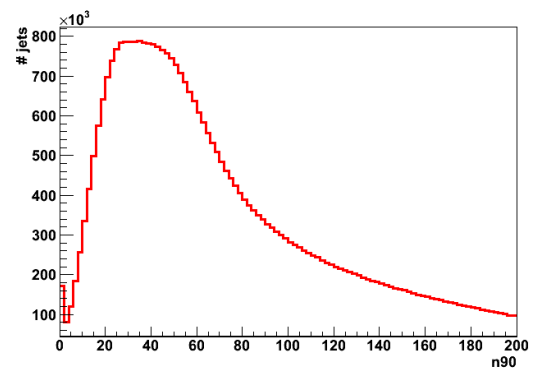

(b) EF, run 203195

Figure 2.20: The n90 distributions of jets reconstructed by L1.5 and EF. Note the range difference on the horizontal axis, due to the different seed values.

From all the jets reconstructed in run 203195 the subset where L1.5 calculated $\mathrm{n} 90(\mathrm{~L} 1.5)=1$ is studied. In particular the value L2 and EF calculated for this variable is examined. Figure 2.21 is the distribution of n90(EF) from jets reconstructed by EF, where L1.5 returned n90(L1.5) $=1$. Distribution 2.21(a) contains 23528 jets with $\mathrm{n} 90(\mathrm{~L} 1.5)=1$. The peak at $\mathrm{n} 90=1$ has 3700 entries, meaning only 3700 out of 23528 jets have also $\mathrm{n} 90(\mathrm{EF})=1$. The remainder of jets, about $84 \%$, would have been rejected by the L1.5 cleaning but not by the EF cleaning. 


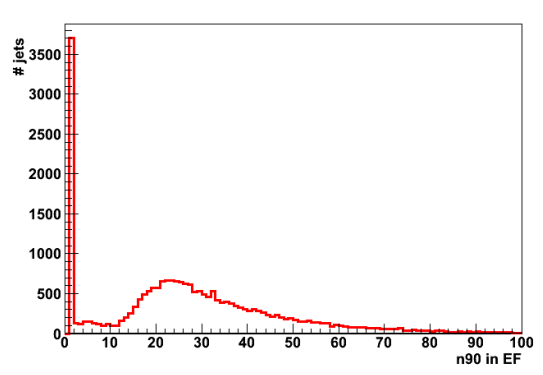

(a) Jet n90(EF) with n90(L1.5)=1.

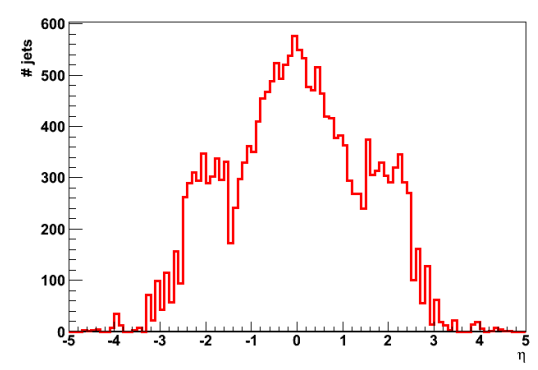

(b) The eta distribution of jets with $\mathrm{n} 90(\mathrm{~L} 1.5)=1$ and $\mathrm{n} 90(\mathrm{EF})>1$.

Figure 2.21: The n90 distribution of jets reconstructed by EF, with n90(L1.5)=1.

Figure 2.21(b) shows the eta distribution of this remaining $84 \%$. This is a cross check to see if there is some eta dependence for these only partially rejected jets, to rule out faulty equipment. It shows no eta dependence at all, suggesting these jets are valid data.

The fact that the n90 values calculated by L1.5 and EF are calculated differently, and that the loosest cut possible for L1.5 is still much tighter than for EF indicates that the $\mathrm{n} 90$ cut should not be applied at L1.5. The n90(L1.5) variable is not considered further in this document, and for readability every following mention of $n 90$ refers to $n 90(\mathrm{EF})$.

\section{Performance of the n90 cut at EF}

Tables 2.7 and 2.8 show the rejection rate and false rejection rate of the $n 90<2$ cut in the studied runs. A false rejection is a rejection by the online cuts that would have passed the offline cuts. The chain names in the tables are abbreviated from now on to improve readability.

For low $\mathrm{p}_{T}$ jets averaged over both runs the $\mathrm{n} 90$ cut is responsible for more than $90 \%$ of the rejected events, while it is responsible for only about $5 \%$ of the false rejections. It appears that the $\mathrm{n} 90$ cut is the workhorse of the jet cleaning while making only few errors.

\section{Performance of the presampler fraction cut}

Tables 2.9 and 2.10 show the rejection rate and false rejection rate of the presampler fraction $>90 \%$ cut in the studied runs. The rejection rate show a similar but slightly smaller number as for the $\mathrm{n} 90$ cut, but the number of false rejections 
shows that this cut is responsible for almost all errors.

The value of the cut is studied further to check if it can be improved upon to an acceptable level. The value is increased to find if there is an optimal cut that has little false rejections. Figure 2.22 shows the ratio of the false rejections to total rejections when the value of the cut is increased.

The false / total rejection ratio is not improved by increasing the cut value. Together with the fact that this cut has a lot of overlap with the n90 cut in event rejection the result suggests to leave this cut out.

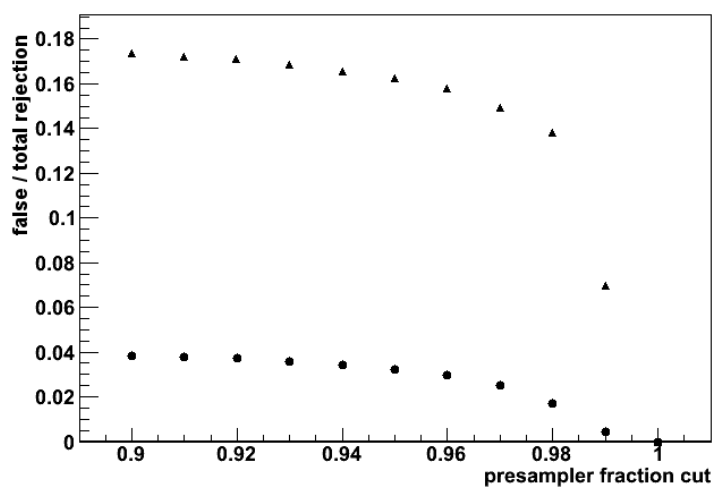

Figure 2.22: Ratio false / total / rejections by the presampler fraction cut. Circles are run 203195 and triangles run 203934. Increasing the cut value does not improve the false / total rejection ratio, except for values close to 1 where the cut itself has no effect anymore.

\section{Performance of the negative energy cut}

Tables 2.11 and 2.12 show the rejection rate and false rejection rate of the negative energy $<-60 \mathrm{GeV}$ cut in the studied runs. The impact of this cut is very low. However, it also does not have false rejections so it is save to include since it has some effect in the forward and high $\mathrm{p}_{T}$ chains.

\section{Conclusion}

Basic jet cleaning with cuts on n90, presampler fraction and negative energy is studied in the HLT levels L1.5, L2 and EF. The n90 cut in L2 and EF is found to be the most effective cut in this study, responsible for about $90 \%$ of all rejected 
events while having little false rejections. The n90 cut should not be implemented at L1.5 however because the difference in granularity of the seed at this level.

The presampler fraction cut at EF is responsible for almost all the false rejections of the jet cleaning. Considering it rejects many events that the n90 cut also rejects, this study suggests that this variable is not suitable to use as a cleaning variable in the Event Filter.

The negative energy cut does very little in rate reduction, and had only one false rejection in both runs under study. Since it has some effect (1-2\% in rate) in the high $\mathrm{p}_{T}$ and forward chains this study suggests this cut can be included at Event Filter. 


\begin{tabular}{|l|l|l|l|l|}
\hline chain & $\begin{array}{l}\text { cleaning } \\
\text { veto's }\end{array}$ & veto's n90 cut & $\begin{array}{l}\text { false veto's } \\
\text { all cuts }\end{array}$ & $\begin{array}{l}\text { false veto's } \\
\text { n90 cut }\end{array}$ \\
\hline EF_j45 & 21552 & $20614(95.6 \%)$ & $795(3.7 \%)$ & $51(6.4 \%)$ \\
EF_j55 & 21745 & $20665(95.0 \%)$ & $793(3.6 \%)$ & $35(4.4 \%)$ \\
EF_fj45 & 15 & $13(86.7 \%)$ & $0(0.0 \%)$ & $0(0.0 \%)$ \\
EF_j360 & 354 & $314(88.7 \%)$ & $24(6.8 \%)$ & $0(0.0 \%)$ \\
\hline
\end{tabular}

Table 2.7: Rejection rate and false rejections of the online n90 cleaning in run 203195. The percentages given after the counts are the fraction of total number of veto's in column 3 and 4, and in the last column the fraction of total number of false rejections.

\begin{tabular}{|l|l|l|l|l|}
\hline chain & $\begin{array}{l}\text { cleaning } \\
\text { veto's }\end{array}$ & veto's n90 cut & $\begin{array}{l}\text { false veto's } \\
\text { all cuts }\end{array}$ & $\begin{array}{l}\text { false veto's } \\
\text { n90 cut }\end{array}$ \\
\hline EF_j45 & 28102 & $24478(87.1 \%)$ & $2925(10.4 \%)$ & $151(5.2 \%)$ \\
EF_j55 & 28639 & $24592(85.9 \%)$ & $2946(10.3 \%)$ & $127(4.3 \%)$ \\
EF_fj45 & 27 & $24(88.9 \%)$ & $0(0.0 \%)$ & $0(0.0 \%)$ \\
EF_j360 & 278 & $117(42.1 \%)$ & $117(42.1)$ & $0(0.0 \%)$ \\
\hline
\end{tabular}

Table 2.8: Rejection rate and false rejections of the online n90 cleaning in run 203934. The percentages given after the counts are the fraction of total number of veto's in column 3 and 4, and in the last column the fraction of total number of false rejections.

\begin{tabular}{|l|l|l|l|l|}
\hline chain & $\begin{array}{l}\text { cleaning } \\
\text { veto's }\end{array}$ & $\begin{array}{l}\text { veto's presampler } \\
\text { fraction cut }\end{array}$ & $\begin{array}{l}\text { false veto's } \\
\text { all cuts }\end{array}$ & $\begin{array}{l}\text { false veto's } \\
\text { presampler } \\
\text { fraction cut }\end{array}$ \\
\hline EF_j45 & 21552 & $19729(91.5 \%)$ & $795(3.7 \%)$ & $768(96.6 \%)$ \\
EF_j55 & 21745 & $19768(90.9 \%)$ & $793(3.6 \%)$ & $754(95.1 \%)$ \\
EF_fj45 & 15 & $0(0.0 \%)$ & $0(0.0 \%)$ & $0(0.0 \%)$ \\
EF_j360 & 354 & $257(72.6 \%)$ & $24(6.8 \%)$ & $24(100.0 \%)$ \\
\hline
\end{tabular}

Table 2.9: Rejection rate and false rejections of the online presampler fraction cleaning in run 203195. The percentages given after the counts are the fraction of total number of veto's in column 3 and 4, and in the last column the fraction of total number of false rejections. 


\begin{tabular}{|l|l|l|l|l|}
\hline chain & $\begin{array}{l}\text { cleaning } \\
\text { veto's }\end{array}$ & $\begin{array}{l}\text { veto's presampler } \\
\text { fraction cut }\end{array}$ & $\begin{array}{l}\text { false veto's } \\
\text { all cuts }\end{array}$ & $\begin{array}{l}\text { false veto's } \\
\text { presampler } \\
\text { fraction cut }\end{array}$ \\
\hline EF_j45 & 28102 & $16161(57.5 \%)$ & $2925(10.4 \%)$ & $2823(96.5 \%)$ \\
EF_j55 & 28639 & $16252(56.7 \%)$ & $2946(10.3 \%)$ & $2824(95.9 \%)$ \\
EF_fj45 & 27 & $0(0.0 \%)$ & $0(0.0 \%)$ & $0(0.0 \%)$ \\
EF_j360 & 278 & $229(82.4 \%)$ & $117(42.1 \%)$ & $117(100.0 \%)$ \\
\hline
\end{tabular}

Table 2.10: Rejection rate and false rejections of the online presampler fraction cleaning in run 203934. The percentages given after the counts are the fraction of total number of veto's in column 3 and 4 , and in the last column the fraction of total number of false rejections.

\begin{tabular}{|l|l|l|l|l|}
\hline chain & $\begin{array}{l}\text { cleaning } \\
\text { veto's }\end{array}$ & $\begin{array}{l}\text { veto's negative } \\
\text { energy cut }\end{array}$ & $\begin{array}{l}\text { false veto's } \\
\text { all cuts }\end{array}$ & $\begin{array}{l}\text { false veto's } \\
\text { negative } \\
\text { energy cut }\end{array}$ \\
\hline EF_j45 & 21552 & $39(0.2 \%)$ & $795(3.7 \%)$ & $0(0.0 \%)$ \\
EF_j55 & 21745 & $40(0.2 \%)$ & $793(3.6 \%)$ & $0(0.0 \%)$ \\
EF_fj45 & 15 & $2(13.3 \%)$ & $0(0.0 \%)$ & $0(0.0 \%)$ \\
EF_j360 & 354 & $5(1.4 \%)$ & $24(6.8 \%)$ & $0(0.0 \%)$ \\
\hline
\end{tabular}

Table 2.11: Rejection rate and false rejections of the online negative energy cleaning in run 203195. The percentages given after the counts are the fraction of total number of veto's in column 3 and 4 , and in the last column the fraction of total number of false rejections.

\begin{tabular}{|l|l|l|l|l|}
\hline chain & $\begin{array}{l}\text { cleaning } \\
\text { veto's }\end{array}$ & $\begin{array}{l}\text { veto's negative } \\
\text { energy cut }\end{array}$ & $\begin{array}{l}\text { false veto's } \\
\text { all cuts }\end{array}$ & $\begin{array}{l}\text { false veto's } \\
\text { negative } \\
\text { energy cut }\end{array}$ \\
\hline EF_j45 & 28102 & $41(0.1 \%)$ & $2925(10.4 \%)$ & $0(0.0 \%)$ \\
EF_j55 & 28639 & $40(0.1 \%)$ & $2946(10.3 \%)$ & $1(0.0 \%)$ \\
EF_fj45 & 27 & $3(11.1 \%)$ & $0(0.0 \%)$ & $0(0.0 \%)$ \\
EF_j360 & 278 & $5(1.8 \%)$ & $117(42.1 \%)$ & $0(0.0 \%)$ \\
\hline
\end{tabular}

Table 2.12: Rejection rate and false rejections of the online negative energy cleaning in run 203934. The percentages given after the counts are the fraction of total number of veto's in column 3 and 4 , and in the last column the fraction of total number of false rejections. 


\section{DATA PREPARATION FOR W BOSON PAIR PRODUCTION IN DPI EVENTS}

With the ever increasing center of mass energies and luminosities in the modern colliders Double Parton Interactions are becoming more accessible then ever for physics studies. On the other hand they are also forming a more and more important background for the new particle searches. It is therefore very important to study its behavior and magnitude. The first question after determining the cross section is: Can the process be approximated by two independent interactions, or do they interfere with one another? To answer these questions it is important to look at clean production events. DPI with jets in the final state are the most common, but the jets mean that the underlying process cannot be determined. The type of DPI events with highest cross section and clear underlying process is $\mathrm{W}$ pair production, where the $\mathrm{W}$ decays to a charged lepton and a neutrino. However, W pairs are produced copiously in the tree-level diagram in figure 3.1. Fortunately these $\mathrm{W}$ pairs are always produced with opposite charge, and can easily be filtered out by adding a same charge constraint to the event selection. In fact, having two (no more, no less) high energy leptons of the same charge is a very rare final state, and the DPI contribution is by far the largest in this event selection. In section 3.1 the simulation of DPI events is discussed. Section 3.2 describes the event topology that we have been looking for in the detector that are characteristic for $\mathrm{W}$ pair production in DPI events. Unfortunately due to detector efficiencies there is still a large background present in the event selection. This is discussed in section 3.3. 

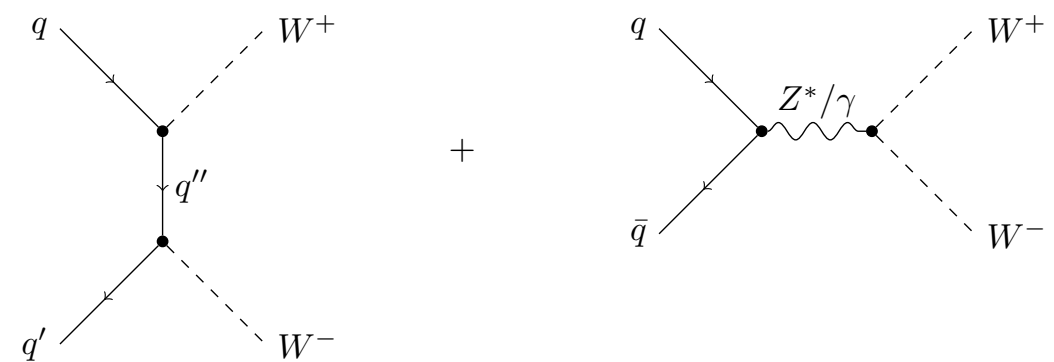

Figure 3.1: Opposite sign W pair production at tree level. This background process is effectively eliminated by requiring same sign final state leptons in the event.

\subsection{Simulation}

Double parton interactions are part of the underlying event (UE), together with initial and final state radiation (ISR,FSR) and beam remnants among others. So in order to studying DPI events we need to know the relation between the constituents of the UE. Currently there are two main approaches commonly used to simulate DPI events, with the main purpose of determining how many interactions there are in the UE. The process implemented in Pythia uses a $p_{T}$ ordered Sudakov [51] formalism and more extensive corrections like color reconnection and rescattering, where the conservation laws are required at each step. An overview of the most important model parameters is given in section 3.1.1. The method implemented in Herwig $++[52]$ uses a matter distribution related to the electromagnetic from factor where the number of MPI is computed using Poisson statistics, including vetoes if energy or momentum conservation is violated. Section 3.1.2 describes this procedure in more detail. In section 3.1.3 both generators are compared. To construct two data sets that compare these two approaches both applications are run using their default parameters. This is in contrast with the DPI signal data set used in the main analysis, where the Pythia event generator is used with the ATLAS 2012 parameter tune.

\subsubsection{DPI simulation using Pythia}

The most naive approximation is a simple factorization; use Poisson statistics to determine the probability of a subsequent hard scatter next to the primary interaction and add that to the underlying event. However, this approach is too crude. Pythia employs various techniques to refine this approach. Basically, it generates a list of partonic scatters ordered in $p_{T}$, starting at the primary 
interaction. The probability of the QCD interactions to branch is determined by the Sudakov form factor, and every new branch is added to the $p_{T}$ ordered list. In every step vetoes are in place to conserve energy and momentum. Both initial state radiation and MPI is evolved in one common sequence, interleaving the QCD UE. In Pythia, the second (MPI) scatter can be chosen at will, making it very suitable and efficient to study double parton interactions.

The correlations in momentum and flavor are included in the model by the application of interleaved evolution [53]. As a starting point, the standard parton density functions are taken in constructing parton distributions for the remnant hadronic object after one or several interactions have occurred. To conserve momentum and flavor a couple adjustments are introduced. The first and most trivial is the removal of the momentum fraction $x$ removed by the previous interaction(s). The parton densities are assumed to scale such that the point $x=1$ corresponds to the remaining momentum, instead of the full momentum of the initial proton. The second modification is on the normalization of the quark distributions. If a valence quark is knocked out in a previous scatter, the number of remaining quarks is reduced accordingly. Similarly when a sea quark is removed, it leaves behind an extra anti sea quark available for the subsequent interactions. These two main modifications together with conservation of transverse momenta of the partons and the longitudinal momenta of the beam remnants considerably refines the crude approach of simple factorization. These conservation laws are all imposed in every step in the ordered $p_{T}$ sequence, which contains both ISR and MPI contributions. This model effectively creates a dependence between all components of the underlying event.

\subsubsection{DPI simulation using Herwig ++}

The dijet production inclusive cross section $\sigma^{i n c}\left(s ; p_{t}^{\min }\right)$ will exceed the total cross section for high enough energies.

$$
\sigma^{i n c}\left(s ; p_{t}^{m i n}\right)=\sum_{i, j} \int_{\left(p_{t}^{m i n}\right)^{2}} d p_{t}^{2} f_{i / h_{1}}\left(x_{1}, \mu^{2}\right) \times \frac{d \hat{\sigma}_{i, j}}{d p_{t}^{2}} \times f_{j / h_{2}}\left(x_{2}, \mu^{2}\right)
$$

This excess is interpreted as the inclusive cross section not only counting single hard events, but all hard scatters that occur simultaneously in the same protonproton collision. So at a certain high energy $E$ where $\sigma^{i n c} / \sigma^{t o t}=1.5$, there is a $50 \%$ chance of having a second hard scatter. This inference is used in Herwig ++ to obtain the number of MPI interactions in a simulated event. The average number of hard interactions is expressed as

$$
\bar{n}(b, s)=A\left(b ; \mu^{2}\right) \sigma^{i n c}\left(s ; p_{t}^{\text {min }}\right)
$$


where the function $A\left(b ; \mu^{2}\right)$ describes the overlap of the two colliding protons, modeled according to the electromagnetic form factor [54]. The parameter $\mu$ is interpreted as the inverse of the proton radius. With this model it is possible to simulate events with perturbative hard interactions with $p_{T}>p_{T}^{\min }$. The lower momentum interactions are modeled in a similar way, but with parameters $\sigma_{\text {soft }}^{\text {inc }}$ and $\mu_{\text {soft }}^{2}$. After determination of the average number of MPI a color reconnection scheme is applied on the QCD clusters in the event, which is tuned to ATLAS minimum bias data.

\subsubsection{Comparison between Pythia and Herwig ++}

To compare the two different models several distributions of important variables are analyzed. In this exploratory study both simulation programs are run with all parameters set to their default values, creating only $\mathrm{W}$ bosons which decay leptonically in both the first and second hard scatter event. First a set of baseline cuts is used as input for the simulation, see table 3.1, and a couple additional cuts is applied on the resulting data sets, as given in table 3.2. Cuts related to jet

\begin{tabular}{|r||l|l|l|l|l|}
\hline variable: & \# leptons & flavor & $p_{T}$ & $\eta$ & $E_{T}^{\text {miss }}$ \\
\hline value: & 2 & $\mathrm{e}, \mu$ & $>10 \mathrm{GeV}$ & $<5$ & $>10 \mathrm{GeV}$ \\
\hline
\end{tabular}

Table 3.1: MC generator cuts for Pythia and Herwig++.

reconstruction are applied to the output data sets of the event generators. These cannot be imposed on the generators itself because the jet finding algorithm runs separately from the event generation process. The jets are reconstructed by the anti- $k_{T}$ algorithm with a radius of 0.4 in $\eta, \phi$ space. The $\Delta \mathrm{R}$ cut between jets en electrons is to prevent electrons produced in the jet as being flagged as prompt electrons from an initial $\mathrm{W}$ decay, adding a false lepton to the event lepton count.

\begin{tabular}{|r||l|l|l|l|l|l|}
\hline variable: & lepton $p_{T}$ & $\# \mathrm{j}$ & $E_{T}^{\text {jet }}$ & jet $\eta$ & j-e $\Delta \mathrm{R}$ & misc. \\
\hline value: & $>20 \mathrm{GeV}$ & $<2$ & $>25 \mathrm{GeV}$ & $<5$ & $>0.3$ & ssW \\
\hline
\end{tabular}

Table 3.2: Analysis cuts applied on the outputs of the event generators.

First the characteristics of the measurable quantities are compared, like the transverse momenta of the muons and the missing transverse momentum. Figures 3.2 and 3.3 show high compatibility between the two DPI models described above, as far as the measurable variables are concerned. The single exception is in the distributions of the leading lepton transverse momenta. Pythia has slightly less event in the top of the peak, which are found in the start of the tail. They have 
about $10 \mathrm{GeV}$ more transverse momentum compared to the Herwig ++ muons. This difference originates in the slight difference in $\mathrm{W}$ boson transverse momenta seen in figure 3.4. The distribution of the leading lepton transverse momenta and the $E_{T}^{\text {miss }}$ show striking similarity between the single $\mathrm{W}$ boson distributions given in [55]. This is an important indication of the feasibility to reconstruct both $\mathrm{W}$ bosons separately later in this study.
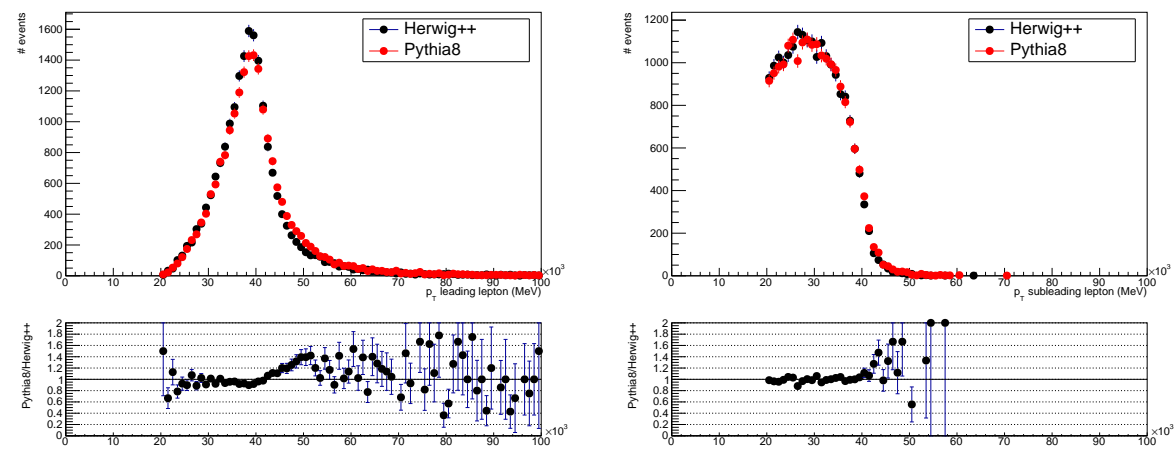

Figure 3.2: Muon transverse momenta, left the leading muon distribution and right the subleading muon. The event generators give highly compatible results in the entire energy range, except for a small deviation around $50 \mathrm{GeV}$ for the leading lepton. Pythia has slightly less events in the top of the peak, which are found in the start of the tail with about $10 \mathrm{GeV}$ more transverse energy. This is due to the difference between the modeling of the $\mathrm{W}$ boson transverse momenta by the two event generators as seen in figure 3.4.

Next we also compare quantities that are not measurable in the detector, like the momenta of the W's. Figure 3.4 indicates that the two models are fairly comparable, except that Herwig ++ produces more low $p_{T} \mathrm{~W}$ bosons, while Pythia tends to give a bit more transverse momentum to the W's. However in figures 3.2 and 3.3 the direct observables seem not to be significantly influenced by this, so this effect is regarded as a consequence of the inner workings of the specific event generators. Another important conclusion from the simulation is the angle between the muon and the neutrino after the $\mathrm{W}$ decays as shown in figure 3.5 . It is crucial to note that the vast majority of W's have their decay products traversing the detector anti-parallel, or back-to-back. In the study of the event topology this will come back to construct a derived variable that tries to reconstruct the initial $\mathrm{W}$ from the decay products. Since there are two charged lepton tracks and only one direction variable for both neutrinos combined $\left(E_{T}^{\text {miss }}\right)$, this back-to-back relation can be used to reconstruct the two neutrino directions. 

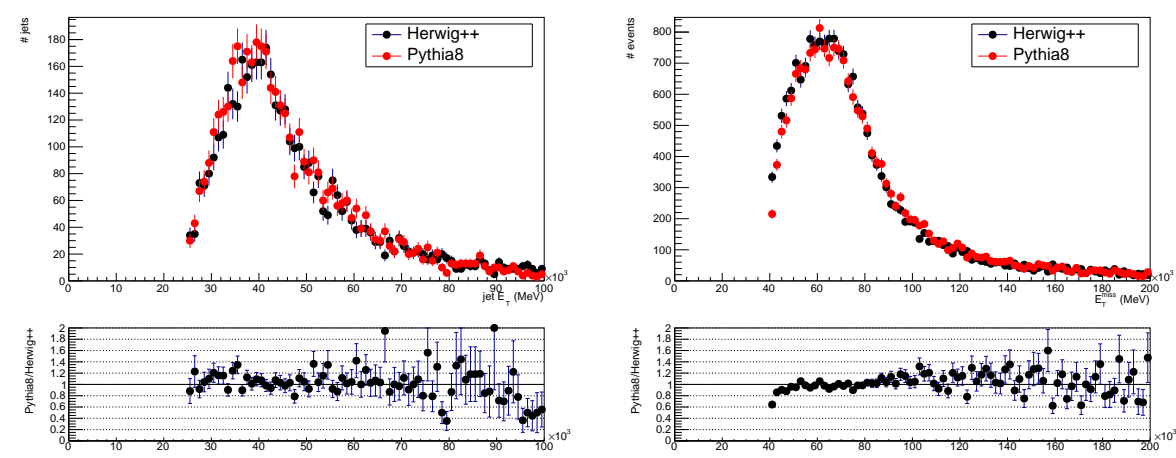

Figure 3.3: Jet $E_{T}$ on the left and $E_{T}^{\text {miss }}$ on the right. Only one high $p_{T}$ jet is allowed in the event, but not every event has a jet that passed this cut. Both event generators are fully compatible within the (statistical) error margins. The $E_{T}^{\text {miss }}$ distribution also shows high compatibility. The events used in this study are in the range $30 \mathrm{GeV}<E_{T}^{\text {miss }}<70 \mathrm{GeV}$.
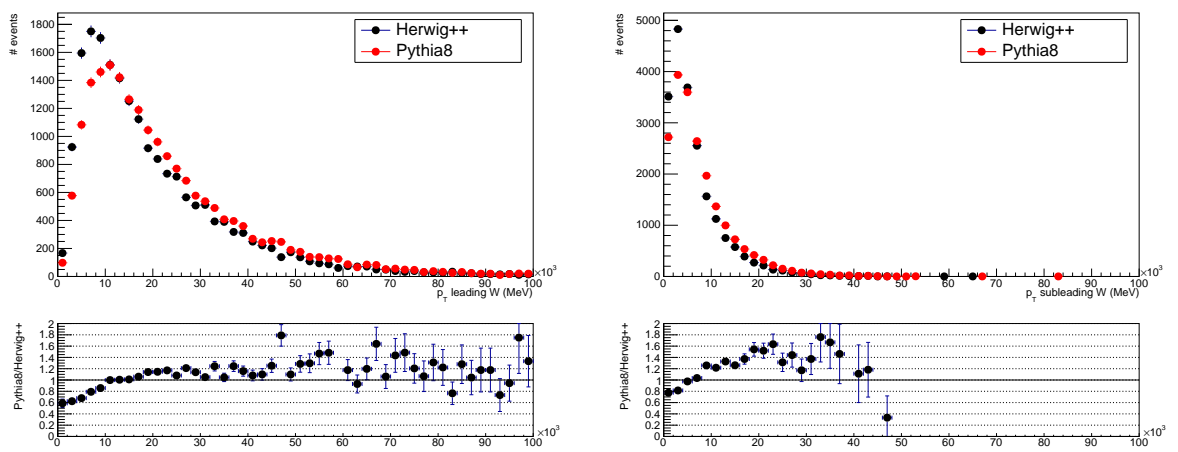

Figure 3.4: W boson momenta distributions, left for leading and right subleading $\mathrm{W}$. The Pythia event generator gives the $\mathrm{W}$ bosons a bit more transverse momentum compared to Herwig ++ . This effect almost vanishes for the direct observables shown in figures 3.2 and 3.3.

\subsection{Signal: Event topology}

In our analysis we are only interested in three basic measurables from the detector. These are the momentum of the highest energy (leading) muon $p_{T}^{\mu 1}$, the momentum of the other (subleading) muon $p_{T}^{\mu 2}$ and the missing transverse mo- 

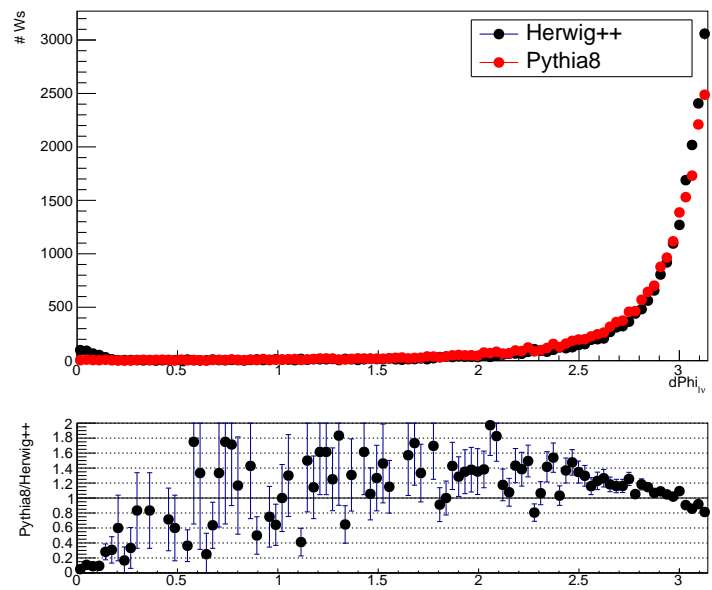

Figure 3.5: The angle between the muon and the neutrino in $\mathrm{W}$ decays. This variable is not directly observable in the data, but has important implications. The distribution shows that the muon and the neutrino have high probability of being produced back-to-back in the detector, which gives an important handle on the neutrino direction in this study.

mentum $E_{T}^{\text {miss }}$ in the calorimeter. From these variables we try to reconstruct the production of two $\mathrm{W}$ bosons in one single proton-proton collision. One main problem that immediately comes to mind is the fact that we have two neutrino's that are measured in one single variable: the missing transverse momentum. The momenta of both neutrino's have to be deduced from this one quantity. In the simulations in section 3.1 figure 3.5 we have seen that most W's decay with the muon and the neutrino almost back-to-back. This greatly simplifies our decomposition of the missing transverse momentum into two neutrino momenta, since we already have an approximation of their direction. If we further assume that the bulk of the missing transverse momentum is coming from the two neutrinos from the $\mathrm{W}$ decay, also the magnitude of the neutrino momentum is known. This procedure is shown in figure 3.6.

Three event variables are measured by the detector: the $p_{T}^{\mu 1}, p_{T}^{\mu 2}$ and $E_{T}^{m i s s}$. Next the vector sum of the transverse momenta $p_{T}^{\mu \mu}$ is calculated. On this vector the missing transverse momentum is projected, giving the $E_{T}^{\text {miss }}$ contribution from the two neutrinos if they were produced back-to-back with respect to the muons, $E_{T}^{m i s s, \mu \mu}$. Finally this $E_{T}^{m i s s, \mu \mu}$ is decomposed in $p_{T}^{\nu 1}$ and $p_{T}^{\nu 2}$ using $p_{T}^{\mu 1}$ and $p_{T}^{\mu 2}$ as basis. 


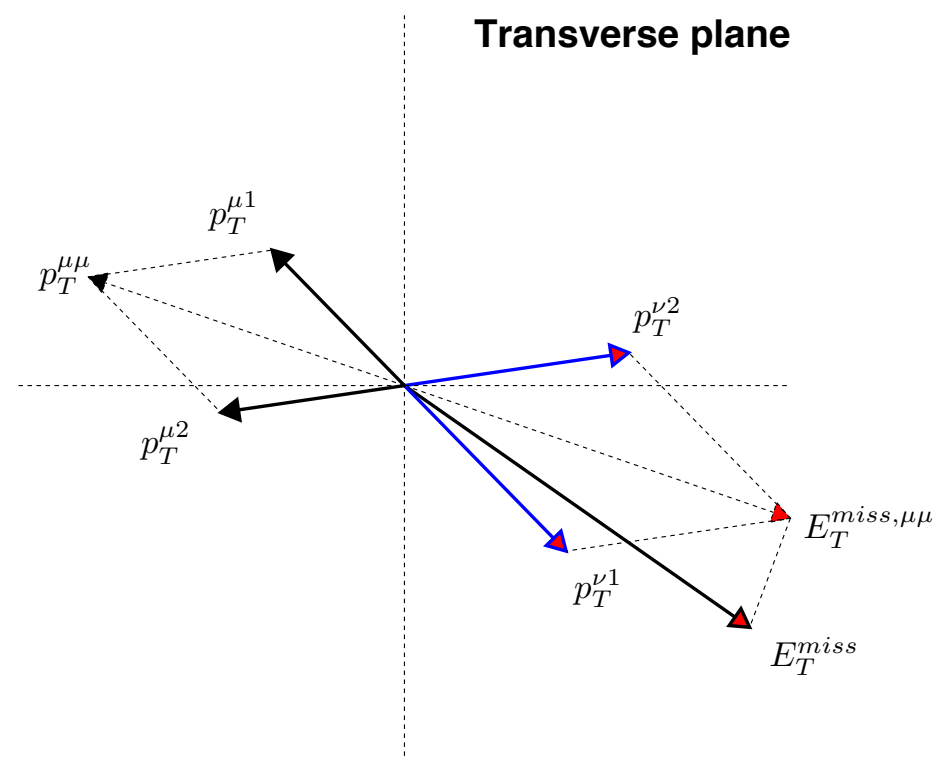

Figure 3.6: Event topology. $p_{T}^{\mu 1}, p_{T}^{\mu 2}$ and $E_{T}^{m i s s}$ are measured by the detector. $p_{T}^{\mu \mu}$ is calculated as the vector sum of $p_{T}^{\mu 1}$ and $p_{T}^{\mu 2}$, after which $E_{T}^{m i s s, \mu \mu}$ is determined as the projection of $E_{T}^{m i s s}$ on $p_{T}^{\mu \mu}$. Finally $E_{T}^{m i s s, \mu \mu}$ is decomposed in $p_{T}^{\nu 1}$ and $p_{T}^{\nu 2}$ using $p_{T}^{\mu 1}$ and $p_{T}^{\mu 2}$ as basis.

With both muon momenta estimated, the transverse momentum of the assumed W boson can now be calculated. In this study this variable is referred to as the transverse $\mathrm{W}$ reconstruction quality $\left(W Q_{T}\right)$, and is calculated with equation 3.3 .

$$
W Q_{T}=\sqrt{4 p_{T}^{\mu} p_{T}^{\nu}}
$$

Usually there is a cosine term in the calculation of a transverse mass, but since the decay products are assumed to be back-to-back the equation is a simple multiplication of momenta. A factor 4 is added to move the value close to the rest mass of a $\mathrm{W}$ boson. Of course there is a certain error on this estimation, but since we are not interested in the exact value of the momenta this can be neglected. The main goal of this decomposition is to create a variable that may give a hint to the structure of the event under study; is it a W pair from DPI or a background process? 


\subsection{Noise: Processes with similar topology}

Two muons (no more, no less) with the same charge in the final state is extremely rare. This would provide a very clean signal for same sign $\mathrm{W}$ pair production, however due to detector efficiencies several background processes contaminate the data set. There are processes that produce a single muon and a fake muon, where the reconstruction algorithms wrongly identified a different object as a prompt muon. The biggest contribution in this class is the $W+$ jets process. This background is notoriously difficult to model, since the simulation program can not be instructed to generate a jet that will be misidentified as a muon in the detector. Section 3.3.1 explains in more detail how this background class is taken into account.

Another class of background processes contains three muons in the final state, where the detector fails to find the third. The main contribution here comes from $W+\gamma^{*} / Z$. This process is quite well understood, and multiple event generators exist to create Monte Carlo sets. The process description and its generators are discussed in section 3.3.2.

A third but smaller contribution comes from processes with four muons in the final state, where the detector misses two of them. These are primarily $Z Z$ events, see section 3.3.3.

\subsubsection{Single muon + fake muon final state: $\mathrm{W}+$ jets}

Background processes in the fake muon class are basically all interactions that produce a single muon, and account for a large part of the background events in the data set. The fake muon is a muon produced in a jet mostly from heavy flavor decay. The main contribution comes from $\mathrm{W}$ production in combination with a jet, but other processes like top quark production are also present. Since there are many processes covered in this background combined with the difficulty of correctly simulate the $\mathrm{W}+$ jets process with a false muon the total contribution of this class is approximated using a data driven approach. The data set for this background class is filled from the detector data set, using the event selection criteria as for the signal data set, except for the subleading muon. The cuts for the subleading muon are such as to optimize the probability of this lepton being a fake lepton by loosening the isolation criteria. It is found, see section 4.1.1, that the shapes of the variable distributions are comparable between this control region and the signal region. The only thing left to do is the normalization.

\section{isolation criteria}

The muon selection criteria involve parameters that give a measure of how much activity there was in the detector directly around the path of the muon. If there 
is little activity, the muon is probably coming directly from the initial process. When there is a lot of energy detected around its path, the muon is likely produced in a jet.

The event selection criteria that were changed to produce the fake muon data set are given in table 3.3, whereas a full description of the signal cuts are given in section 3.4. First the trigger requirement is changed from an isolated muon trigger EF_mu24i_tight to its non-isolated counterpart EF_mu24_tight. Two additional cuts, one on separation in $\phi$ space of the highest $p_{T}$ and jet and one on $M_{T}=\sqrt{2 p_{T}^{\ell} E_{T}^{\text {miss }}\left(1-\cos \left(\phi_{E_{T}^{m i s s}}-\phi_{\ell}\right)\right)}$ are applied, to enrich the sample with $\mathrm{W}+$ jet and dijet events. Several supplementary isolation based cuts from the event selection are removed to enhance the fake muon events to pass the veto. The $z_{0}$ and $d_{0}$ variables are a measure of how far the muon is created from the primary vertex, and the $E_{T}^{i s o}$ and $p_{T}^{i s o}$ variables are an indication of the amount of energy deposited in the calorimeter in the vicinity of the reconstructed track of the muon.

\begin{tabular}{|r||l|l|l|l|}
\hline var: & trigger & $\Delta \phi \mathrm{j}-\mu$ & $M_{T}$ & removed cuts \\
\hline value: & mu24_tight & $>2.8$ & $<40 \mathrm{GeV}$ & $z_{0}, d_{0}, E_{T}^{\text {iso }}, p_{T}^{i s o}$ \\
\hline
\end{tabular}

Table 3.3: Selection criteria on isolation for fake muons.

By removing the isolation criteria in the event selection on the subleading muon a data set is constructed mainly consisting of events with a signal muon and a fake muon. The distributions of this data set are similar to the distributions of the events that have one fake muon where the fake muon passes the signal cuts (and ends up in the signal data set). This is shown in section 4.1.1 where the distributions of the other background classes are subtracted from the data set with detector data. After subtraction the remaining distributions should be from the fake muon class, which is proven to be a valid assumption.

\section{other final states with fake muons}

The $\mathrm{W}+$ jets process is not the only type of events that produces one prompt and one fake muon. Other processes are for example single top production, where the top quark decays via a $\mathrm{W}$ which in turn produces a fake muon. Also $t \bar{t}$ and $b \bar{b}$ production processes are contributing, where the heavy flavor decays via a $\mathrm{W}$, which in turn decays leptonically. These contributions to the fake background class are smaller than the $\mathrm{W}+$ jets process, and since the selection criteria for the data set for this class are not optimized to the $\mathrm{W}+$ jets events, these processes are also taken into account in the procedure described earlier. The results of the validation presented in section 4.1 .1 verify this assumption. 


\subsubsection{Three muon final state: $\mathbf{W}+\gamma^{*} / \mathrm{Z}$}

The $\mathrm{W}+\gamma^{*} / \mathrm{Z}$ process accounts for most of the remainder of the background events in the data set. This background class is also a consequence of detection inefficiency, mainly because the difficulty of instrumenting the area around the beam pipe. This process passes the event selection cuts when both the $\mathrm{W}$ and the Drell Yan (DY) boson decay to muons, and the muon from the DY boson that has opposite charge of the $\mathrm{W}$ escapes the detector unseen. This escape usually happens through an uninstrumented part of the detector, like the beam pipe or a support structure.

This process is easier to simulate than the fake muon class processes, and Monte Carlo techniques are used to determine its contribution. According to [56] there are no processes other than $\mathrm{W}+\gamma^{*} / \mathrm{Z}$ that have a significant contribution in the three muon final state event class. The tree muons give a very clean signal with an expected signal to background ratio of 3.7. Figure 3.7 shows the invariant mass of the $W Z$ system as measured by the detector, including the contributions to the distribution by the background processes. Also, the backgrounds for the

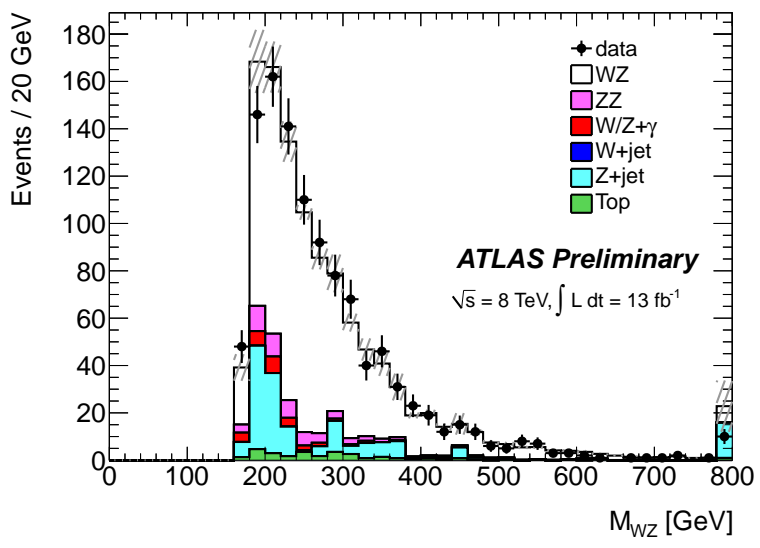

Figure 3.7: The invariant mass of the WZ system as measured by ATLAS [56]. The data points are measured by the detector, the white histogram is the contribution from the $W Z$ events and the colored histograms are the background processes. The difference in size between the white histogram and the colored histograms combined indicates that the signal is very clean.

$W Z$ signal are the same as the backgrounds in this study. Adding the fact that the event selection used in that study is very similar to the ones presented here this process is the main focus in the study of this background class. The Monte 
Carlo event generator used in this study is the same as the $W Z$ study used for the $\mathrm{W}+\gamma^{*} / \mathrm{Z}$ process, which is Sherpa [57].

\subsubsection{Four muon final state: $\mathrm{ZZ}$}

The four muon process class is not as significant as the $\mathrm{W}+$ jets or $\mathrm{W}+\gamma^{*} / \mathrm{Z}$ processes, but still accounts for about $5 \%$ of background events. The single process responsible for this class is the tree-level $\mathrm{Z}$ pair production process. From these events two muons with equal sign escape detection, and two equal sign muons are reconstructed by the detector.

This process has a much smaller contribution to the overall background than the $W Z$ process for two main reasons. First the production of the second $Z$ boson has a much smaller cross section than the $W$ boson, and the probability of two same sign muons escaping detection is also smaller compared to only one muon leaving unseen in the three muon background class.

\subsection{Dataset preparation}

The complete data set recorded by ATLAS during 2012 is used in this analysis. Since most of these events are not interesting for this study, a number of baseline cuts is applied that selects only the events useful in this study. The first requirement in the event selection is that there should be exactly two muons recorded, and they should have the same charge. The full muon reconstruction is identical to the object selection of the Higgs decaying to two W bosons study [58], subsequent cuts applied on the reconstruction variables is given in section 3.4.1. Also there should be no more than 1 jet above $25 \mathrm{GeV}$ detected by the calorimeter system, and the missing transverse momentum of the muon should not be lower than $10 \mathrm{GeV}$. The jet energy threshold is chosen such that the resulting data set can be compared to other studies, and is determined by the calorimeter group to optimally vetoing jets from the underlying event. The $E_{T}^{m i s s}$ cut is chosen to be as relaxed as possible, while still cutting unwanted background like dijet events.

The different event classes have different distributions for the significant variables. It is these differences in distribution that is used to determine the relative contribution of the classes to the total data set. The shape of the distributions is determined beforehand using the event samples described in sections 3.1, 3.3.1 and 3.3.2. These individual samples are then fitted to the distribution of the data from the detector, see section 3.5.2, using the Beeston Barlow [59] method. The basic concept of this algorithm is to use a standard likelihood fit using Poisson statistics, while varying the Monte Carlo templates within their respective uncertainties. This adds for every bin in all templates an extra fit parameter, which are analytically minimized. The result of this fit gives the relative contributions 
of the event classes. In the next sections the fits are shown for the different variables.

\subsubsection{Cuts on muon momentum}

Two muons are required in the event, and it must be plausible that they are produced in $\mathrm{W}$ decay. This means that they should have sufficient momentum, and that they are isolated from other activity in the calorimeter. The muon with the highest momentum, called the leading muon, has to have a transverse momentum of $25 \mathrm{GeV}$ or higher, the lower momentum muon has a transverse momentum requirement of $10 \mathrm{GeV}$. There is also a cut on the rapidity of both muons, which is set on $\eta<2.5$. This is due to the inefficiency of the detector to measure the muons going close to the beam pipe. This cut is the main reason of the background classes with $3+$ muons in the final state. The results of the background contribution fits will be discussed in section 4.1, in this section the histograms are already shown with the background contributions as found in this study. In figure 3.8 the muon transverse momentum is shown, with the leading muon in the left plot, and the subleading muon on the right.
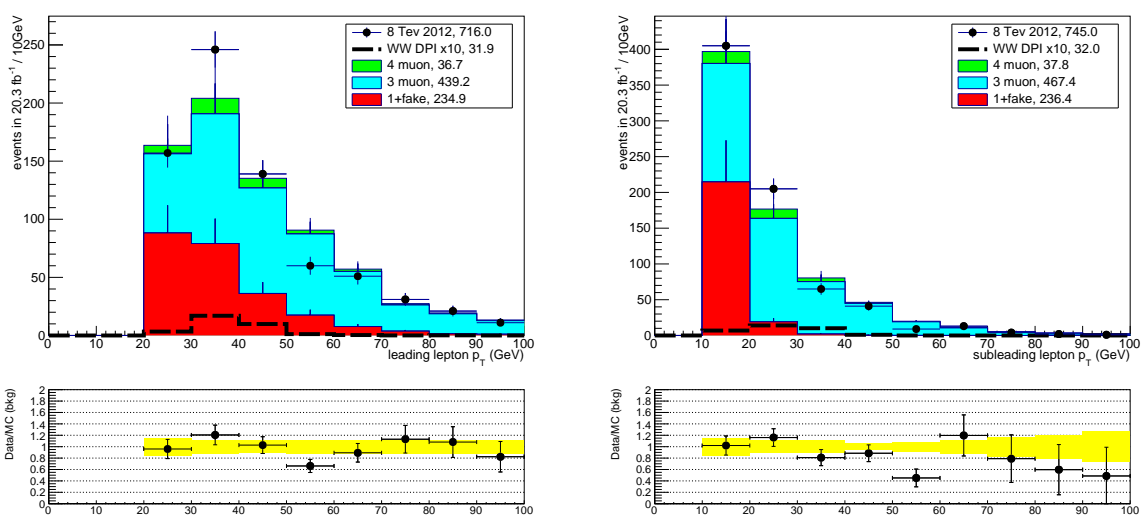

Figure 3.8: Muon transverse momentum with the detector data divided in the different contributions of the background processes. The DPI signal process contribution is multiplied by 10 to enhance visibility. The bottom panel shows the similarity between the simulation and detector data. The data points are all within the statistical error (error bars) and fitting uncertainty (yellow band), except the muon counts with transverse momentum $50<p_{T}<60 \mathrm{GeV}$. 


\subsubsection{Cuts on missing transverse momentum}

The energy of both neutrinos has to be calculated from the missing transverse momentum, as explained in section 3.2. Since the two independent muons can be back-to-back, the total missing transverse momentum from the two neutrinos can be very small. Therefore only a very loose cut on transverse momentum of $10 \mathrm{GeV}$ is put on the initial event selection. Figure 3.9 shows a slight underestimation of the number of events with low $E_{T}^{\text {miss }}$, and an overestimation in the high $E_{T}^{\text {miss }}$ region. This is likely from background processes with low $E_{T}^{\text {miss }}$ that were not considered in this study, like dijet events. In the subplot on the bottom it is shown that the deviations are within fitting uncertainty (yellow band) and statistical errors (error bars), so this will have no significant impact on the results of this study. The $E_{T}^{m i s s}$ is taken quite loose in order to not further restrict statistics.
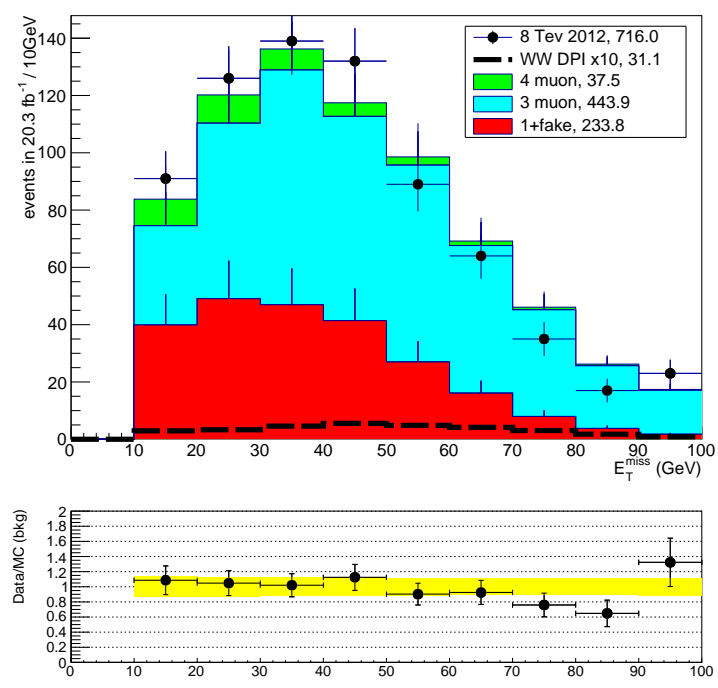

Figure 3.9: Missing transverse momentum. The subplot on the bottom indicates that the background data sets underestimate the number of events with low $E_{T}^{\text {miss }}$, and overestimate the high $E_{T}^{\text {miss }}$ region. However the data points are within the yellow band, which is the fitting uncertainty on the contribution of the two main classes. 


\subsubsection{Cuts on $\mathrm{W}$ boson reconstruction variables}

From the two muon momenta and the missing energy an attempt is made to reconstruct two $\mathrm{W}$ production processes. In case there actually are two $\mathrm{W}$ bosons in the event this will produce better results then when the event was produced by one of the background processes. This can be concluded from the observation that the DPI contribution in the $W Q_{T}$ histograms in figure 3.10 seems to cluster around the $\mathrm{W}$ boson rest mass $(80.4 \mathrm{GeV})$. This makes this variable also interesting to place tight event selection criteria on, specifically the high energy tail can be cut out of this distribution because there are only background events there. However, this study requires a large number of simulated background events. This is further explained in section 4.2. Since computing time is limited, this cut will not be applied on the 2012 data set to retain the necessary statistics of the background samples.
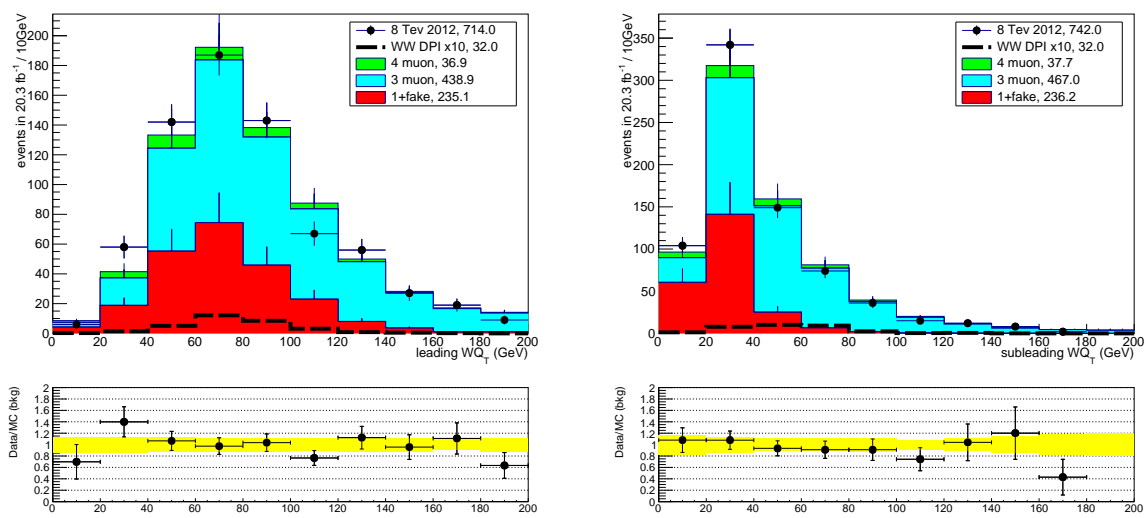

Figure 3.10: $\mathrm{W}$ reconstruction quality $W Q_{T}=\sqrt{4 p_{T}^{\mu} p_{T}^{\nu}}$. The left histogram gives the leading $W Q_{T}$ and the right figure the subleading $W Q_{T}$. Comparison with simulations is very good as can be seen in the lower panel of the graphs. The error bars in this panel are the statistical error, the yellow band is the fitting uncertainty. The DPI signal contribution is clustering around the W mass of 80.4 GEV as expected.

\subsubsection{Additional cuts on data quality}

The full set of cuts is given in tables $3.4,3.5$ and 3.6. The muon calorimeter isolation requirement is a combination of $z_{0} \sin \theta, d_{0}$ and EtConeCor $30 / p_{T}$ and 


\begin{tabular}{|r||l|l|l|l|l|}
\hline variable: & trigger & $p_{T}$ & $\eta$ & $z_{0} \sin \theta$ & $d_{0} \operatorname{sig}$ \\
\hline value: & $\begin{array}{l}\text { mu24i_tight, } \\
\text { mu36_tight }\end{array}$ & $>25 \mathrm{GeV}$, & $<2.5$ & $<1 \mathrm{~mm}$ & $<3$ \\
& $>10 \mathrm{GeV}$ & & & \\
\hline
\end{tabular}

Table 3.4: All muon track cuts applied to the full 2012 data set. The trigger requirement has an $\mathrm{OR}$ relation, the $p_{T}$ cut is given for the leading and subleading muon.

PtCone $40 / p_{T}$ cuts. The former two give a measure of the distance of the reconstructed muon track to the primary event vertex, while the latter two give an indication of the activity in the calorimeter close to the track. The EtConeCor30 variable is the transverse energy sum of all the particles within $R=0.3$ from the muon track, and the PtCone40 is the transverse momentum sum of the particles in a cone with $R=0.4$ around the muon track. These variables are scaled with the muon $p_{T}$ to accommodate the effect that high energy muons generate activity of their own in the calorimeter.

\begin{tabular}{|l|l|l|}
\hline$E_{T}$ & EtCone & PtCone \\
\hline \hline $10-15$ & EtConeCor30 $/ p_{T}<0.06$ & PtCone $40 / p_{T}<0.06$ \\
$15-20$ & EtConeCor30 $/ p_{T}<0.12$ & PtCone $40 / p_{T}<0.08$ \\
$20-25$ & EtConeCor30 $/ p_{T}<0.18$ & PtCone $40 / p_{T}<0.12$ \\
$>25$ & EtConeCor30 $/ p_{T}<0.30$ & PtCone $40 / p_{T}<0.12$ \\
\hline
\end{tabular}

Table 3.5: All muon calorimeter cuts applied to the full 2012 data set.

\begin{tabular}{|r||l|l|l|l|l|}
\hline variable: & jet author & $E_{T}^{\text {jet }}$ & $\eta^{\text {jet }}$ & JVF & $E_{T}^{\text {miss }}$ \\
\hline value: & $\begin{array}{l}\text { anti- } k_{T}, \\
R=0.4\end{array}$ & $>25 \mathrm{GeV}$ & $<4.5$ & 0.5 & $>10 \mathrm{GeV}$ \\
\hline
\end{tabular}

Table 3.6: All jet and $E_{T}^{\text {miss }}$ cuts applied to the full 2012 data set. The JVF variable is the jet vertex fraction, and is the fraction of the momentum contributed by tracks that point back to the primary vertex.

\subsection{Data analysis method}

The analysis consists of three stages graphically shown in figure 3.11. The input data is shown in the leftmost gray column, which consists of the outputs of the event simulation programs including detector simulation, and the data set with 
real detector data. The simulations are run on large computer clusters using the specific ATLAS 2012 parameter tunes.

The middle gray column contains the derived data which is the output from the first analysis steps. These are the ratio of contribution between the two largest background sources, $\mathrm{W}+\gamma^{*} / \mathrm{Z}$ and $\mathrm{W}+$ jets, the Neural Net response templates and the pseudosets. The Neural Net response templates are explained in section 3.5.1, and a pseudoset is a data set of size $n_{d}$ with simulated events where $n_{d}$ is the number of events in the detector data set. The process composition of a pseudoset is according to the $\mathrm{W}+\gamma^{*} / \mathrm{Z} / \mathrm{W}+$ jets ratio, with the number of signal events $n_{D P I}$ varying per pseudoset between zero (null hypothesis) and 200.

The rightmost gray column holds the final analysis output, which is the contribution per background class (1+fake muon, 2 muon, 3 muon) and the signal process.

In between the three gray columns are the distinct analysis steps. In the first step two machine learning classifiers are trained to distinguish events between the two main backgrounds and the signal topology. These classifier responses are used to create a $2 \mathrm{D}$ template of responses to the different event classes. Also the ratio between the $\mathrm{W}+\gamma^{*} / \mathrm{Z}$ and $\mathrm{W}+$ jets processes in the detector data set is determined using the fitting procedure described in section 3.5.2. Using this ratio a large set of pseudo data sets is constructed. The total number of events $n_{d}$ and this ratio is kept constant in these simulated pseudosets, the number of simulated signal events $n_{D P I}$ is varied between zero and 200 .

Finally the 2D templates are fitted to all the pseudosets, and the real data set. The results of the fit on the real data set is compared to the results obtained with the individual pseudosets. Since the pseudosets have an increasing number of signal events starting at zero, the pseudoset that gives the most similar results to the real data set gives an indication to the signal contribution in the latter. The analysis described here is applied on the ATLAS $20128 \mathrm{TeV}$ data set in chapter 4 .

\subsubsection{Signal/background separation with Neural Networks}

The two dominant background classes, the fake muon class and the three muon final state class, account for the vast majority of background events. Therefore a method is developed to distinguish between a signal event and a background event from either of these two background classes. The result of the procedure will be an estimate of the contribution to the data set of the signal process (W pair DPI), the fake muon class $(\mathrm{W}+$ jets) and the three muon final state class $\left(\mathrm{W}+\gamma^{*} / \mathrm{Z}\right)$.

A Neural Network is very suitable for this task of distinguishing between two event types. They can be used to infer a function from observations, giving a 


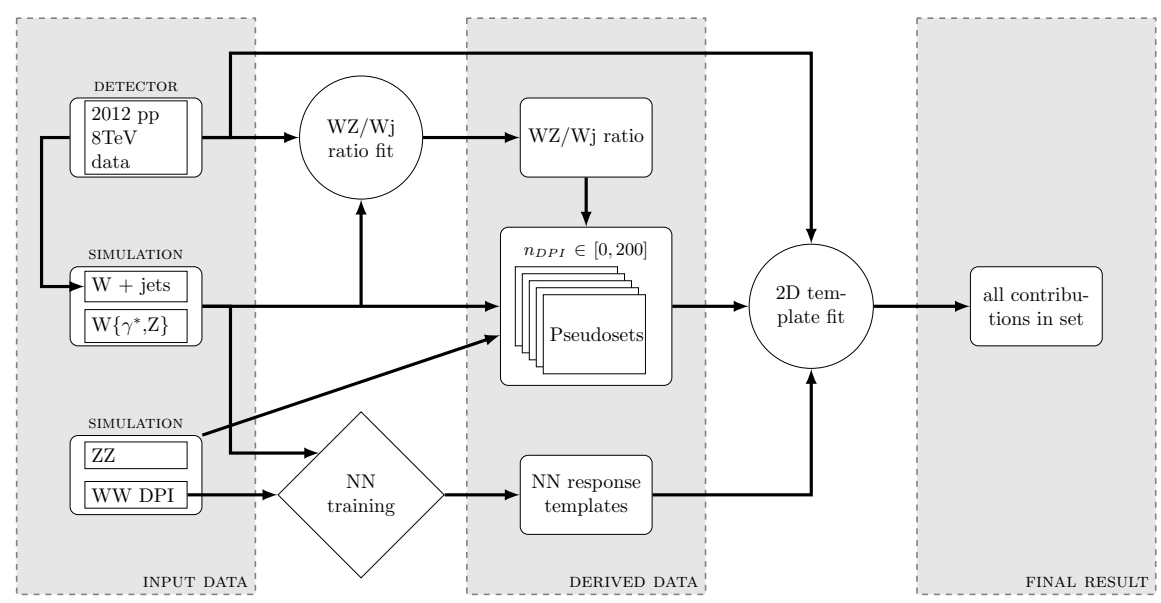

Figure 3.11: Graphical representation of the analysis procedure. The three gray columns indicate data domains in the graph, the diamond and two circles point to analysis procedures. The graph starts on the left with the simulated input data sets and the detector data sets. In the first analysis step the main background ratio is determined and the Neural Net responses and pseudosets are created. From the middle gray column the pseudosets and NN responses are used to determine the final analysis output: the relative process contributions to the data set.The analysis described in this diagram is applied on the ATLAS 20128 $\mathrm{TeV}$ data set in chapter 4 .

single value as output. When the observations are chosen to be the event variables like particle energy, outgoing angle and relative angles between the particles, combinations of these variables are singled out that have high probability of coming from only one type of event class. A Neural Network is trained on a simulated data set with known process composition. The events in the training set are flagged either to be from the background class or from the signal class. The training procedure uses the event variables to optimize the network to assign a low output value to events in the background class, and a high output value to events from the signal class. Finally a cut value $x_{c}$ on the network output $x_{\text {out }}$ is determined for which events with $x_{\text {out }}<x_{c}$ are considered background events, and events with $x_{c}<x_{\text {out }}$ are considered signal events. The last step is simply counting the number of events in the data set that have a response below the cut and above the cut.

A pair of Neural Network classifiers is trained to discriminate events between either a DPI event, or an event from one of the two main background classes. The networks are trained on four variables; the leading and subleading muon 
transverse momentum from figure 3.8, and the $\mathrm{W}$ reconstruction quality (figure 3.10) for the two $\mathrm{W}$ bosons supposed to be in the event. The classifier responses
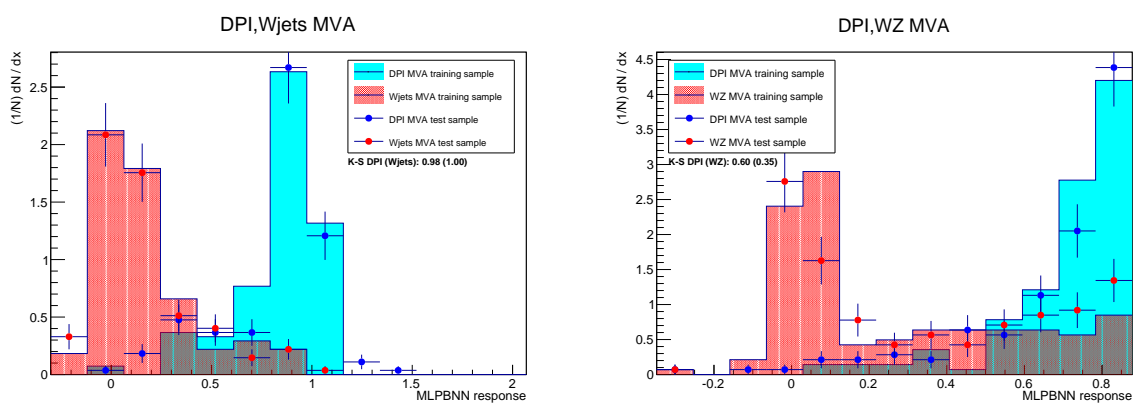

Figure 3.12: Classifier responses of two Neural Networks. The left net is trained to distinguish between $\mathrm{W}+$ jets events and $\mathrm{W}$ pair DPI. The blue background peak is clearly separated from the red signal event peak, with a cut value on the response of around 0.5. The right is trained on $\mathrm{W}+\gamma^{*} / \mathrm{Z}$ and $\mathrm{W}$ pair DPI. Separation is less than for the $\mathrm{W}+$ jets classifier for the intermediate response values, but the signal and background peaks are still distinctly isolated.

are shown in figure 3.12. The left plot shows the training results for the $\mathrm{W}$ + jets background, the right plot contains the responses of the training on the $\mathrm{W}+\gamma^{*} / \mathrm{Z}$ background. The signal response is shown in blue, the background in red. In the left plot it is clear that the Neural Network is very capable of distinguishing between $\mathrm{W}+$ jets events and DPI (signal) events. Almost all W + jet events (red) have a response below 0.5, and almost all simulated DPI events have a response above this value. The classifier has a bit more difficulty with the $\mathrm{W}+\gamma^{*} / \mathrm{Z}$ background, meaning its event topology is very much like the signal event topology. The response on background events clearly peaks at zero in the right plot, and for the signal events around 0.8. In the range between the peaks there is quite a bit of overlap between the signal and background responses giving ambiguous results.

\subsubsection{Data fitting procedures}

Two important steps in the analysis method are the fitting of the two main background classes to the data, and the fitting of the $2 \mathrm{D}$ classifier responses of the background classes to the responses of the detector data. They are indicated by a circle in the diagram in figure 3.11. Both fitting procedures are run using the Beeston Barlow [59] algorithm.

The fit of the ratio between two main background classes $(1+$ fake muon and 
3 muon final states) determines the composition of the pseudosets. It is a one dimensional fit on the distribution of each important variable of the data set, like muon $p_{T}$ (figure 3.8), $E_{T}^{\text {miss }}$ (figure 3.9) and others. The shape of the distributions is kept fixed, the normalization of the two background classes is varied to find a best fit with the detector data. The Beeston Barlow algorithm takes into account the errors given for each bin, introducing an additional degree of freedom for every bin in the fit. These are at a later stage minimized analytically by the algorithm. Each event variable gives a separate result, and the average of results from all variables is the final result of this first fitting procedure. The results of this first fit described above in the ATLAS $20128 \mathrm{TeV}$ data set are given in section 4.1.

The second fit is a $2 \mathrm{D}$ fit of the Neural Network responses. The fit is two dimensional because there are two classifiers used in this analysis. The response of both these classifiers is used to create the $2 \mathrm{D}$ template of both dominant background events and signal events given in figure 3.13. The data sets to create
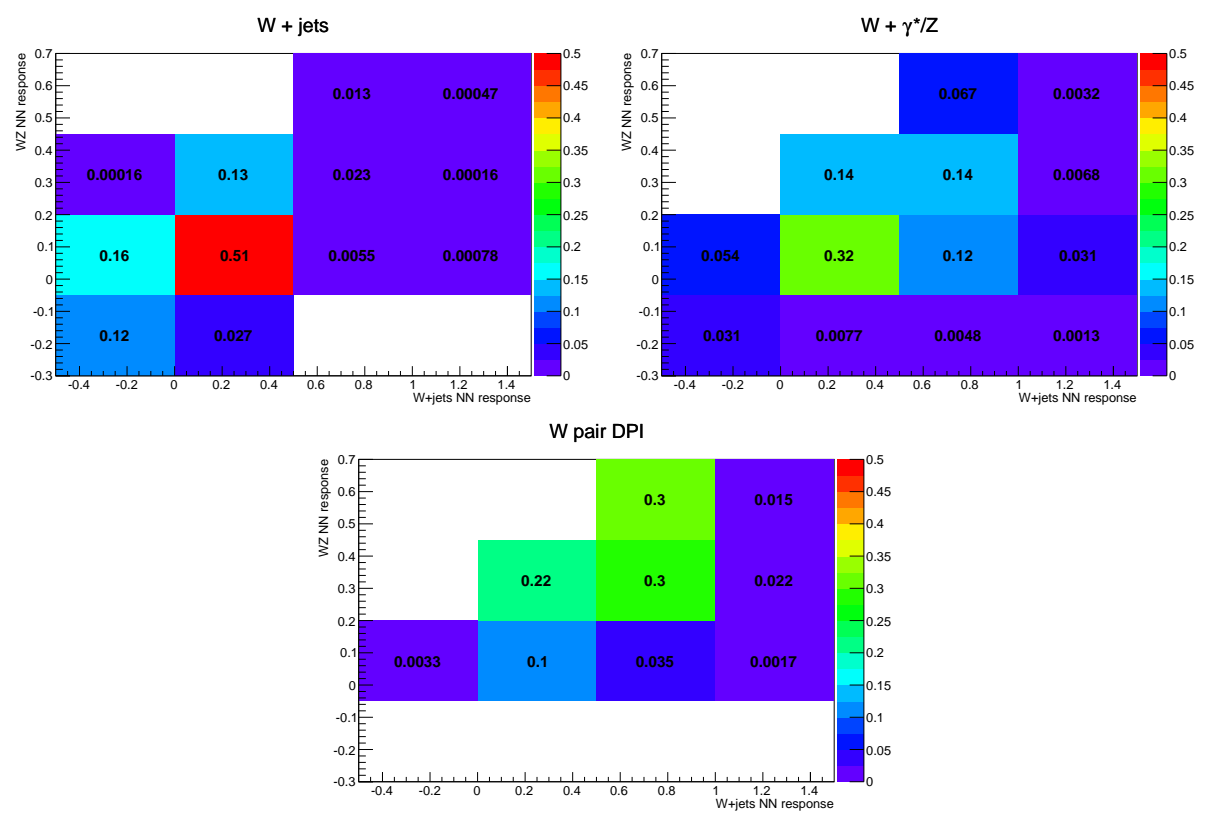

Figure 3.13: Classifier response templates of the DPI signal and the two main background classes. The left plot is the $\mathrm{W}+$ jets template, the right plot is from $\mathrm{W}+\gamma^{*} / \mathrm{Z}$ and the lower middle figure is the signal template from $\mathrm{W}$ pair DPI.

the templates are obtained using the prescription of sections 3.1, 3.3.1 and 3.3.2. The last step to acquire the relative contributions of the three event types is 
fitting the three $2 \mathrm{D}$ templates to the data set. This second fitting procedure is applied to all the pseudosets and the detector data set, to determine which pseudoset is most similar to the ATLAS $20128 \mathrm{TeV}$ data set. These results are given in section 4.2 .

\subsubsection{Summary}

To summarize the procedure we consider the diagram in figure 3.11. We start in leftmost gray column where the input data sets are given. They are obtained using the prescriptions discussed in sections $3.1,3.3$ and 3.4 and generated on large computation clusters using the ATLAS 2012 specific simulation tunes.

The derived data, i.e. the main background ratio, pseudosets and Neural Network responses are obtained from this input data using the $\mathrm{WZ} / \mathrm{Wj}$ ratio fit and the NN training between the left and middle gray column. These results are given in section 4.1 .

To finally arrive at the final result in the right gray column (the different process contributions in the data set) the $2 \mathrm{D}$ template fit of the Neural Network responses is applied on the derived data in the middle gray column. The results are presented in 4.2 and discussed in section 4.3 and chapter 5 . 


\section{4}

\section{ANALYSIS OF W BOSON PAIR PRODUCTION IN DPI EVENTS}

The 2012 ATLAS data set is the largest collection of proton interaction events to date. It has over $20 \mathrm{fb}^{-1}$ collision data at an unprecedented center of mass energy of $8 \mathrm{TeV}$. This makes it the best candidate data set to search for $\mathrm{W}$ pair production from double parton interactions, which are theorized to have a production cross section of a few femtobarn.

In the previous chapter the baseline cuts are explained that are used to filter the events that are for sure not signal. This brings the multi-billion event set of 2012 down to only 750 events. This filtered (signal)set still contains almost exclusively background events. The simulations in this study predict the $\mathrm{W}$ pair DPI signal contribution in this data set to be a couple dozen in the most optimistic case.

These background events are studied using simulations as described in the previous chapter. However, the magnitude of the contribution to the signal data set is difficult to simulate and can only be approximated. This is mainly due to the asymptotic freedom of QCD, which can increase the production rate considerably by introducing alternative production channels with significant probability. The exact magnitude of the individual contributions from the various processes in the data set has to be determined by fitting the distributions of key variables (like muon $p_{T}$ ) to the corresponding distribution obtained from the signal data set.

In section 4.1 this background study and its results, the individual background contributions in the data set, is given. In section 4.2 the null hypothesis and the signal hypotheses are tested. These test give the probability of the data set having zero signal events (null), and the probability of the data set having any number 
between 0 and 200 signal events. The hypothesis with the largest probability of being correct is used to derive a cross section for $\mathrm{W}$ pair production in DPI events, using the simulations described in the previous sections. This cross section estimate is given in section 4.3 .

\subsection{Contamination of the signal data set}

In the previous chapter three background classes that contribute to the signal data set are explained. Two of them, the three muon and four muon final state background classes, are simulated using a Monte Carlo program, which gives an estimate of the magnitude of the respective contributions. The third, the fake muon background class, is assumed to be responsible for the remainder of the events. The four muon final state background class, which is mainly $Z Z$ production events, has a small cross section relative to the three muon final state, the $W+\gamma^{*} / Z$ production events. Therefore the four muon final state contribution is taken according to the $Z Z$ Monte Carlo simulation. Normalizing to $20 \mathrm{fb}^{-1}$, 38 events pass the cuts and enter the signal set of 750 events. This means that in our case of a very small signal contribution, the fake muon and three muon background classes make up for almost all the background events, i.e. $95 \%$ of the signal dataset.

The ratio of the two main background classes in the data set is determined using the Beeston Barlow [59] algorithm. An important assumption in this process is that these two event classes are responsible for all events in the data set, because the minor background and the signal are comparable to the Poisson error on the data points. Inputs to the fit are the data set with muon isolation requirements reversed and the $\mathrm{W}+\gamma^{*} / \mathrm{Z}$ simulated set, which are fitted to the measured data set with the simulated $Z Z$ data set subtracted.

The results of the fit on two variables are shown in figure 4.1, the outcome of the fit on any of the available variables is summarized in figure 4.2. The summary of the fit to all variables shows that a $33 / 67$ ratio is most probable, with the $\mathrm{W}+$ $\gamma^{*} / \mathrm{Z}$ contribution being the largest. The standard deviation on this ratio is 9.0. Using this measurement as a guide the pseudosets will be filled with $38 \mathrm{ZZ}$ events as given from the MC simulation using a k-factor of 1 , the signal event count will be increased from 0 to 200 and the remainder of the 750 events will be $33 \%$ fake muon events and $67 \%$ events from the three muon background class. This $33 / 67$ ratio is varied randomly between $24 / 76$ and $42 / 58$ with a uniform distribution to take into account the standard deviation on the ratio found. 

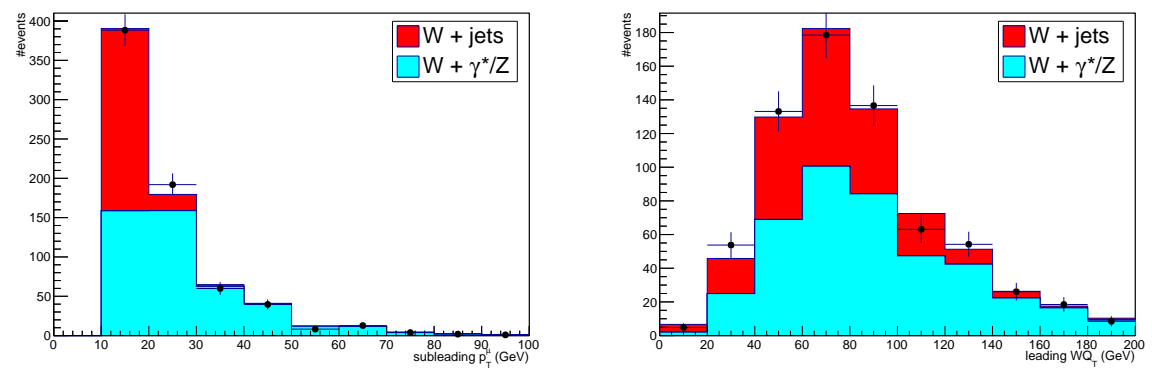

Figure 4.1: Result of the Beeston Barlow fitting algorithm using the subleading muon $p_{T}$ variable on the left, and the leading $\mathrm{W}$ reconstruction quality variable on the right. The black markers indicate the number of events in the detector data set.

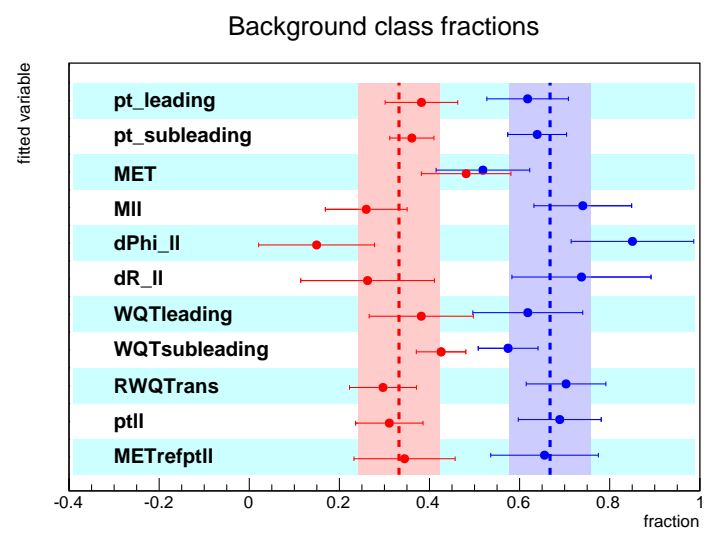

Figure 4.2: Fit results of the ratio between the fake muon class (red) and the three muon class (blue) contributions. It is assumed that the whole data set consists of only these two event classes, since the other backgrounds and the signal are comparable to the statistical error on these two. The ratio of the contributions of these two event classes is found to be $33 / 67$, with a standard deviation of 9.0 . This error is shown as a red and a blue band.

\subsubsection{Fake background class validation}

The previous section gives results that can be used to validate the fake muon background set. The data set with events that passed all the cuts is taken as a basis, from where 38 random $Z Z$ events are subtracted. Dividing the remaining 
events with a ratio of $33 / 67$ over the two main background processes there are 235 events from the fake muon background class and 447 events from the three muon background class in this data set. For the $\mathrm{W}+\gamma^{*} / \mathrm{Z}$ process this gives a $\mathrm{k}$-factor of $1.79 \pm 0.24$.

After subtracting this three muon histogram from the data set as well the only remaining source of events should be from the $1+$ fake muon background class. The resulting variable distributions are consistent with the distributions from the data set with inverted muon isolation requirements, as shown in figure 4.3. To test compatibility between the remaining events distributions and the
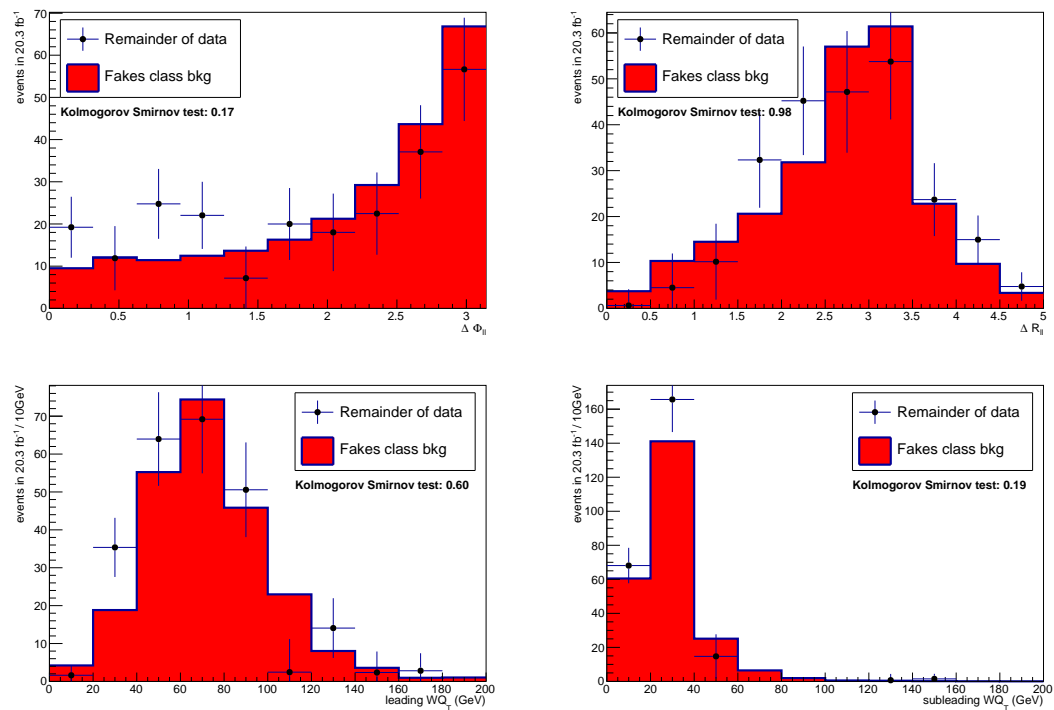

Figure 4.3: Distribution validation of the data driven fake muon class data set generation. The top left histogram is the validation on the $\Delta \phi$ between the muons and the top right figure shows $\Delta R$ between them. This shows the muon directions are correctly modeled. The bottom two figures show the $\mathrm{W}$ reconstruction quality, which indicate that the event topology is also correct.

data set with inverted muon isolation the Kolmogorov Smirnov (KS) [60] test is used. The result of this test is a uniformly distributed value between zero and one for compatible histograms, i.e. the probability of the test. A value much less than zero means not compatible.

A summary of the KS factors of the other distributions is given in table 4.1. The table shows six tests result with high probability of compatibility, and five with a lower probability. The variables connected to the event topology $(\eta, \phi)$ 
have better results than the variables of energy magnitude $\left(p_{T}, E_{T}\right)$, and the variables that are a combination of these $\left(W Q_{T}\right)$ have an intermediate goodness of fit. The topology variables give confidence that the data set with inverted muon isolation is a good candidate for representing the $1+$ fake muon background, except for the absolute energies of the particles. Since the muons in this data set are required to have a lot of other activity in close proximity, it is assumed that the average energy of these muons is lower than the muons in the isolated muon data set. In a follow-up study it is recommended to determine a scale factor for the muon momenta to improve the compatibility in these variables as well.

\begin{tabular}{|l|c|}
\hline variable & KS value \\
\hline$p_{T}^{\text {leading }}$ & 0.10 \\
\hline$p_{T}^{\text {subleading }}$ & 0.14 \\
\hline$E_{T}^{\text {miss }}$ & 0.22 \\
\hline$M_{\ell \ell}$ & 0.79 \\
\hline$\Delta \phi_{\ell \ell}$ & 0.17 \\
\hline$\Delta R_{\ell \ell}$ & 0.98 \\
\hline$W Q_{T}^{\text {leading }}$ & 0.60 \\
\hline$W Q_{T}^{\text {subleading }}$ & 0.19 \\
\hline$W Q_{T}$ ratio & 0.99 \\
\hline$p_{T}^{\ell \ell}$ & 0.99 \\
\hline$E_{T}^{\text {miss }}$ ref $p_{T}^{\ell \ell}$ & 1.00 \\
\hline
\end{tabular}

Table 4.1: KS values of the distributions used to validate the data driven fake muon class data set generation. The values are the probability of the give test, a number much lower than one means not compatible. The $M_{\ell \ell}$ variable is the reconstructed mass of the two lepton system, the other variables are previously explained throughout the text. The variables connected to the event topology $(\eta$, $\phi)$ have better results than the variables of energy magnitude $\left(p_{T}, E_{T}\right)$, and the variables that are a combination of these $\left(W Q_{T}\right)$ have an intermediate goodness of fit. The topology variables give confidence that the dataset with inverted muon isolation is a good candidate for representing the $1+$ fake muon background, except for the absolute energies of the particles. Since the muons in this data set are required to have a lot of other activity in close proximity, it is assumed that the average energy of these muons is lower than the muons in the isolated muon data set. 


\subsection{Null hypothesis and signal hypotheses}

In case a signal is expected to be very difficult to extract, it is sometimes better to study if the null hypothesis, or absence of signal, can be rejected. This is the approach taken here. In order to refute the null hypothesis an ensemble of pseudo data sets with only events from the background classes is constructed. These data sets have the relative contributions of the classes as determined earlier, and the same number of events as the signal data set obtained from the detector. Every pseudo data set is fed to the two neural networks detailed in section 3.5.1, where a $2 \mathrm{D}$ distribution of both network responses is constructed. The average of the distributions for the null hypothesis is shown in figure 4.4. This $2 \mathrm{D}$ distribution

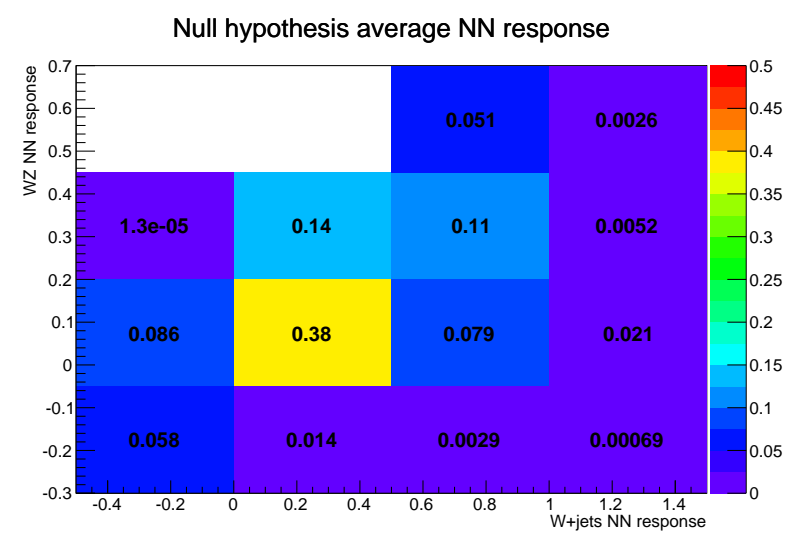

Figure 4.4: Average classifier response template to the pseudosets without signal contribution (null hypothesis).

of responses is then fitted to the responses of the fake muon and three muon background classes and the responses of signal events, as shown in figure 3.13. From this fit the relative contributions of these three types of events is calculated. Since there is an ensemble of pseudosets, the error on these fractions can be determined from the variance that is found by repeating the analysis on all the sets in the collection. The fractions found in the pseudosets are shown in the histograms in figure 4.5, and the mean values and the standard deviation is summarized in table 4.2. It turns out that the analysis described above finds a large signal contribution even in the absence of signal events. This is due to the fact that the neural networks trained in this study have, especially in the three muon final state class, a large number of false positives. In these cases the neural network classifies a $W+\gamma^{*} / Z$ production event as a DPI event.

The DPI fractions in table 4.2 indicate that the value for the DPI fraction 

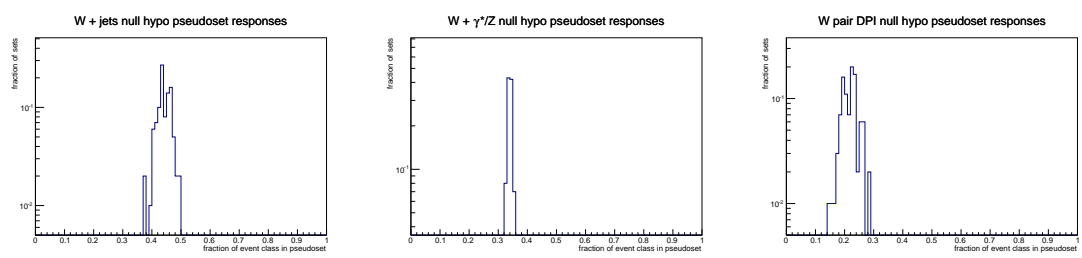

Figure 4.5: Event class fractions found by the Neural Nets of the pseudosets without signal, i.e. the null hypothesis. The distribution of fractions is larger for the signal event class and the fake muon background class as it is for the three muon background class.

\begin{tabular}{|l|l|l|l|}
\hline fractions: & DPI & fake muon bkg & 3 muon bkg \\
\hline 2012 data & 0.231 & 0.425 & 0.345 \\
null hypothesis & $0.219 \pm 0.027$ & $0.442 \pm 0.024$ & $0.340 \pm 0.007$ \\
\hline
\end{tabular}

Table 4.2: Null hypothesis template fit results.

obtained with the ATLAS data set is well within the $95 \%$ confidence interval (green band) of the null hypothesis. Therefore the null hypothesis can not be rejected and is considered to be valid. The $W$ pair from DPI contribution to the data set can not be seen in the 8TeV 2012 ATLAS data set using the analysis procedure followed in this study.

When a null hypothesis can not be rejected an upper limit can be set, in this case on the production cross section. To do this, pseudosets with an ever increasing number of signal events are created in the same way as the pseudosets for the null hypothesis are constructed. The signal events are generated with the Pythia event generator, and a full detector simulation is run subsequent to the generation of the events. The number of signal events added to the pseudosets in the ensembles is increased by five, and the resulting DPI fractions found and the deviations on that fraction is given in figure 4.6. When the analysis is run on the signal data set from the detector, the found fraction can be compared with the results from the pseudosets. This can then be directly translated to a number of signal events in the signal set. The red dashed line in figure 4.6 denotes the fraction found in the signal set from the detector, indicating that the pseudosets with 15 signal events are most similar with the real data. Figure 4.7 shows the 2D Neural Net responses of the this data set on the left, and the average response of the pseudosets with 15 signal events on the right. Table 4.3 gives a summary of the fractions obtained with the 15 signal events hypothesis. 


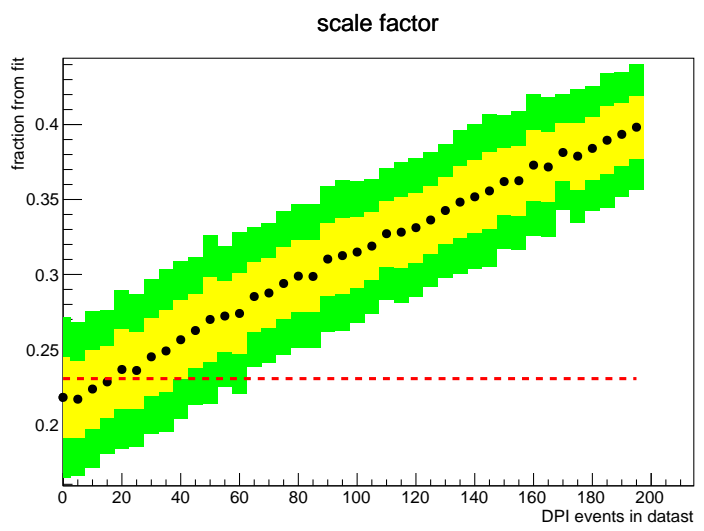

Figure 4.6: Pseudoset ensemble DPI fractions. The yellow band shows the standard deviation in the fraction found for pseudosets with the same number of signal events added, the green band is the 95\% CL. The red dashed line indicates the value found for the detector dataset.
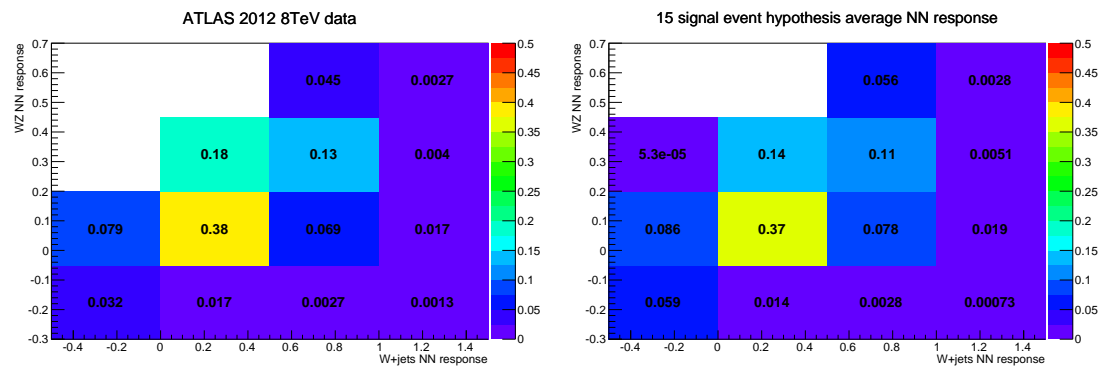

Figure 4.7: Neural Net responses for the detector data set on the left and the average responses of the 15 signal events hypothesis ensemble on the right.

\begin{tabular}{|l|l|l|l|}
\hline fractions: & DPI & fake muon bkg & 3 muon bkg \\
\hline 2012 data & 0.231 & 0.425 & 0.345 \\
15 event hypo & $0.228 \pm 0.024$ & $0.436 \pm 0.020$ & $0.336 \pm 0.007$ \\
\hline
\end{tabular}

Table 4.3: 15 signal events hypothesis template fit results 


\subsection{The DPI W pair production cross section}

The results from the analysis described in section 3.5 show that the null hypothesis can not be rejected. This means that this analysis does not have enough statistics to prove the existence of any signal process, like $\mathrm{W}$ pair production from DPI.

In section 4.2 the 15 event hypothesis is shown to give results closest to the ATLAS data set. However, the pseudoset with largest signal event contribution where a DPI fraction of 0.231 is found within the $95 \%$ confidence interval is the 60 signal event ensemble. This is used in the following text to construct an upper limit to the DPI W pair production cross section.

The signal yield of the analysis is tested with the same Monte Carlo simulated events that were described in section 4.2 to add signal events to the pseudosets. The fraction $R_{\text {yield }}$ is extracted from that using the same cuts as applied in this analysis.

$$
R_{\text {yield }}=\frac{n_{\text {sig }}}{\sigma_{W W}^{D P I}}
$$

In the figures in section 3.4 only 3.2 events from this Pythia Monte Carlo set pass the event selection cuts. The reported production cross section for this data set is $10.9 \mathrm{fb}$, however this Monte Carlo sample includes all $W \rightarrow(\mathrm{e}, \mu) \nu$ final states. The only final state in the analysis is the same sign muon final state, so the reported production cross section is divided by 8 assuming equal production cross sections for $W \rightarrow \mathrm{e} \nu$ and $W \rightarrow \mu \nu$ [55]. This assumption gives an $R_{\text {yield }}=3.2 * 8 / 10.9=2.35$. Using the ensemble with 60 signal events an upper limit on the cross section is found:

$$
\sigma_{W W}^{D P I}<26 \mathrm{fb},(95 \% \mathrm{CL})
$$

The production cross section reported by Pythia for DPI interactions is in general considered to be a factor $2 \mathrm{x}$ too low, and the upper limit found is well above this. If we consider the expected cross section from the factorized DPI cross section for equal $A$ and $B$ particles, using the $W \rightarrow \mu \nu$ cross section from [55] and $\sigma_{\text {eff }}$ as determined in section 1.2 :

$$
\sigma_{D P I}=\frac{1}{2} \frac{\sigma_{A} \sigma_{B}}{\sigma_{e f f}} \approx \frac{(10 \mathrm{nb})^{2}}{2 \times 19 \mathrm{mb}}=2.6 \mathrm{fb}
$$

This value is higher than the cross section reported by Pythia $(10.9 / 8=1.36 \mathrm{fb})$, but still well under the upper limit found in this analysis.

With the null hypothesis still valid, an attempt is made to estimate the amount of data needed to reject it assuming that the most probable signal yield is actually correct. New pseudosets are created, with event counts 4 times and 
10 times the size of the current ATLAS data set. The analysis was performed on these ensembles, keeping the fraction of signal as found in the detector data set. Figure 4.8 shows the pseudoset DPI fractions found in these ensembles.
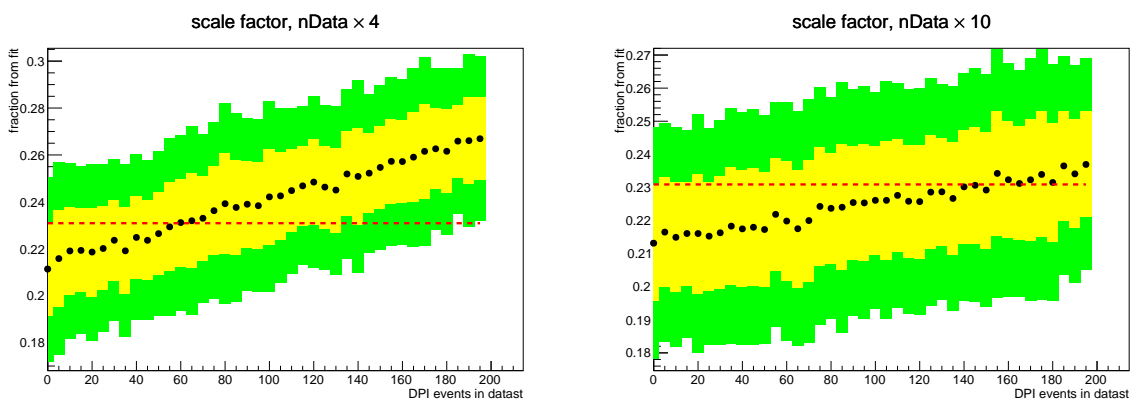

Figure 4.8: Pseudoset ensemble DPI fractions using 4x (left) and 10x (right) the amount of data currently recorded. The red dashed line is the fraction found with the current data.

Comparing figure 4.8 with 4.6 shows that the most probable signal contribution scales by 4 and 10 respectively, like the size of the ensembles. It is also clear that just recording more data will not be enough. It is equally important to reduce the background contributions in the data set. Figure 4.9 shows the prime candidate to apply an extra cut. The ratio of the $\mathrm{W}$ reconstruction quality of both processes gives a good separation between the signal process and the fakes background class. All DPI events are below $r=2$, placing a cut there will reduce the fake background class contribution considerably. The $\mathrm{W}+\gamma^{*} / \mathrm{Z}$ background will then be the sole main contribution. The Neural Network classifiers however do not distinguish between signal and background as efficient as for the fakes background, so a different approach has to be taken when more data is recorded.

Studies investigating quark correlations, see for example [12], are conducted that analyze the effect of partonic correlations in the $\mathrm{W}$ pair production process. Unfortunately there are a lot of unknown variables in the double parton distribution functions that will influence the event yield. However there are observables, like the ratio of events where the $\mathrm{W}$ pair decay products traverse the same or opposite hemisphere of the detector, that can provide clues as to whether these correlations are important or negligible. Further study of these correlations is necessary to determine the validity of the confidence limit found in this analysis. 

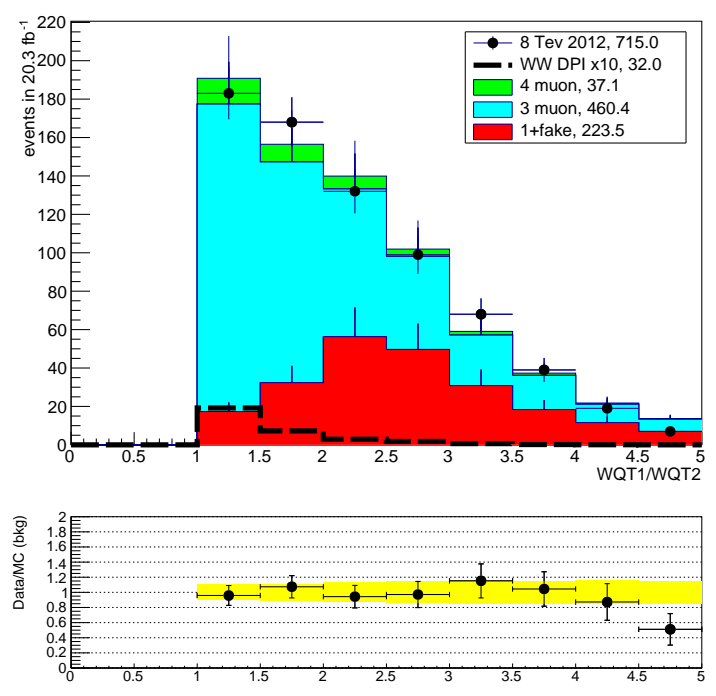

Figure 4.9: The ratio of the $\mathrm{W}$ reconstruction quality of both processes. All DPI events are below 2 , placing a cut at $<2$ will reduce the fake background class contribution considerably.

\subsection{Conclusion}

The method described in this study is a very sensitive tool to probe $\mathrm{W}$ pair production from DPI events. The assumption of using only two background event classes simplifies the error treatment of the $\mathrm{W}+$ jets background. The statistical errors are conveniently recovered using the pseudoset ensemble method, which proves to give a stable indication of the number of signal events in the data set.

The final result of the analysis proves that the current ATLAS 2012 data set of $20.3 \mathrm{fb}^{-1}$ is not sufficient to demonstrate the existence of same sign $\mathrm{W}$ pair production in DPI events. The result of the ATLAS data set is within the $95 \%$ confidence interval of the null hypothesis and has to be considered a background fluctuation.

The main background processes are detector inefficiencies, specifically the three muon background class with the $\mathrm{W}+\gamma^{*} / \mathrm{Z}$ process as the main contributor, and the fake background class with the $\mathrm{W}+$ jets process being responsible for the rest of the events. With more data a new cut can be introduced to the W boson reconstruction quality that greatly reduces the contribution of the fakes 
background class. The three muon background class however is more difficult to separate from the signal process.

An upper limit to the same sign W pair DPI production cross section with two muon final state is set at:

$$
\sigma_{W W}^{D P I}<26 \mathrm{fb},(95 \% \mathrm{CL}) .
$$




\section{CONCLUSION AND PROSPECTS}

The analysis described in the preceding text is performed on the ATLAS 2012 $20 \mathrm{fb}^{-1} 8 \mathrm{TeV}$ proton-proton data set, the largest of such event samples to date as of this writing. It is designed to first establish proof that Double Parton Interactions produce same sign $\mathrm{W}$ boson pairs, and second to extract information about the proton structure from these events.

The signal events are chosen as equal charge prompt muon pairs, which are very unique from a physics point of view. The only known Standard Model process that can create such an event is the $\mathrm{W}$ boson pair production in DPI. However, detection difficulties cause contamination of the data set. This contamination comes from events with different muon multiplicities, which are much more abundant.

Supplementary to the uniqueness of this final state the muon pair is selected for its probing potential of the proton structure. The outgoing angle of the muons produced by the $\mathrm{W}$ boson decay is thought to depend on the spin correlations of the quarks inside the colliding protons, which becomes measurable in double parton interactions. Theoretical work described in the first chapters indicates that the effect can be as large as $10 \%$ in the muon decay hemisphere ratio.

\subsection{Interpretation of results}

To provide evidence of $\mathrm{W}$ pair production in DPI the null hypothesis is tested. This hypothesis claims that the data set acquired from the detector does not contain signal events, i.e. DPI events producing two same sign $\mathrm{W}$ bosons which decay to muons. The events in this null set are divided into three categories, 
based on the multiplicity of prompt muons in the process. The first background class has one prompt muon, and one muon from a different source. The $W+j e t s$ process is responsible for the bulk of events in this class. The second class consists of events with three prompt muons in the final state, where one of them is not seen by the detector. This event class is almost exclusively formed by the $W+\gamma^{*} / Z$ process. These two classes are responsible for up to $95 \%$ of all background events in this analysis. A third background class, with four prompt muons from $Z Z$ production, is taken into account but has limited effect on the results.

The interpretation of figure 5.1 is that the ratio of the relative contributions of the first and second background classes is about 1:2. The ratio is obtained by fitting all the relevant distributions of the two background data sets to the detector data set.

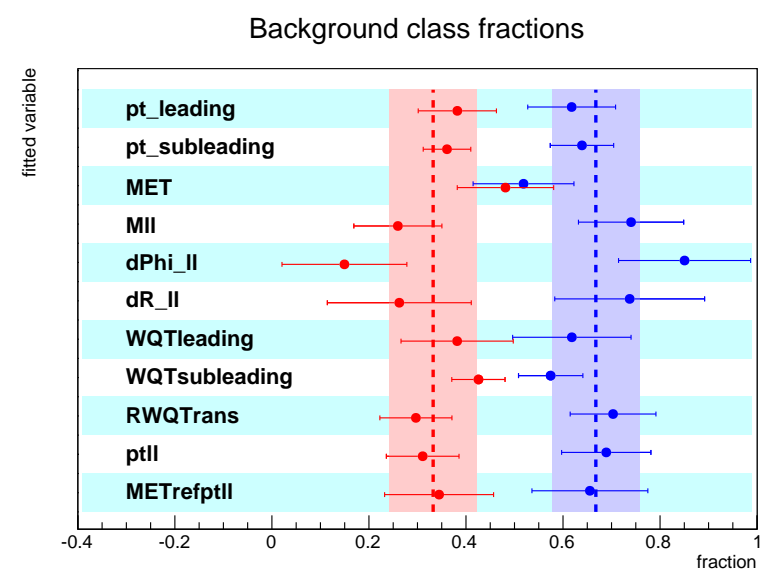

Figure 5.1: Fit results of the ratio between the fake muon class (red) and the three muon class (blue) contributions. It is assumed that the whole data set consists of only these two event classes, since the other backgrounds and the signal are smaller than the statistical error on these two. The ratio of the contributions of these two event classes is found to be $33 / 67$, with a standard deviation of 9.0. This error is shown as a blue and red band.

Using the previously determined contributions of the background classes to the detector data set, the null hypothesis is created. The null hypothesis is a fit result of the classifier responses of all the background classes separately to the detector data set. This fit is designed to give the relative contributions of the different backgrounds. The first bin in figure 5.2 is the result of the null hypothesis test. The other bins in the figure are signal hypothesis tests and the red line is the actual test. With the red line firmly in the $95 \%$ confidence 
limit, the null hypothesis is valid. This means the analysis is not able to confirm the existence of other processes in the data set besides events from the three background classes.

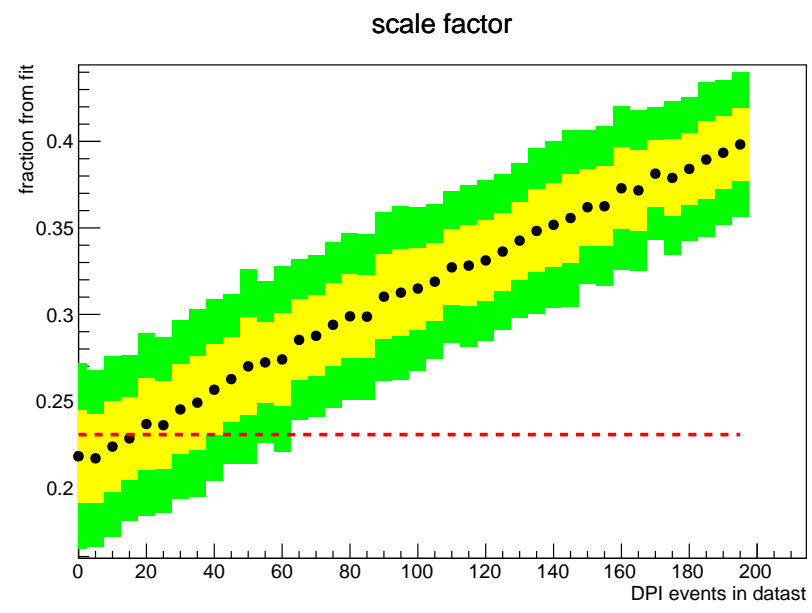

Figure 5.2: Pseudoset ensemble DPI fractions. The yellow band shows the standard deviation in the fraction found for pseudosets with the same number of signal events added, the green band is the $95 \%$ CL. The red dashed line indicates the value found for the detector dataset.

The signal hypotheses results in figure 5.2 gives a most probable signal yield of 15 events in the data set of 750 events in size, and provides an upper limit to the same sign $W \rightarrow \mu$ pair production from DPI events cross section:

$$
\sigma_{2 W \rightarrow \mu}^{D P I}<26 \mathrm{fb},(95 \% \mathrm{CL}) .
$$

The overall result indicates that the DPI events are not easily distinguished from the background processes. The total size of the signal data set is 750 , with an 95\% upper limit of 60 DPI events. This means that the background contribution is still huge after the initial cuts. The Neural Network classifiers are quite able to distinguish between DPI signal and the fake muon background class, but have difficulty telling apart signal and the three muon background class. Reconstructing the two $\mathrm{W}$ bosons suggests that the baseline cuts can be optimized further. Figure 5.3 shows the distributions of the ratio between the leading and subleading $\mathrm{W}$ boson reconstruction result. The signal events are clustered between a ratio of 1 and 2, while the background classes extend well beyond a ratio of 5 . 

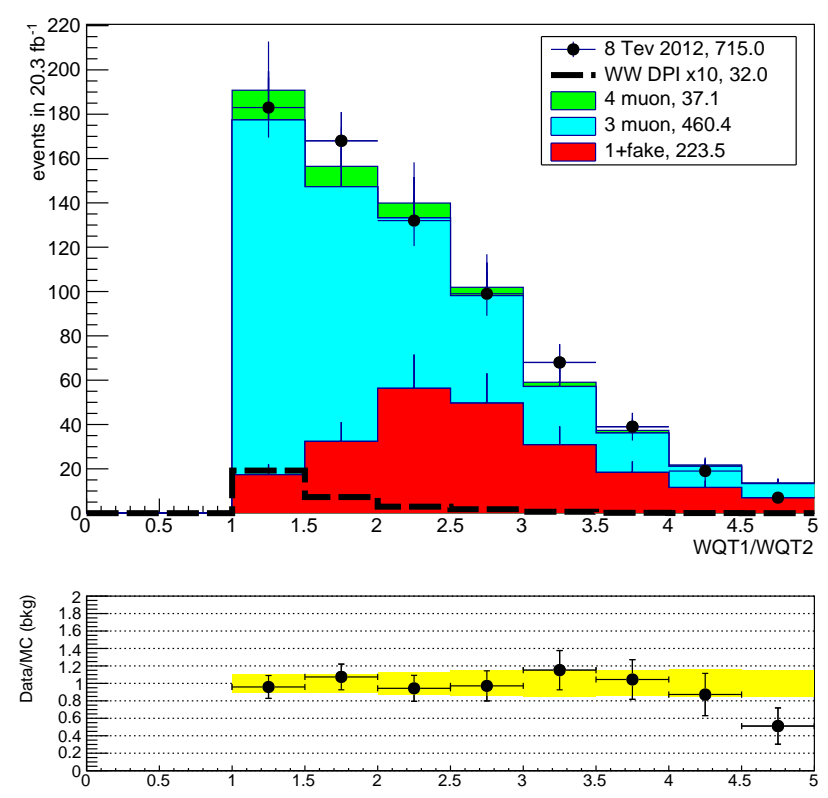

Figure 5.3: The ratio of the $\mathrm{W}$ reconstruction quality of both processes. All DPI events are below 2 , placing a cut at $<2$ will reduce the fake background class contribution considerably.

A proven signal from $\mathrm{W}$ pair production in DPI has the potential to shed light on the quark polarizations inside the proton. Exploiting the exclusively lefthandedness of the weak interaction mediated by the $\mathrm{W}$ bosons the contribution of longitudinal polarization of the quarks can be investigated using the decay angles of the muons.

\subsection{Prospects and recommendations for further research}

The ATLAS detector is scheduled to run for multiple decades more to come. The increase in statistics and center of mass energy will make the DPI contribution in W boson pair production measurable. With increasing center of mass energy the production cross section of DPI events will grow faster than the cross sections 
of the background classes (see [3]), which will increase the signal to background ratio. Improved statistics will allow further research to tighten the event selection cuts, which should also reduce the background contribution significantly.

From the baseline cuts the missing transverse energy cut should be improved first. The performance of the $E_{T}^{\text {miss }}$ below $40 \mathrm{GeV}$ degrades rapidly when going to lower values, which in turn will affect the $\mathrm{W}$ boson reconstruction in the event. This $\mathrm{W}$ boson reconstruction is a very sensitive cut on its own. Figure 5.3 indicates that the ratio of the leading and subleading values for the reconstruction quality in an event has significant predictive power do distinguish DPI events from background events.

The background class with one prompt muon and one fake muon can be improved upon by decomposing it further into individual contributions from different processes. The main contribution is $W+$ jets production, but also the $t \bar{t}$ and $b \bar{b}$ processes are present in this class. In the current analysis this background class accounts to only one third of the total background, and this decomposition would not provide an improvement to the final result. The cut on $\mathrm{W}$ reconstruction quality will further reduce this contribution, however a large increase in statistics in future runs of the LHC may necessitate a better specification of this background class.

The three muon background class is the largest contamination to the signal data set. Unfortunately the Neural Nets have the largest difficulty separating exactly this process from the DPI process. Future effort should start with reducing the impact of this background class. The $W+\gamma^{*} / Z$ process is relatively well understood and gives a clear signal in the detector. It also has proven Monte Carlo generators that provide sound benchmarks.

When statistics improve in the coming data sets from the experiment, the four muon background class will become a significant source of background events as well. This is especially the case when the cuts on the key variables will be set tighter. It will not be possible anymore to describe the full background with only two background classes, so the exact analysis procedure applied currently will not be feasible anymore.

Considering the arguments given above a future analysis of the DPI events in the same sign muon final state should be a more traditional approach, especially if the fake muon class has a small contribution. This class has a very large statistical error compared to the background processes that can be modeled using Monte Carlo simulations, but when the contribution is relatively small, so will be the effect of its systematics.

A further improvement that should be considered when higher statistics allow tighter cuts is the addition of the electron channel. W bosons decay with almost equal probability to electrons and muons but electrons have a much higher chance of having their charge misidentified. Increased statistics will allow tighter cuts to 
reduce this effect to acceptable levels and thereby increasing the signal data set four times including the electron-muon combinations.

If the same sign $\mathrm{W}$ boson from DPI signal can be extracted from future data sets, it will give exciting prospects for probing the proton structure. The DPI process has the potential of giving information about the spin correlation of the constituent quarks no other process can provide at this moment. This will enhance our understanding of QCD and will improve the parton density functions used by virtually every Monte Carlo event simulation program in use today. The ratio of decay leptons traversing the same detector hemisphere and the particles traveling through opposite hemispheres will give key insights into the spin correlations. The effect is estimated to be around $10 \%$. 


\section{BIBLIOGRAPHY}

[1] Combined Measurement of the Higgs Boson Mass in pp Collisions at $\sqrt{s}=7$ and $8 \mathrm{TeV}$ with the ATLAS and CMS Experiments, Aad, G. et al. (ATLAS Collaboration, CMS Collaboration) Phys. Rev. Lett. 114, 191803 (2015)

[2] 2015 Review of Particle Physics, K.A. Olive et al. (Particle Data Group), Chin. Phys. C, 38, 090001 (2014) and 2015 update.

[3] Same-sign $\mathrm{W}$ pair production as a probe of double parton scattering at the LHC, J. Gaunt, C-H. Kom, A. Kulesza, W. Stirling, Eur. Phys. J. C 69, 53-65 (2010)

[4] The measurement of the Higgs self-coupling at the LHC: theoretical status, J. Baglio, A. Djouadi, R. Gröber, M.M. Mühlleitner, J. Quevillon, M. Spira JHEP 1304 (2013) 151

[5] Developments In The Quark Theory Of Hadrons, G. Zweig, vol. 1, 22-101, eds. D.B. Lichtenberg and S.P. Rosen; CERN Geneva - TH. 401 (REC.JAN. 64) $24 \mathrm{p}$

[6] A schematic model of baryons and mesons, M. Gell-Mann, Phys. Lett. 8 (1964) 214

[7] Ultraviolet Behavior of Non-Abelian Gauge Theories, D. J. Gross and F. Wilczek, Phys. Rev. Lett. 30 (1973) 1343.

[8] Reliable Perturbative Results for Strong Interactions? H. D. Politzer, Phys. Rev. Lett. 30 (1973) 1346. 
[9] The Quark and Gluon Structure of the Proton, E. Perez and E. Rizvi, Rep. Prog. Phys. 76 (2013) 046201

[10] D0 Run IIB upgrade technical design report, The D0 Collaboration, (2002) FERMILAB-DESIGN-2002-01

[11] The CDF IIb Detector, Technical design report, The CDF IIb Collaboration, (2002) FERMILAB-TM-2198

[12] Angular correlations in the double Drell-Yan process, T. Kasemets, M. Diehl, JHEP 01 (2013) 121

[13] Trilinear couplings among the electroweak vector bosons and their determination at LEP2, M. Bilenky, J.-L. Kneur, F.M. Renard, D. Schildknecht, Nucl. Phys. B409 (1993) 22

[14] Elements of a theory for multiparton interactions in QCD, M. Diehl, D. Ostermeier, A. Schäfer, JHEP 03 (2012) 089

[15] Double parton scattering in pp collisions at $=63 \mathrm{GeV}$, The AFS collaboration, Z. Phys. C 34 (1987) 163.

[16] A study of multi-jet events at the CERN pp collider and a search for double parton scattering, UA2 Collaboration, Phys. Lett. B 268 (1991) 145

[17] Study of four-jet events and evidence for double parton interactions in $p \bar{p}$ collisions at $\sqrt{s}=1.8 \mathrm{TeV}, \mathrm{F}$. Abe et al, Phys. Rev. D 47 (11) (1993) 4857

[18] Measurement of Double Parton Scattering in $p \bar{p}$ Collisions at $\sqrt{s}=1.8 \mathrm{TeV}$, F. Abe et al, Phys. Rev. Lett. 79(4) (1997) 584

[19] Double parton interactions in $\gamma+3$ jet events in $p \bar{p}$ collisions at $\sqrt{s}=$ 1.96TeV, V. M. Abazov et al (The D0 Collaboration), Phys. Rev. D 81 (2010) 052012

[20] Observation of double charm production involving open charm in pp collisions at $\sqrt{s}=7 \mathrm{TeV}$, R. Aaij et al (LHCb Collaboration), JHEP 1206 (2012) 141, [Addendum: JHEP 1403 (2014) 108].

[21] Study of double parton scattering using $\mathrm{W}+2$-jet events in proton-proton collisions at $\sqrt{s}=7 \mathrm{TeV}$, CMS Collaboration, JHEP 1403 (2014) 032.

[22] A measurement of hard double-partonic interactions in $\mathrm{W} \rightarrow \ell \nu+2$ jet events using the ATLAS detector at the LHC, ATLAS-CONF-2011-160, The ATLAS Collaboration 
[23] Luminosity-Independent Measurement of the Proton-Proton Total Cross Section at $\sqrt{s}=8 \mathrm{TeV}, \mathrm{G}$. Antchev et al (TOTEM Collaboration), Phys. Rev. Lett. 111, 012001

[24] Correlations in double parton distributions: effects of evolution, M. Diehl, T. Kasemets, S. Keane, JHEP 05 (2014) 118

[25] Positivity bounds on double parton distributions, M. Diehl, T. Kasemets, JHEP 05 (2013) 150

[26] ATLAS Collaboration, Expected Performance of the ATLAS Experiment Detector, Trigger and Physics, arXiv:0901.0512

[27] CMS Physics TDR, Volume II: CERN-LHCC-2006-021, J. Phys. G: Nucl. Part. Phys. 34 995-1579

[28] Present Performance of the CERN Proton Linac, C.E. Hill, A.M. Lombardi, E. Tanke, M. Vretenar, Procs. of the 1998 Linear Accelerator Conf., Chicago, IL, p. 427 (1998)

[29] Past and present operation of the CERN PS Booster, K. Hanke, Int.J.Mod.Phys. A28 (2013) 1330019

[30] Fifty years of the CERN Proton Synchrotron, edited by S. Gilardoni and D. Manglunki, CERN-2011-004

[31] LHC Design Report Volume III, Part 2, Chapter 11-20, M. Benedikt, P. Collier, V. Mertens, J. Poole, K. Schindl, doi:10.5170/CERN-2004-003-V-3

[32] The LHC superconducting RF system, D. Boussard, T. P. R. Linnecar, LHCProject-Report-316, 1999.

[33] LHC Design Report Volume I, Part 2, Chapter 7, M. Benedikt, P. Collier, V. Mertens, J. Poole, K. Schindl, doi:10.5170/CERN-2004-003-V-3

[34] Level 1 Trigger Technical Design Report, the ATLAS Collaboration, CERN/LHCC/98-14 (1998)

[35] ATLAS High-Level Trigger, Data Acquisition and Controls, the ATLAS Collaboration, CERN-LHCC-2003-022

[36] The L1.5 Jet Trigger, the ATLAS Collaboration, Tamsett et al, ATL-COMDAQ-2012-015

[37] ATLAS Muon Spectrometer Technical Design Report, the ATLAS Collaboration, CERN/LHCC/9722, May 1997 
[38] ATLAS Liquid Argon Calorimeter Technical Design Report, the ATLAS Collaboration, CERN/LHCC/9641, December 1996

[39] ATLAS Tile Calorimeter Technical Design Report, the ATLAS Collaboration, CERN/LHCC/9642, December 1996

[40] ATLAS Inner Detector Technical Design Report, the ATLAS Collaboration, CERN/LHCC/97-16, ISBN 92-9083-102-2, 30 April 1997

[41] The ATLAS pixel detector, R. Klingenberg, Nuclear Instruments and Methods in Phys. Research A 579, Issue 2, 2007, Pages 664668, doi:10.1016/j.nima.2007.05.269

[42] ATLAS silicon microstrip detector system (SCT), Y. Unno, Nuclear Instruments and Methods in Phys. Research A 511 (2003) 5863

[43] The ATLAS TRT Barrel Detector, the ATLAS TRT collaboration, E Abat et al, JINST 3 P02014 2008

[44] Methods to quantify the performance of the primary vertex reconstruction in the ATLAS experiment under high luminosity conditions, Grimm et al, JPhys: Conference Series 396 (2012) 022041

[45] ALPGEN, a generator for hard multiparton processes in hadronic collisions, M. L. Mangano, F Piccinini, A. D. Polosa, M. Moretti, R. Pittau, JHEP 07 (2003)

[46] Measurement of the muon reconstruction performance of the ATLAS detector using 2011 and 2012 LHC protonproton collision data, the ATLAS Collaboration, Eur.Phys.J. C74 (2014) 3130

[47] Preliminary results on the muon reconstruction efficiency, momentum resolution, and momentum scale in ATLAS 2012 pp collision data, the ATLAS Collaboration, ATLAS-CONF-2013-088

[48] Performance of Missing Transverse Momentum Reconstruction in ATLAS studied in Proton-Proton Collisions recorded in 2012 at $\sqrt{s}=8 \mathrm{TeV}$, the ATLAS Collaboration, ATLAS-CONF-2013-082

[49] Readiness of the ATLAS Liquid Argon Calorimeter for LHC Collisions, The European Physical Journal C December 2010, Volume 70, Issue 3, pp 723-753

[50] Selection of jets produced in proton-proton collisions with the ATLAS detector using 2011 data, the ATLAS Collaboration, ATLAS-CONF-2012-020 
[51] Sudakov Form-factor in QCD, G.P. Korchemsky, Phys.Lett. B220 (1989) 629 JINR-E2-88-628

[52] Multiple Partonic Interactions in Herwig++, S. Gieseke, C. Rohr, arXiv:1302.4892v1

[53] Multiple Interactions and the Structure of Beam Remnants, T. Sjostrand and P. Z. Skands, JHEP 0403 (2004) 053

[54] An Eikonal model for multiparticle production in hadron hadron interactions, I. Borozan and M. H. Seymour, JHEP 0209 (2002) 015

[55] Measurement of the inclusive $\mathrm{W}+/$ - and $\mathrm{Z} /$ gamma* cross sections in the e and mu decay channels in pp collisions at $\sqrt{s}=7 \mathrm{TeV}$ with the ATLAS detector, the ATLAS collaboration, Phys. Rev. D 85072004

[56] A Measurement of WZ Production in Proton-Proton Collisions at $\sqrt{s}=$ $8 \mathrm{TeV}$ with the ATLAS Detector, the ATLAS Collaboration, ATLAS-CONF2013-021

[57] Event generation with SHERPA 1.1, T. Gleisberg, S. Hoeche, F. Krauss, M. Schonherr, S. Schumann, F. Siegert, J. Winter, JHEP 0902 (2009) 007

[58] Object Selections and Background estimates in the $h \rightarrow W W^{(*)} \rightarrow \ell \nu \ell \nu$ analysis with $20.7 \mathrm{fb}^{-1}$ of data collected with the ATLAS detector at $\sqrt{s}=$ 8TeV, the ATLAS ollaboration, ATL-COM-PHYS-2013-1504

[59] Fitting using finite Monte Carlo samples, R. Barlow and C. Beeston, Comp. Phys. Comm. 77 (1993) 219-228

[60] Kolmogorov-Smirnov Goodness-of-Fit Test http://www.itl.nist.gov/div898/handbook/eda/section3/eda35g.htm 


\section{SUMMARY}

The LHC physics program is now in full swing and has already turned heads in Stockholm. More discoveries are literally in the making, as the size of the recorded data set and the available energy range is ever increasing. So what is the LHC, and in specific ATLAS, looking for? Basically the entire accelerator and detector complex is one gigantic microscope. It searches for the smallest building blocks of nature, or elementary particles. Much like a Lego kit is build out of small elementary building blocks. Quite a few of those elementary particles are already discovered, like the familiar electrons and photons (light particles). Some examples of less familiar particles but similar in the fact that they are elementary are quarks, $\mathrm{W}$ and $\mathrm{Z}$ bosons, and the recently discovered Higgs boson among others. All these elementary particles interact with each other, bouncing off of each other and even destroying one another. A lot of these processes are already studied and documented in the Standard Model of particle physics, but not all.

Studying those processes is not as simple as just taking apart a Lego kit. To continue the analogy with Lego, protons are used as a fully build and functional Lego kit. However, physicists cannot simply dismantle a proton like a Lego house, and then study the building blocks. The only sensible thing they came up with was to accelerate the Lego houses to near the speed of light and then crash them into each other. This of course leaves an enormous mess of building block flying around. The same happens with the protons. The chaos of the collision is however not a total chaos. The angles and energies of the elementary particles coming out of the collision tells a lot about the nature of the particles itself, and 
about the processes in which they interact with other particles.

One previously unseen process that is about to be uncovered is $\mathrm{W}$ boson pair production in Double Parton Interactions. The process consists of two semiseparate productions of a $\mathrm{W}$ boson in a single proton-proton collision. Since this type of process requires the interaction of four initial particles, the dependence of these four parents on one another can be investigated in this case.

\section{Double Parton Interactions}

The process studied in this analysis is the Double Parton Interaction (DPI). A double parton interaction occurs when two protons collide, and two partons from each proton interact with two partons from the other proton. The theory behind this process is being studied for a few decades now, with a focus on the factorized hypothesis. The two processes in DPI are supposed to be only slightly dependent on each other, with only taking energy and flavor into account. Characteristics like spin and color charge are neglected.

On the experimental side there are already results measuring the total cross section (probability of occurrence) for DPI. Current measurements all have jets in the final state, meaning that the exact underlying process can not be accurately defined. The DPI process with highest cross section without jets is $\mathrm{W}$ boson pair production. When the $\mathrm{W}$ bosons decay leptonically all the interaction products are known and can be traced back to their origin, giving valuable information about the primary DPI process. DPI is also starting to be a more important background to searches for new physics. The cross section of DPI processes increase faster with increasing center of mass energy than processes in a single interaction, like new resonances. Since DPI can make an enormous variety of final states, mimicking all kinds of other processes, it is paramount to understand the physics behind it to distinguish it from physics beyond the Standard Model.

\section{W boson pair production in DPI}

The most abundant reconstructable objects in the detector by a large margin are jets. Jets are showers of constituent particles, which often can not be all fully resolved. This leaves the jet with a relatively smaller predictive power of the underlying process as an object that can be fully reconstructed. Double parton interactions with jets in the final state have therefore no known analytical power of the proton structure. In contrast to jets, $\mathrm{W}$ boson that decay into muons have the muon fully reconstructed with high precision. This muon has the same charge as the $\mathrm{W}$ boson, which gives valuable information about the flavor of the quarks in the proton that initiated the process. 
The $\mathrm{W}$ boson has another unique feature that is essential in its use as a proton structure probe. This quality is a quantum mechanical phenomenon and is related to the spin of the quarks in the proton, which can be related to a spinning top. The quarks always have spin, similar to the top if it wants to stay in balance. Now the remarkable feature of the $\mathrm{W}$ boson is that it can only see quarks with a left handed spin, and it is completely blind to quarks with their spin in the other direction. This selectiveness is propagated to the decay products of the $\mathrm{W}$ boson, and when two $\mathrm{W}$ bosons are produced in a single proton collision, the correlation between the two decay muons is a measure to the correlation of the initiating quarks.

\section{Analysis of W pair events with ATLAS}

Before the correlations between the final state muons can be connected to the proton structure, the existence of the $\mathrm{W}$ pair production by DPI process has to be proven to exist first. A random $\mathrm{W}$ pair can decay in a wide variety of ways, including jets, electrons, muons and tau particles with all four charge combinations. Most of these decay combinations, or final states, are very common for a large number of processes. There is one final state combination however, two muons with the same charge, that is very unique and has no known Standard Model processes producing it except $\mathrm{W}$ pairs produced in double parton interactions.

The promise of a unique final state should give a comfortable signal to noise ratio, however detection difficulty is playing a major role here. Event selection is based on the premise that there are two equal sign muons in the collision. A single muon production is extremely common, and sometimes a second muon comes into play from a secondary process. Also processes with three muons are quite common, of which the detector sometimes fails to see the third. Even four muons in the final state happens quite often where the detector misses two of them.

My analysis shows that background processes with one primary and one secondary (fake) muon, and three muons in the final state are dominating. The main point in the proof of the existence of the signal process, W pairs from DPI, is to correctly model these two background classes and quantify their individual contribution to the data set. I used loose event selection criteria to, on purpose, have a bad signal to noise ratio. This allowed me to treat the entire data set as consisting of background events (within error margins), in order to find the ratio in which these two backgrounds contributed.

After determining this ratio a set of Neural Networks is trained to distinguish background events from signal events. In this training a computer algorithm is instructed to look at differences between events produced by a background process and the DPI process under investigation. The result of this training is a 
black box that can give a probability to every new event being either from the background class it is trained for (one muon or three muon final state), or the event being produced by DPI.

In the third step data sets are constructed with simulated events, using the contributions for the background events found in the previous step to imitate the true data set without a signal contribution. Multiple simulated sets are constructed, while adding more and more simulated signal events. Finally these simulated sets are compared to the actual detector data set using the Neural Network outputs from step 2. In this way the simulated set that is most equal to the actual measured data set is determined, and since the number of signal events in that simulation is known, also the number of DPI events in the detector set can be estimated.

When the number of DPI events is known and significant, the actual DPI events can be analyzed. From theory we deduced that the direction of the two muons is connected to the way quarks are interacting with each other inside the proton. For this we have to count how many muons from DPI events are traversing the same half of the detector compared the the event count where the two muons fly through opposite sides of the detector as indicated in figure S1.
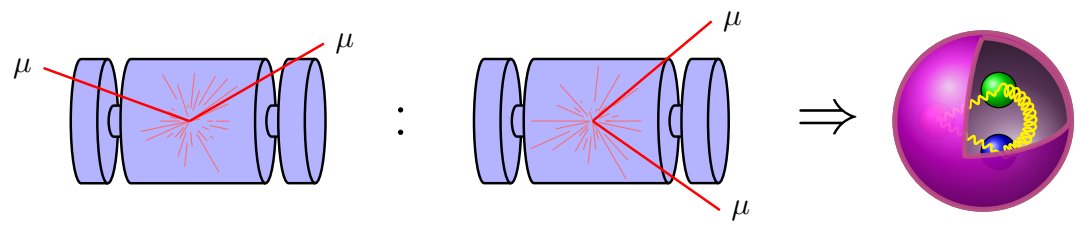

Figure S1: The ratio of DPI events where the final state muons traverse opposite hemispheres compared to events where they pass through the same hemisphere opens a window revealing the inner structure of the proton.

\section{Results from the W pair analysis}

In the analysis first the ratio of the two main background classes is determined: the one good muon plus one fake muon class and the three muon final state class. As mentioned before the event selection cuts are very loose so the full data set can be described by these two event classes within error margin. All the important variables like muon energy and direction, and the properties of the neutrino that is also produced when the $\mathrm{W}$ boson decays, are drawn in histograms. Each event class has its own histograms for all variables from simulation, as does the detector data set. By varying the normalizations the simulated histograms are fitted to 
the data histograms, after which the relative contributions in the data can be extracted from this fit result.

The result of this fit tells us that the contributions of the fake muon background compared to the three muon background has a ratio of 1:2. This means that the data set obtained with the loose cuts consists of 1 part fake muon events, and 2 parts three muon final state events. The signal contribution is in the error margins of this ratio. The result of the fitting procedure on the muon energy is shown in figure $\mathrm{S} 2$.
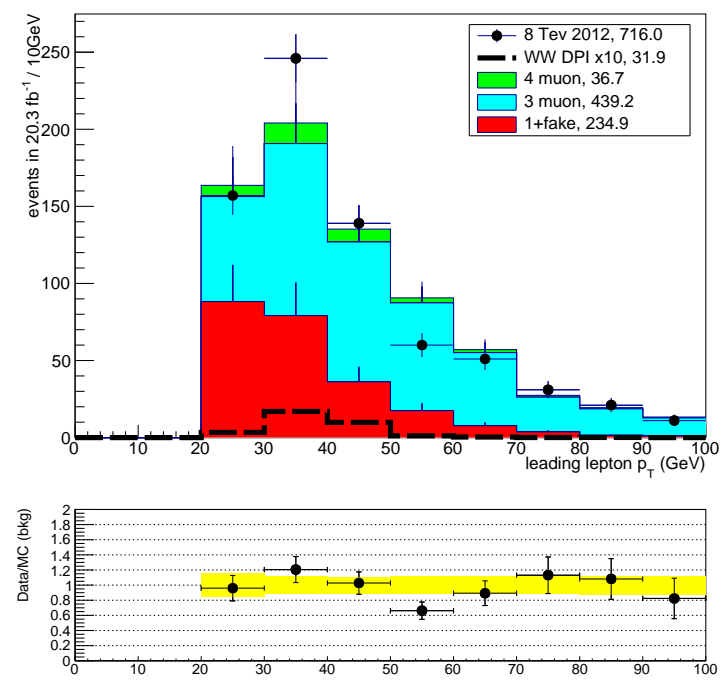

Figure S2: Muon momentum fit results. The red area is the fake muon class contribution, and the blue area the three muon final state contribution. They are responsible for the bulk of the events. The four muon final state is added in green, and is smaller than the error on the main two event classes. The same is true for the DPI signal events indicated with the dashed line.

With this result the pseudosets are created, with increasing signal contributions. As a step prove the existence of the $\mathrm{W}$ pair production process in DPI events, the pseudoset without any signal events is compared to the detector data using the Neural Network responses. This procedure is called a null hypothesis test. When the null hypothesis can be rejected, the detector data is considerably different from the background only hypothesis. This means that there is another process contributing significantly. Unfortunately the null hypothesis in this anal- 
ysis is a valid one, meaning the pseudoset without signal events is similar to the detector data within error margins.

However, not all is lost. The Neural Network responses are linear and show good stability with increasing signal event contributions, as shown in figure S3. This allows for the identification of the most likely signal event count in the detector data. For this we only have to compare the analysis result from the detector

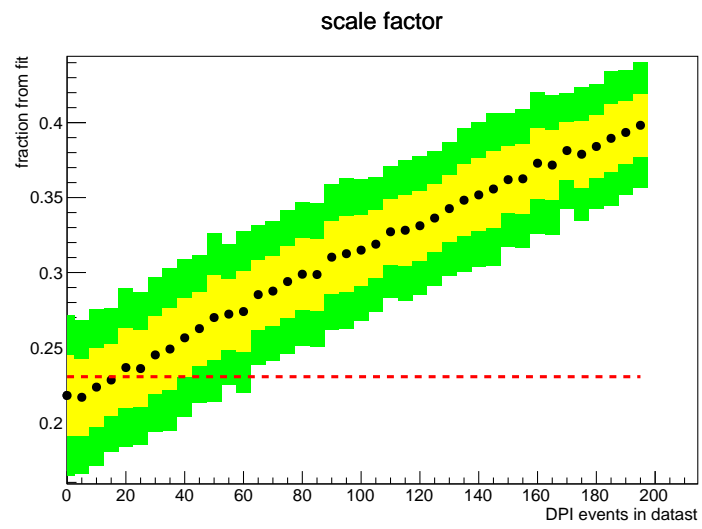

Figure S3: Pseudoset ensemble DPI fractions. The yellow band shows the standard deviation in the fraction found for pseudosets with the same number of signal events added, the green band is the $95 \%$ CL. The red dashed line indicates the value found for the detector data set.

data set, indicated with a red dashed line, to the pseudosets with simulated signal events. The figure shows that the most likely signal event count in the data is 15 events.

With the null hypothesis still valid and the maximum likelihood of only 15 signal events in a set of 750 events total, studying the structure of the proton is not possible yet. This is because the ratio shown in figure S1 will only measure this quantity in the background events. This measurement is only valid in the signal events, and will therefore not have a valid physics interpretation unfortunately.

\section{Conclusion and outlook}

The results of the analysis show that the $\mathrm{W}$ boson pair production in DPI events is still an elusive process. The main contamination of the signal comes from the difficulty to properly reconstruct events with a different signature. The two main background processes are identified, and should be the main focus of future 
research into this process. When more data is acquired the cuts can be tightened, improving the signal to noise ratio. When the noise level is low enough real physics can be done to unravel more of the proton structure mysteries. The amount of data used in this analysis is recorded over the course of about a year. The ATLAS detector is planned to run for at least a couple of decades at increasing event rates, so the problem of too little signal events should be solved in the coming years. This gives positive prospects for the physics potential of the $\mathrm{W}$ boson pair production in DPI events. 


\section{SAMENVATTING}

Het natuurkundeprogramma van de LHC draait op volle kracht en heeft een cruciale rol gespeeld in de Nobelprijs voor Natuurkunde 2012. Met het vergroten van het aantal metingen en het verhogen van de botsingsenergie zitten verdere ontdekkingen letterlijk in de pijplijn. Waar is de LHC, en met name ATLAS, naar op zoek? Kort gezegd is het gehele versnellercomplex inclusief detectoren niet meer dan een gigantische microscoop. Het speurt naar de allerkleinste bouwstenen van de natuur, de elementaire deeltjes. Net als een Lego bouwpakket is opgebouwd uit kleine stukjes. Veel van deze elementaire deeltjes zijn al ontdekt, bijvoorbeeld de welbekende elektronen en fotonen (lichtdeeltje). Een aantal voorbeelden van minder bekende deeltjes zijn onder andere de quarks, $\mathrm{W}$ en $\mathrm{Z}$ bosonen en het recent ontdekte Higgs boson. Al deze elementaire deeltjes hebben interactie met elkaar, trekken elkaar aan en stoten elkaar af, of vernietigen elkaar. Een groot aantal van deze processen zijn al bestudeerd en opgenomen in het Standaard Model van de deeltjesfysica, maar dit is nog niet compleet.

Het bestuderen van deze processen is niet zo eenvoudig als het simpelweg uit elkaar halen van een Lego bouwpakket. In de analogie met Lego is het proton een volledig opgebouwd Lego pakket. Natuurkundigen kunnen helaas niet het proton stukje voor stukje uit elkaar halen als een Lego huis, om vervolgens de bouwstenen te bestuderen. De enige oplossing die ze hebben kunnen bedenken is het versnellen van het Lego huis tot bijna de lichtsnelheid, om ze vervolgens compleet te vernietigen door ze met deze snelheid tegen elkaar te laten botsen. Dit veroorzaakt natuurlijk een enorme puinhoop van rondvliegende bouwstenen. Hetzelfde principe geld voor protonen. De chaos van de botsing is echter geen 
complete chaos. De richting en energie van de elementaire deeltjes uit de botsing vertellen veel informatie over het deeltje zelf, en de processen waarmee ze met andere deeltjes reageren.

Een specifiek proces dat tot op heden nog niet is waargenomen, maar waar de LHC en ATLAS wel gevoelig voor zijn, is W boson paar productie in Double Parton Interactions. Dit proces bestaat uit twee semi-afhankelijke productieprocessen van een $\mathrm{W}$ boson in een enkele proton-proton botsing. Omdat dit proces de interactie van aanvankelijk vier deeltjes vereist, kan hiermee de afhankelijkheidsrelaties in dit systeem van vier deeltjes bestudeerd worden.

\section{Double Parton Interactions}

In mijn analyse bestudeer ik de Double Parton Interaction (DPI). Het DPI proces vind plaats wanneer twee protonen met elkaar botsen, waarbij twee partonen van het ene proton een interactie aangaan met twee partonen van het andere proton. De theorie die dit proces beschrijft word al langere tijd bestudeerd, met de focus op de gefactoriseerde hypothese waarbij de partonen in eerste instantie onafhankelijk zijn van elkaar. De twee interacties zijn onder deze hypothese slechts licht afhankelijk van elkaar, alleen de afzonderlijke energie en het type quark worden meegenomen in de berekening. Attributen als spin en kleur worden genegeerd.

Aan de experimentele kant zijn er al enkele resultaten die de werkzame doorsnede (kans van optreden) van DPI bepalen. Deze metingen zijn allen gedaan aan processen met jets in de eindtoestand, wat betekent dat de onderliggende processen niet exact achterhaald kunnen worden. Het DPI proces met de hoogste werkzame doorsnede zonder jets in de eindtoestand is $\mathrm{W}$ boson paar productie. Wanneer beide $\mathrm{W}$ bosonen vervallen in leptonen zijn alle botsingsproducten meetbaar en kunnen deze herleid worden naar het interactiepunt. Dit geeft waardevolle informatie over het DPI proces zelf. DPI wordt in de nieuwe generatie experimenten ook een belangrijk achtergrondproces bij de zoektocht naar nieuwe fysica. De werkzame doorsnede van DPI processen groeit harder bij hogere botsingsenergie dan die van een enkele interactie, zoals bij nieuw te ontdekken deeltjes. Aangezien DPI een enorm aantal verschillende eindtoestanden kan produceren, kan het heel veel lijken op bijna elk mogelijk proces binnen en buiten het Standaard Model. Daarom is het van cruciaal belang de onderliggende fysica van dit proces te begrijpen om het te kunnen onderscheiden van nieuwe fysica. 


\section{W boson paar productie in DPI}

Veruit de meeste objecten die in de detector gereconstrueerd kunnen worden zijn jets. Jets zijn lawines van deeltjes, waarvan de individuele onderdelen vaak niet helemaal gereconstrueerd kunnen. Hierdoor geeft een jet minder informatie over het onderliggende proces dan objecten (deeltjes) die volledig gereconstrueerd kunnen worden. Botsingen met een DPI proces waarin jets geproduceerd worden geven daarom geen analytische informatie over de interne structuur van het proton. In tegenstelling tot $\mathrm{W}$ bosonen die vervallen naar jets, kunnen $\mathrm{W}$ bosonen die vervallen naar muonen volledig en met hoge precisie gereconstrueerd worden. Dit muon heeft dezelfde electrische lading als het $\mathrm{W}$ boson in het proces, en geeft daarmee waardevolle informatie over het quark in het proton dat aan het begin van het proces stond.

Het $\mathrm{W}$ boson heeft een unieke eigenschap welke essentieel is voor haar toepassing in onderzoek naar de structuur van het proton. Deze kwantummechanische eigenschap is gerelateerd aan de spin van de quarks in het proton, welke te vergelijken valt met de rotatie van een draaitol. Quarks hebben altijd spin, evenals de draaitol moet roteren om in balans te blijven. $\mathrm{Nu}$ heeft het $\mathrm{W}$ boson het eigenaardige kenmerk dat het alleen quarks kan zien die linksom draaien, voor de quarks met rechtshandige spin is het compleet blind. Deze selectiviteit wordt doorgegeven aan de vervalsproducten van het $\mathrm{W}$ boson. Wanneer twee $\mathrm{W}$ bosonen worden geproduceerd in een enkele proton-proton botsing, heeft de correlatie tussen de twee uitgaande muonen een directe verbinding met de correlatie tussen de quarks in de botsing.

\section{W boson paar analyse met ATLAS}

Voor de correlatie tussen de uitgaande muonen gerelateerd kan worden met de protonstructuur, is het noodzakelijk eerst aan te tonen dat DPI in staat is W paren te produceren. Een willekeurig W paar kan in een groot aantal combinaties van eindtoestanden vervallen, bestaande uit jets, elektronen, muonen en tau leptonen. Veel van deze combinaties van vervalsproducten deelt DPI met een groot aantal andere Standaard Model processen, wat betekent dat het voor deze eindtoestanden bijzonder lastig is te achterhalen welk specifiek proces aan de basis stond. Een van deze combinaties echter is uniek voor W paar productie in DPI, en dat is de productie van twee muonen met gelijke lading.

Een unieke eindtoestand zou een comfortabele signaal-ruisverhouding moeten geven, echter detectie inefficiëntie speelt hier een belangrijke rol. Event selectie is gebaseerd op het feit dat er twee muonen met gelijke lading in de eindtoestand aanwezig zijn. De productie van een enkele muon komt bijzonder vaak voor, en soms word er bij een botsing een tweede muon geproduceerd in een secundair 
proces. Ook zijn er processen binnen het Standaard Model waarbij drie muonen geproduceerd worden, waarvan de detector er soms eentje mist. Zelfs vier muonen in de eindtoestand komt relatief vaak voor, waarbij het mogelijk is dat de detector twee hiervan niet ziet.

Mijn analyse toont aan dat processen met een muon uit een primair proces en een tweede uit een secondair proces, en processen met drie muonen dominant zijn in de gefilterde dataset. Voor het bewijzen van het bestaan van het signaalproces is het van cruciaal belang dat deze twee achtergrondcategorieën correct zijn gemodelleerd. Alleen met een correct model is het mogelijk de individuele contributie aan de detector dataset te bepalen. Voor het creëren van de dataset heb ik gekozen voor ruime selectiecriteria om juist een lage signaal-ruisverhouding te krijgen. Hiermee kan ik de gehele dataset beschouwen als bestaande uit achtergrondprocessen (binnen de foutenmarge), om zo de ratio van contributie voor de twee categorieën te bepalen.

Naast het bepalen van deze ratio van de achtergrondprocessen is een tweetal Neurale Netwerken getraind op het onderscheiden van signaal en achtergrond. Gedurende deze training leert het netwerk onderscheid te maken tussen een specifieke achtergrondcategorie en het DPI proces door te kijken naar verschillen in variabelen als energie en uitgaande hoek van de deeltjes. Het resultaat van de training is een algoritme dat de waarschijnlijkheid kan geven aan een event of het behoort tot de achtergrondcategorie waar het netwerk op getraind is, of dat het event geproduceerd is door DPI.

De volgende stap is het creëren van datasets met gesimuleerde events. Hierbij wordt gebruik gemaakt van de relatieve contributies van de achtergrondprocessen gevonden in de vorige stap. Op deze wijze wordt een dataset gesimuleerd zonder enige signaalcontributie. Vervolgens worden meerdere sets gecreërd, elk met meer gesimuleerde DPI events. Tot slot worden deze gesimuleerde datasets vergeleken met de detector dataset gebruik makende van de getrainde Neurale Netwerken. Hiermee wordt de gesimuleerde set bepaald die het meest overeenkomt met de detector dataset. Sinds het aantal signaalevents in deze set bekend is kan hiermee het aantal DPI events in de detector dataset bepaald worden.

Wanneer het aantal DPI events bekend is en significant groot, kunnen deze geanalyseerd worden. In theorie is de richting van de twee uitgaande muonen gecorreleerd aan de quarks in het proton welke de interactie aangaan. De grootte van de correlatie staat in directe relatie met de ratio van het aantal muonparen dat door dezelfde hemisfeer van de detector reist, en het aantal muonparen dat de tegenoverstaande hemisferen doorkruist. Dit is schematisch weergegeven in figuur S1. 

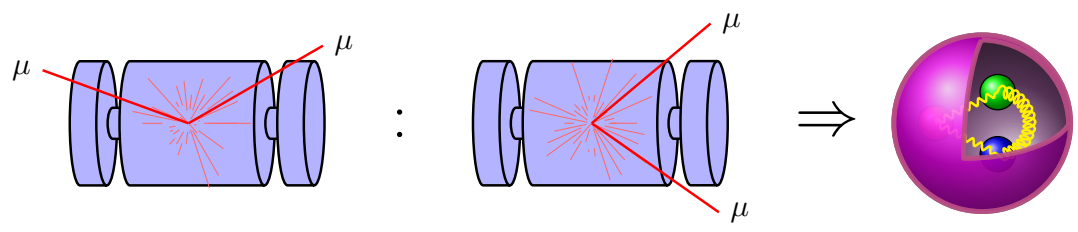

Figuur S1: De ratio van DPI events waarbij de muonen door dezelfde hemisfeer reizen vergeleken met het aantal events waarbij de muonen door de tegenovergestelde hemisfeer gaan opent een nieuw blikveld op de interne structuur van het proton.

\section{Resultaten van de $\mathrm{W}$ boson paar analyse}

De eerste stap in de analyse is het bepalen van de ratio tussen de twee dominante achtergrondcategorieën: de events met het tweede muon uit een secondair proces, en de processen met drie muonen in de eindtoestand. Zoals al eerder aangegeven zijn de selectie criteria dusdanig ruim gekozen zodat de gehele dataset, binnen de foutenmarge, kan worden beschouwd als bestaande uit alleen deze twee procescategorieën. Alle relevante variabelen in de events, zoals de muon energie en uitgaande hoek, en de eigenschappen van het neutrino in het $\mathrm{W}$ boson verval, zijn gespecificeerd in histogrammen. Elke procescategorie heeft zijn eigen set histogrammen voor elke variabele in het event, evenals de detectordataset. Door het varieren van de normalisatie van de gesimuleerde histogrammen kunnen deze passend gemaakt worden aan de detectordataset. Na deze afstellingsprocedure kan de relatieve contributie van de twee dominante categorieën afgelezen worden uit de normalisatieconstantes.

Deze waarden geven een ratio van 1:2. Dit betekend dat de gemeten dataset met de ruime selectiecriteria beschouwd kan worden als bestaande uit 1 deel events met een secondair muon, en 2 delen events met drie muonen in de eindtoestand. De contributie van het DPI signaalproces bevindt zich in de foutenmarge van deze ratio. Als voorbeeld van deze afstellingsprocedure is het histogram van de muonenergie weergegeven in figuur S2.

$\mathrm{Nu}$ de relatieve contributies bepaald zijn kunnen de pseudosets gecreëerd worden. Deze datasets worden gevuld met events uit de achtergrondcategorieën in de juiste verhoudingen, en het DPI signaal. Het verschil tussen de pseudosets is de contributie van het signaalproces. Onderdeel van het bewijs dat er $\mathrm{W}$ paren uit het DPI proces aanwezig zijn in de detectordataset is de null hypothese test. In deze test worden pseudosets zonder DPI signaal contributie vergeleken met de detectordataset door middel van de Neurale Netwerk modellen. Wanneer de null hypothese verworpen kan worden doordat pseudosets sterk afwijken van de detec- 

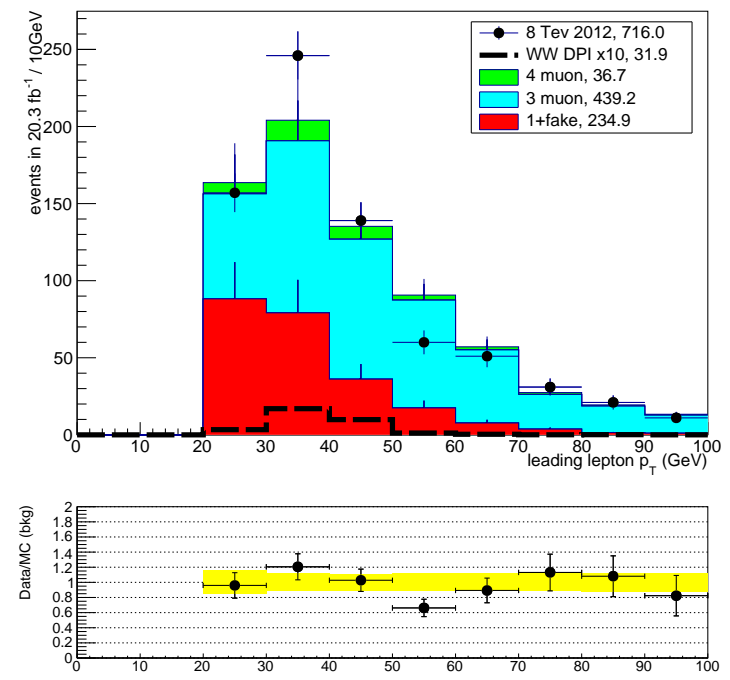

Figuur S2: Histogram van de energie van het muon na de afstellingsprocedure. Het rode segment geeft de contributie van de events met een secondair muon, het blauwe segment de events met drie muonen. Deze twee typen zijn veruit dominant in de dataset. De events met vier muonen zijn weergegeven in groen. Deze contributie is kleiner dan de foutenmarge op de twee dominante processen. Dit geldt ook voor de contributie van het DPI signaal weergegeven met een onderbroken lijn.

tordataset, betekend dat er een extra proces significant bijdraagt. In mijn analyse kan de null hypothese echter nog niet verworpen worden omdat de pseudosets te sterk overeenkomen met de detectordataset.

Vervolgens kunnen de signaalhypotheses gebruikt worden om een schatting te maken hoeveel $\mathrm{W}$ boson paren geproduceerd door DPI in de detectordataset zitten. De respons van de Neurale Netwerken vertoont lineaire stabiliteit zoals weergegeven in figuur S3. Hiervoor vergelijken we de analyseresultaten van de detectordataset, aangegeven met een rode onderbroken lijn, met de analyse van de pseudosets met gesimuleerde events. De figuur geeft weer dat de pseudosets met 15 signaalevents de grootste gelijkenis vertoont met de detectordataset.

Met de null hypothese nog steeds geldig en een maximale waarschijnlijkheid van 15 signaalevents in een dataset van 750 events is het nog niet mogelijk de proton structuur te analyseren met deze methode. Dit komt doordat de ratio in 


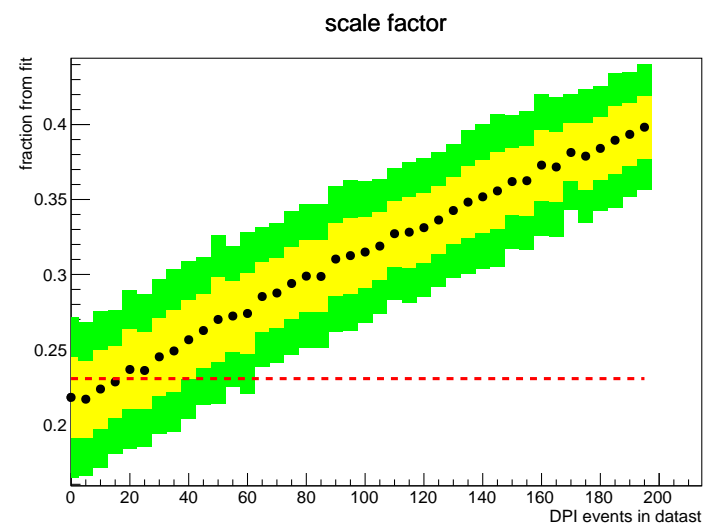

Figuur S3: Pseudoset ensemble relatieve DPI contributie. De gele band geeft de standaard deviatie weer op de gevonden fractie signaalevents in pseudosets met gelijk aantal DPI events toegevoegd. De groene band is de 95\% CL. De rode onderbroken lijn geeft de gevonden fractie signaalevents in de detectordataset.

figuur S1 in deze dataset gemeten zal worden voor de achtergrondprocessen. Deze meting is alleen geschikt voor W paren geproduceerd in DPI, en zal daardoor in deze dataset geen natuurkundige waarde hebben.

\section{Conclusie en perspectief}

De resultaten van de analyse geven aan dat W boson paar productie in DPI nog steeds buiten experimenteel bereik ligt. De dominante achtergrond op het signaal wordt veroorzaakt doordat processen met een andere eindtoestand onvolledig gereconstrueerd worden, en vervolgens op het signaal gaan lijken. De twee achtergrondcategorien̈ die het grootste effect hebben zijn geidentificeerd en zouden de focus van het vervolgonderzoek moeten zijn. Wanneer er meer statistiek aan de detectordataset toegevoegd wordt, kunnnen de selectiecriteria strenger gekozen worden. Dit zal de signaal-ruisverhouding aanzienlijk verbeteren. Als deze verhouding eenmaal laag genoeg is kan het DPI proces worden gebruikt om onderzoek naar de structuur van het proton te doen. De dataset in deze analyse is opgebouwd gedurende ongeveer een jaar. De planning voor de ATLAS detector gaat enkele tientallen jaren ver, wat het probleem van de lage statistiek ongetwijfeld gaat oplossen. Dit geeft een positief perspectief voor de natuurkundige waarde van de $\mathrm{W}$ boson paar productie in DPI events. 


\section{ACKNOWLEDGEMENTS}

Last decade not a single day has gone by that Particle Physics did not cross my mind in one way or another. The single person to blame for that is my promotor, Bob van Eijk. He introduced me 10 years ago to the wonderful world of the Particle Zoo (a term I rarely hear nowadays), and pointed me to all the exciting possibilities that working in physics had to offer. But most importantly, he let me choose my own path and first figure it out myself. This freedom allowed me to take the turns and decisions that made this research possible.

Two people have been awesome during my time at Nikhef. Particle Physics may be a nice career choice, but working with Pieter van der Deijl and Hartger Weits made it an extraordinary one. I will remember the endless lunch discussions and particular world both of you have for a long time, and I look forward to a redo every now and then.

Special thanks go to my parents and brother; Mama, Papa, Niels, zonder jullie was ik nooit zover gekomen. Jullie oneindige geduld, steun en interesse in deze ver van mijn bed show was onmisbaar. Ik hou ontzettend veel van jullie.

And most importantly I'd like to thank my wife Jelena and my daughters Milena \& Kiki, you reminded me every day why I was doing this. Your boundless trust and love gave me the energy to pull this off. You are the sunshine in my life, this would not have happened if it weren't for you. 